\title{
Catalytic Water Cleaning: Materials and Transport Aspects
}
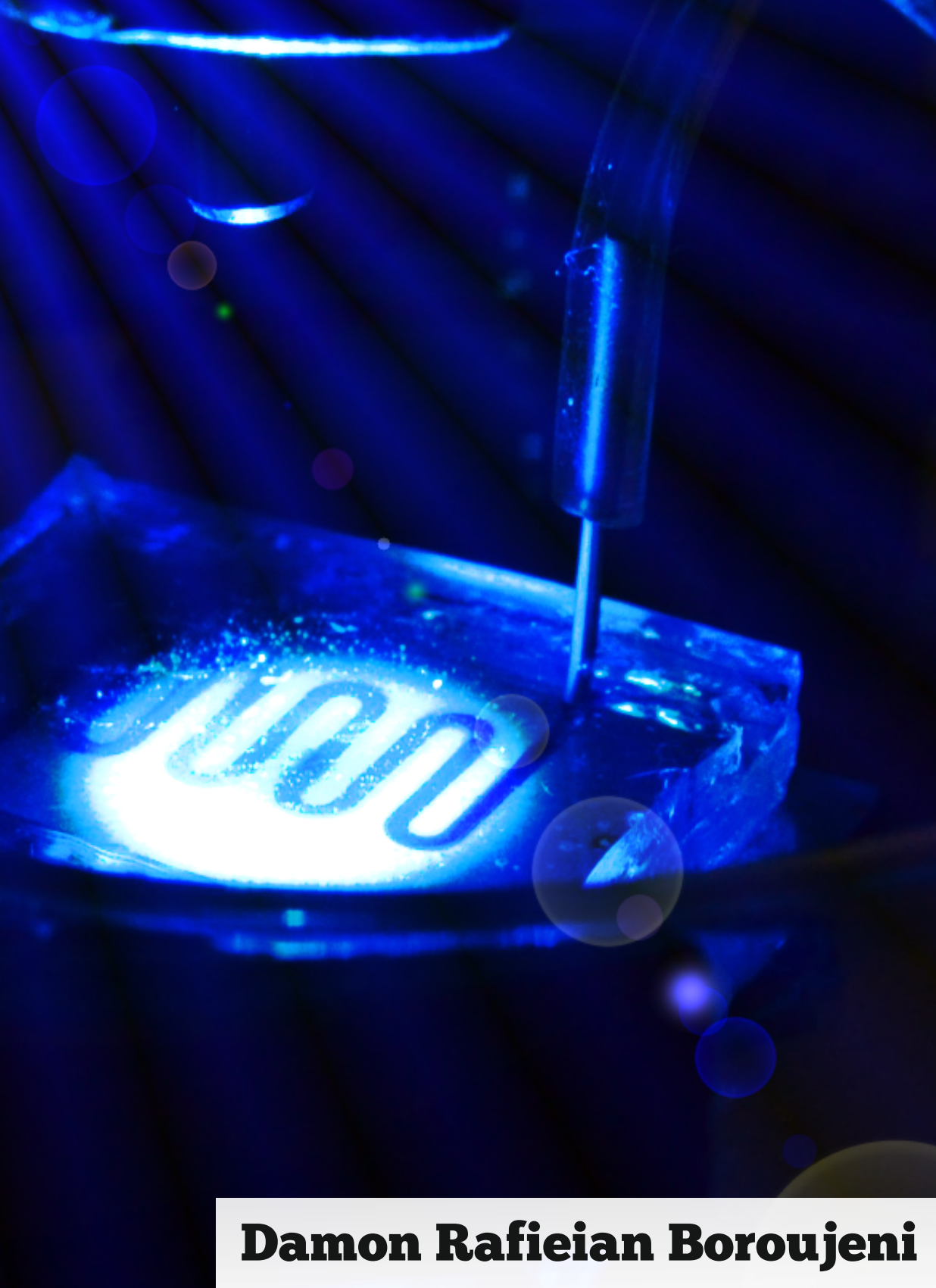
CATALYTIC WATER CLEANING:

MATERIALS AND TRANSPORT

ASPECTS 
Promotion committee

Promotor

Prof. dr. ir. R.G.H Lammertink

Other members

Prof. dr. ir. Leon Lefferts

Prof. dr. Guido Mul

Prof. dr. Han Gardeniers

Prof. dr. ir. Kristof Demeestere

Dr. ir. Tom J. Savenije

This thesis is part of NanoNextNL, a micro and nanotechnology innovation consortium of the Government of the Netherlands and 130 partners from academia and industry. More information on www.nanonextnl.nl.

It was carried out at the

Soft matter Fluidics and Interfaces (SFI) group,

Department of Science and Technology

and MESA+ Institute for Nanotechnology,

University of Twente, P.O. Box 217,

7500 AE Enschede, The Netherlands.

Cover design: Photograph of a PDMS based microreactor.

Design by Damon Rafieian

ISBN: 978-90-365-4078-0

DOI: $10.3990 / 1.9789036540780$

URL: http://dx.doi.org/10.3990/1.9789036540780

Printed by Gilderprint, Enschede, The Netherlands 


\section{CATALYTIC WATER CLEANING: MATERIALS AND TRANSPORT ASPECTS}

\section{DISSERTATION}

to obtain

the degree of doctor at the University of Twente,

on the authority of the rector magnificus,

prof. dr. H. Brinksma,

on account of the decision of the graduation committee,

to be publicly defended on

on Friday the $11^{\text {th }}$ of March, 2016 at 12:45

by

Damon Rafieian Boroujeni

born on $20^{\text {th }}$ of September, 1982

in Tehran, Iran 
This thesis has been approved by:

Prof. dr. ir. R.G.H Lammertink 
In memory of my grandfather.

You will never be forgotten. 



\section{Contents}

1 Introduction $\quad 11$

1.1 Heterogeneous Photocatalysis . . . . . . . . . . . . . . 14

1.1.1 Titanium Dioxide. . . . . . . . . . . 16

1.1.2 Photocatalytic reactors . . . . . . . . . . . . . 19

1.1.3 Kinetics of photocatalytic reactions . . . . . . . . . . 22

1.2 Hydrogenation ....................... 24

1.2.1 Membrane reactors . . . . . . . . . . . 25

1.2.2 Carbon nanofiber based catalyst support . . . . . . . . 26

1.3 Thesis outline . . . . . . . . . . . . . . . . . . . . . . 28

2 Selective deposition of anatase and rutile $\mathrm{TiO}_{2} \quad 41$

2.1 Introduction . . . . . . . . . . . . . . . . . 42

2.2 Experimental . . . . . . . . . . . . . . . . . . . . 43

2.2.1 Deposition of $\mathrm{TiO}_{2}$ thin film . . . . . . . . . . . . 43

2.2.2 $\mathrm{TiO}_{2}$ thin film characterization . . . . . . . . . . 43

2.3 Results and Discussion . . . . . . . . . . . . . . . . . . . 44

2.3.1 $\mathrm{TiO}_{2}$ Thin film deposition . . . . . . . . . . . . 44

2.3.2 Optical properties ................ . . 44

2.3.3 Structure and chemistry . . . . . . . . . . . 46

2.3.4 Charge carriers mobility . . . . . . . . . . . 48

2.4 Conclusion ....................... 50

3 Intrinsic Photocatalytic Assessment of Reactively Sputtered $\mathrm{TiO}_{2}$ Films 59

3.1 Introduction . . . . . . . . . . . . . . . . . . . 60

3.2 Materials and methods . . . . . . . . . . . . . . 61

3.2.1 Photocatalyst Synthesis . . . . . . . . . . . 61

3.2.2 Catalyst layer characterization . . . . . . . . . . 62 
3.2.3 Microreactor fabrication . . . . . . . . . . . 62

3.2.4 Microreactor operation . . . . . . . . . . . . . . 62

3.3 Model . . . . . . . . . . . . . . . . . . . 63

3.4 Results and Discussion . . . . . . . . . . . . . . . . . . 66

3.4.1 Film deposition and characterization . . . . . . . . 66

3.4.2 Photocatalytic performance . . . . . . . . . . 67

3.4.3 Effect of photocatalyst thickness . . . . . . . . . 71

3.5 Conclusion . . . . . . . . . . . . . . . . 72

4 In-situ AFM study of $\mathrm{Si} / \mathrm{TiO}_{2}$ heterojunctions $\quad 77$

4.1 Introduction . . . . . . . . . . . . . . . . . . . . 78

4.2 Experimental . . . . . . . . . . . . . . . . 80

4.2.1 Patterned $\mathrm{TiO}_{2}$ thin film preparation . . . . . . . . . 80

4.2.2 Atomic force microscopy . . . . . . . . . . . . . 81

4.2.3 Photocatalytic measurement . . . . . . . . . . . . 82

4.3 Results and Discussion . . . . . . . . . . . . . . . . . . . . . 84

4.3 .1 AFM .................... 84

4.3.2 Photocatalytic assessment . . . . . . . . . . 87

4.4 Conclusion ........................ 90

5 Porous Titanium Dioxide Thin Film; Experimental and Modeling Study 95

5.1 Introduction . . . . . . . . . . . . . . . . . . 96

5.2 Experimental . . . . . . . . . . . . . . . . . . . . . . 98

5.2.1 $\mathrm{TiO}_{2}$ immobilization . . . . . . . . . . . . . . . 98

5.2 .2 Microreactor fabrication . . . . . . . . . . . . . . 98

5.2 .3 Catalyst layer characterization . . . . . . . . . . . . 99

5.2.4 Microreactor operation . . . . . . . . . . . 100

5.3 Model . . . . . . . . . . . . . . . . . . . 101

5.3.1 Light independent model . . . . . . . . . . . . . . 101

5.3.2 Light dependent model . . . . . . . . . . . . . . . . 103

5.4 Results and Discussion . . . . . . . . . . . . . . . . . . . . . . 104

5.4.1 Film preparation ................. 104

5.4 .2 Light independent model (LIM) . . . . . . . . . . . 107

5.4.3 Light dependent model $(\mathrm{LDM}) \ldots 109$ 
5.4.4 Criteria for neglecting light intensity . . . . . . . . . . . 111

5.4.5 Updated performance parameters . . . . . . . . . . . 112

5.5 Conclusions . . . . . . . . . . . . . . . . . . . . . 114

6 Hydrogenation of nitrite in a membrane microreactor 121

6.1 Introduction . . . . . . . . . . . . . . . . . . . . . . . 122

6.2 Experimental . . . . . . . . . . . . . . . . . 124

6.3 Catalytic assessment . . . . . . . . . . . . . . 126

6.3.1 $\mathrm{H}_{2}$ outside reactor . . . . . . . . . . . . 127

6.3.2 $\mathrm{H}_{2}$ inside reactor . . . . . . . . . . . . . 128

6.3.3 Packed bed reactor . . . . . . . . . . . . . . . . . 128

6.4 Characterization . . . . . . . . . . . . . . . . 129

6.5 Results and Discussion . . . . . . . . . . . . . . . . . 129

6.5.1 Nickel deposition . . . . . . . . . . . . . . . . . 129

6.5.2 Reduction temperature ............... 130

6.5.3 CNF growth temperature ............... . . 131

6.5.4 PDMS coating ...................... 135

6.5.5 Catalytic nitrite hydrogenation . . . . . . . . . 136

6.6 Conclusion . . . . . . . . . . . . . . . . . . 139

7 Summary and Outlook $\quad 145$

7.1 Summary . . . . . . . . . . . . . . . . . . 145

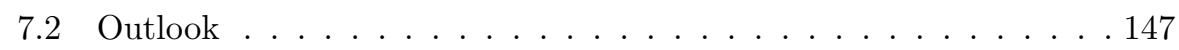

7.2.1 Scaling up; Design and fabrication of a disk reactor . . . . . 147

7.2.2 Catalytic hydrogenation of nitrate combined with photocatalysis . . . . . . . . . . . . . . . . . 149

$\begin{array}{ll}\text { Algemene Nederlandse samenvatting } & 153\end{array}$

$\begin{array}{ll}\text { Acknowledgements } & 157\end{array}$ 



\section{CHAPTER 1}

\section{Introduction}

The first part of this chapter covers a general overview on photocatalysis and photocatalytic reactors. The advantages of photocatalysis over other advanced oxidation processes (AOP) with focus on water purification will be discussed. The second part presents an overview on denitrification in membrane microreactors. The advantageous role of carbon nanofibers as catalyst support in these reactors will be discussed as well. 
According to the United Nations, unsanitary water takes more life worldwide than war. The fresh water constitutes a tiny fraction of the whole earth water supply while water consumption has increased almost twice as much as the population growth [1]. It is estimated that by 20303.9 billion people will face water scarcity [2]. Worse still, according to World Health Organization 1.1 billion people do not have access to potable water and 2.6 billion live without proper sanitation [3]. This threatens 2.2 million people with diarrheal oriented diseases on a yearly basis and tragically most of them are children younger than 5 years old [4]. This calls for seeking new methods for water purification since conventional water purification methods do not always satisfy new drinking water standards. In principle water treatment methods are categorized into phase separation (adsorption processes, stripping techniques) and methods which eliminate the contaminants (chemical oxidation/reduction) [5]. Among the latter one advanced oxidation processes (AOP) gained lots of attention recently for the removal of micro-pollutants, pathogenic bacteria and viruses [6]. By definition AOPs are (near ambient temperature and pressure) water treatment processes which involve the generation of hydroxyl radicals in sufficient quantity for water purification [7]. In almost all of AOPs the prominent working horse for oxidation is hydroxyl radicals $\left(\mathrm{OH}^{*}\right)$. They are able to oxidatively destroy a wide range of organic contaminants in water. Below some of the established AOPs are briefly explained.

\section{$\mathrm{H}_{2} \mathrm{O}_{2} / \mathrm{O}_{3}$}

The hydroxyl radicals are generated through reaction of ozone and hydrogen peroxide into water. One of the main disadvantage of this method is the formation of carcinogenic bromate in case of bromide-containing waters [8]. In addition, the excess of $\mathrm{H}_{2} \mathrm{O}_{2}$ should be treated after the reaction.

\section{$\mathrm{H}_{2} \mathrm{O}_{2} / \mathrm{UV}$}

The process is based on the photolysis of aqueous $\mathrm{H}_{2} \mathrm{O}_{2}$ by using light with lower than $280 \mathrm{~nm}$ (UV-C) wavelength, cleaving the O-O bond and forming $\mathrm{OH}^{*}$. Although in this method there is no potential for bromate formation, the removal of $\mathrm{OH}^{\cdot}$ remains an issue [9].

\section{Fenton's Reaction}

In this method $\mathrm{OH}^{\cdot}$ radicals are produced by Fenton reagent [10] and added 
$\mathrm{H}_{2} \mathrm{O}_{2}$ and salts. No special apparatus and reactants are needed for the formation of the $\mathrm{OH}^{*}$ radicals. In order to sustain iron in solution, the $\mathrm{pH}$ should be kept less than 2.5, which increases operation and maintenance cost.

\section{Photolysis}

In this method $\mathrm{OH}^{*}$ radicals are generated through vacuum ultraviolet (VUV $<195 \mathrm{~nm}$ ) irradiation. The significant strong point of this method is that reduced additional chemicals are required for this process. The cost and transparency at very low wavelength makes the process less appealing. In addition due to high absorption coefficients of water and organic pollutants at this wavelength the efficiency is noticeably decreased.

\section{Photocatalysis}

In contrast to the aforementioned AOPs, photocatalysis requires no additional chemicals for purification. Generally no toxic intermediates are formed during photcatalytic reactions. Heterogeneous photocatalysis recently attracts attention for elimination of a subclass of organic contaminants; endocrine disruptor chemicals (EDC) [11] and pharmaceutical contaminants. Conventional methods are mostly utilized for removing suspended solids and biodegradable organic compounds in water and they are not efficient for these low concentration synthetic pollutants $[12,13]$.

Photocatalysis can be utilized to break down a wide variety of organic compounds to $\mathrm{CO}_{2}$ and $\mathrm{H}_{2} \mathrm{O}$. However, in case of nitrogen-containing organic contaminants which are mainly produced in chemical and pharmacutical industries, the by products of the photocatalytic oxidation can be several inorganic forms of nitrogen such as ammonia $\left(\mathrm{NH}_{3}\right)$, nitrite $\left(\mathrm{NO}_{2}{ }^{-}\right)$and nitrate $\left(\mathrm{NO}_{3}{ }^{-}\right)$.

Reduction The presence of nitrate and/or nitrite ions in drinking water is a growing public health concern worldwide. According to U.S geological survey about $22 \%$ of domestic wells in united states has nitrate concentration over the maximum contaminant level (MCL) which $50 \mathrm{mg} / \mathrm{L}$ and $0.5 \mathrm{mg} / \mathrm{L}$ for nitrate and nitrite respectively [14]. The main causes of nitrate and/or nitrite groundwater pollution are use of synthetic fertilizers and disposal of municipal effluents by sludge which is spread on fields. Although nitrate $\left(\mathrm{NO}_{3}{ }^{-}\right)$does not show any toxicity its reduction to nitrite $\left(\mathrm{NO}_{2}{ }^{-}\right)$has an adverse biological effect. Nitrite $\left(\mathrm{NO}_{2}{ }^{-}\right)$ions oxidizes oxygen carriers hemoglobin to methemoglobin ren- 
dering it unable to transport oxygen to the tissues. When the concentration of methemoglobin exceeds $10 \%$ of the normal hemoglobin molecules the condition is called methemoglobinemia which is life threatening. This situation gets even more aggravated for infants (younger than 6 months) since they have hemoglobin molecules which are more susceptible to formation of the methemoglobin causing blue-baby syndrome.

\subsection{Heterogeneous Photocatalysis}

Heterogeneous photocatalytic reactions initiate by illumination of light on a solid semiconductor (SC). Semiconductors have two energy bands which are separated by the band gap energy. The highest energy band which is fully occupied by electrons is called the valence band (VB) and the lowest energy band which is empty from electrons is called conduction band (CB). When illuminated with light of energy equal or higher than the band gap energy $\left(E_{b g}\right)$ an electron $\left(e^{-}\right)$ excites from the VB to $\mathrm{CB}$ and leaving behind a hole $\left(\mathrm{h}^{+}\right)$(equation 1.1).

$$
\mathrm{SC}+\mathrm{h} \nu \longrightarrow \mathrm{e}_{\mathrm{cb}}^{-}+\mathrm{h}_{\mathrm{vb}}^{+}
$$

In case of titanium dioxide (anatase phase) as the photocatalyst semiconductor, the band gap is equal to $3.2 \mathrm{eV}$ corresponding to a wavelength of $\lambda=387 \mathrm{~nm}$.

The generated holes and electrons form the oxidizing agents required for the degradation of the organic contaminants through the following pathways [15].

\section{Hole-driven pathway:}

In this pathway (Eq.1.2) hydroxyl radicals are formed through the reaction between hydroxyl ions with the generated holes. Hydroxyl radicals are able to oxidatively destroy a wide range of organic contaminants in water (Eq.1.3) [5].

$$
\begin{aligned}
& \mathrm{h}_{\mathrm{vb}}{ }^{+}+\mathrm{OH}_{\mathrm{ads}}{ }^{-} \longrightarrow \mathrm{OH}_{\mathrm{ads}} \cdot \\
& \mathrm{OH}_{\mathrm{ads}} \cdot+\text { organic contaminants } \longrightarrow \text { oxidized species }
\end{aligned}
$$


Electron-driven pathway: In this pathway initially the generated electrons from the conductance band of the semiconductor react with molecular oxygen and form oxygen superoxide ions (Eq.1.4). Formation of hydrogen peroxide (Eq.1.6) contributes to the production of more hydroxyl radicals and more degradation of the organic contaminants as a consequence. The explained mechanism is schematically illustrated in figure 1.1 (a).

$$
\begin{aligned}
& \mathrm{e}_{\mathrm{cb}}^{-}+\mathrm{O}_{2, \text { ads }} \longrightarrow \mathrm{O}_{2}{ }^{--} \\
& \mathrm{O}_{2}^{\cdot-}+\mathrm{H}^{+} \longrightarrow \mathrm{HO}_{2} \cdot \\
& \mathrm{HO}_{2}{ }^{\cdot}+\mathrm{HO}_{2} \cdot \longrightarrow \mathrm{H}_{2} \mathrm{O}_{2}+\mathrm{O}_{2} \\
& \mathrm{H}_{2} \mathrm{O}_{2}+\mathrm{e}_{\mathrm{cb}}^{-} \longrightarrow \mathrm{OH}^{\cdot}+\mathrm{OH}^{-} \\
& \text {organic contaminants }+\mathrm{O}_{2}{ }^{-}\left(\text {or } \mathrm{OH}^{\cdot}\right) \longrightarrow \text { oxidized species }
\end{aligned}
$$

Wang et al. [16] evaluate the degradation of methylene blue in a microreactor where they selectively control the electron and hole driven pathways by biasing the conductive support in forward and reverse fashion. They reported that the electron-driven pathway is more efficient than the hole-driven pathway.

From the energy efficiency point of view, semiconductors with low bandgap energies are more desirable since lower light energy is required for excitation. However, low band gap materials are less stable and have a higher tendency for photoanodic corrosion [17]. Another important concern is the charge carriers recombination which has a detrimental effect on the photocatalytic performance. Several attempts have been made to reduce the recombination rate by improving charge carriers' separation. The most common method is introducing noble metals such as platinum $(\mathrm{Pt})$, gold $(\mathrm{Au})$ [18] palladium $(\mathrm{Pd})$ and silver $(\mathrm{Ag})$ [19] that act as electron scavenger minimizing recombination. Another novel approach is coupling carbonaceous materials such as carbon nanotubes and graphene [20] with the semiconductors. In this arrangement the excited electrons from semiconductors are transfered to graphene due to its conducting and semiconducting nature, thus lowering the recombination. 


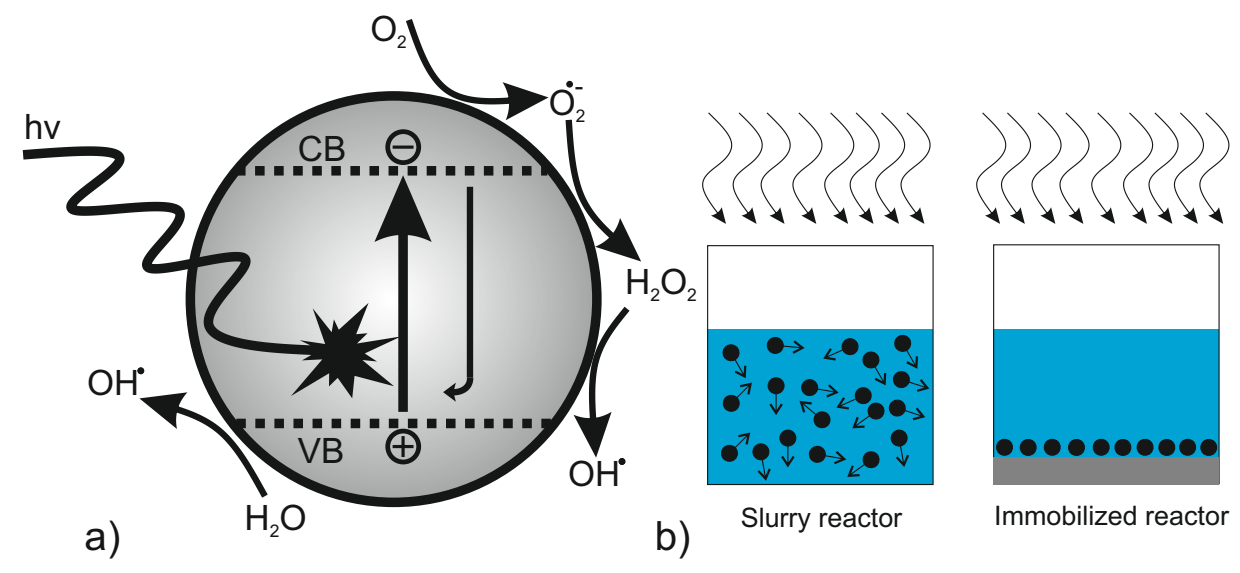

Figure 1.1: a) Upon the absorption of photons with equal or higher energy than the bandgap of a semiconductor, electrons are excited from the valence band (VB) to the conduction band (CB). Excited electrons are transfered to oxygen molecules forming superoxide ion radicals $\left({ }^{\cdot} \mathrm{O}_{2}{ }^{-}\right)$and electron transfer from water molecules to the VB hole forming hydroxyl radical $\left(\mathrm{OH}^{*}\right)$ At the same time some excited electrons recombine. b) Difference between a slurry and an immobilized reactor. In a slurry reactor, although the mass transfer is optimum,the photon transfer is sub-optimum due to the strong absorption and scattering of light by the suspended particles.

\subsubsection{Titanium Dioxide}

Many semiconductors such as $\mathrm{ZnO}, \mathrm{CdS}, \mathrm{Fe}_{2} \mathrm{O}_{3}$, etc can be utilized for photocatalysis but $\mathrm{TiO}_{2}$ is the most studied one. In addition to its favorable properties such as chemical stability (low photocorrsion) relatively cheap and being environmentally friendly, its electronic structure makes it an ideal semiconductor for photocatalytic applications. As figure 1.2 shows, the $\mathrm{TiO}_{2} \mathrm{CB}$ is higher than the oxidized state of water while its VB is lower in energy than water. In other words the redox potential of the valence band is positive enough to generate $\mathrm{OH}^{*}$ radicals. In addition $\mathrm{TiO}_{2}$ has some other favorable properties compared to other semiconductors. For instance, the charge carriers have a very short life time in $\mathrm{Fe}_{2} \mathrm{O}_{3}$ [21] while in $\mathrm{TiO}_{2}$ (anatase) they are reasonably long. $\mathrm{ZnO}$ is vulnerable to deactivation due to the formation of $\mathrm{Zn}(\mathrm{OH})_{2}$ on its surface [22] while $\mathrm{TiO}_{2}$ is stable during the photocatalytic reactions.

Titanium dioxide $\left(\mathrm{TiO}_{2}\right)$ has been commercially produced from the early twentieth century and has traditionally been utilized as additive to polymeric binders [23], 
toothpaste [24], and sunscreens [25]. After the discovery of water splitting on $\mathrm{TiO}_{2}$ electrodes upon UV illumination by Fujishima and Honda in 1972, $\mathrm{TiO}_{2}$ finds increasing applications in photovoltaics and photocatalysis. The earliest report on $\mathrm{TiO}_{2}$ based photocatalytic water purification was in 1977 by Frank and Bard [26]. They discussed the formation of cyanide to cyanate for detoxification of water.

Titanium dioxide has three well-known polymorphs at atmospheric pressure; rutile, anatase and brookite (figure 1.3). Rutile is the most stable polymorph and Brookite is less attractive due to the difficulty in synthesis and its limited instability [27]. The general properties of rutile and anatase phases are summarized in table 1.1. Although both phases have tetragonal crystal structures, rutile has a lower band gap compared to anatase. Several studies have documented that anatase has a higher photocatalytic activity compared to rutile. The reason behind this observation is subject of extensive research. For instance Banerjee et al. reported the indirect band gap and higher fermi level in anatase would contribute to a longer life time of photogenerated charge carries and higher photocatalytic activity [28].

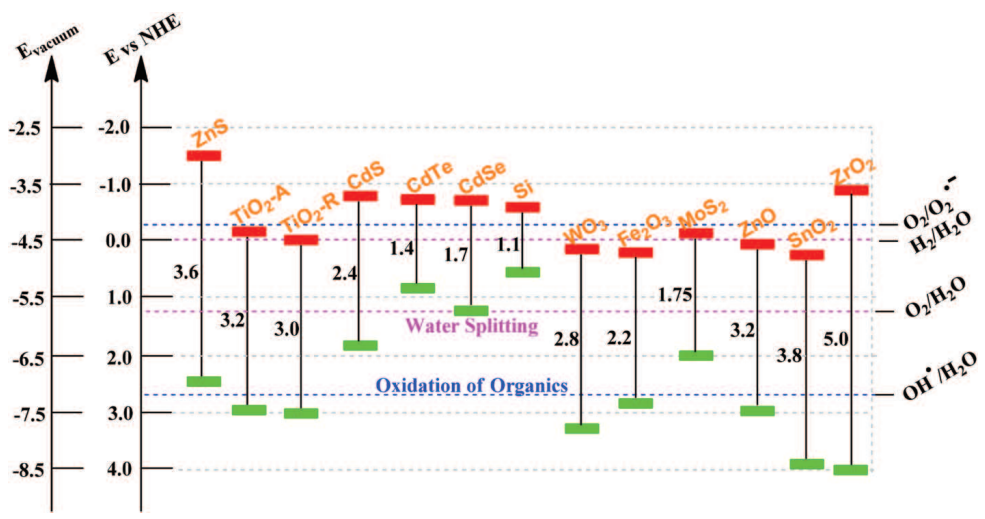

Figure 1.2: Band structure of different semiconductors vs energy for normal hydrogen electrode (NHE) or the vacuum level as reference. The lower edge of conduction band (red) and upper edge of valence band (green) along with the band gap energy are shown (adapted from [22]).

Titanium dioxide can be deposited by various methods. Typical examples include sol-gel [31], suspension coating, electrophoretic deposition (EPD) [32], electrochemical deposition [33], chemical vapor deposition (CVD) [34], electron 
a)

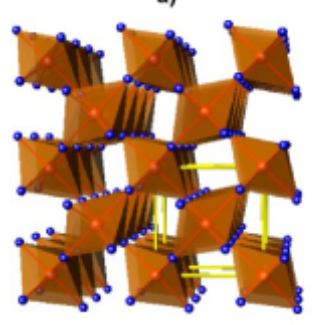

b)

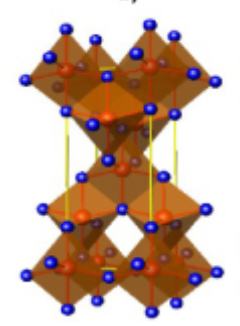

c)

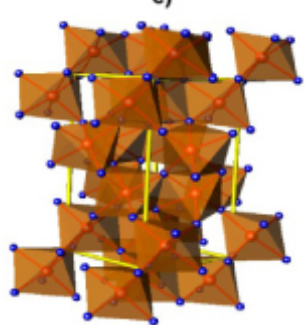

Figure 1.3: Crystal structure of $\mathrm{TiO}_{2}$ polymorphs. (a) rutile, (b) anatase and (c) brookite (adapted from [29]).

Table 1.1: General properties of rutile and anatase $\mathrm{TiO}_{2}$ polymorphs (adapted from $[30])$.

\begin{tabular}{lll}
\hline Property & Anatase & Rutile \\
\hline Crystal structure & Tetragonal & Tetragonal \\
& $\begin{array}{l}\mathrm{a}=0.3785 \\
\mathrm{c}=0.9514\end{array}$ & $\begin{array}{l}\mathrm{a}=0.4594 \\
\mathrm{c}=0.29589\end{array}$ \\
Lattice parameters & $\begin{array}{l}3.2(\mathrm{eV}) \\
(387 \mathrm{~nm})\end{array}$ & $\begin{array}{l}3.0(\mathrm{eV}) \\
(413 \mathrm{~nm})\end{array}$ \\
Band gap (wavelength) & 2.54 & 2.79 \\
\hline
\end{tabular}

beam evaporation [35], different sputtering configurations [36, 37], pulsed laser deposition (PLD) [38] and reactive magnetron sputtering (chapter 2 and 3). The methods like sol-gel and suspension coating are ideal for forming porous structures but limiting uniformity and mechanical stability are the drawbacks. Sputter deposition is ideal for catalysts requiring high uniformity and robustness. Furthermore, the morphology, composition and crystallinity can be accurately controlled by modifying the deposition conditions. Reactive DC magnetron sputtering has been employed in this thesis for deposition of $\mathrm{TiO}_{2}$ which will be briefly explained.

\section{D.C Magnetron sputtering}

D.C magnetron sputtering is a physical vapor deposition technique performed in vacuum condition $\left(\sim 10^{-3}\right.$ mbar $)$. In this process a DC electric field is ex- 
erted to a inert gas (Ar) at vacuum condition. As a result the inert gas becomes ionized and a glow discharge or plasma forms between the two electrodes. The cathode and anode are connected to the material $(\mathrm{Ti})$ which will be deposited (target) and substrate, respectively. The positively charged ions bombard the surface of the target material and eject atoms that form a thin film on the substrate. The anode could be biased negatively, heated or both according to the desired thin film properties. The presence of magnets behind the target causes the electrons to be trapped in a magnetic field close to the target. This firstly prevents the bombardment of the substrate by electrons which is the main drawback of diode sputtering (basic sputtering) and at the same time increases the chance of ionization more locally close to the target leading to higher sputtering rate. Various configuration of magnetron sputtering are available based on the position of the magnets such as cylindrical post magnetron, magnetron gun and planar magnetron [39]. Figure 1.4 illustrates the magnetron sputtering process schematically. Compound thin films can be sputtered either using DC magnetron sputtering with compound targets or DC "reactive" magnetron sputtering (chapter $2,3,4)$. The former is not generally desirable since fabrication of a metal target with acceptable purity is more convenient than a compound target with similar purity. In DC reactive magnetron sputtering, a second (reactive) gas is present which can react with the target. The addition of the reactive gas has a distinguished effect on the sputtering process and deposited thin films. At low flow rates, due to the consumption of the reactive gas by reaction with the internal chamber walls (gettering), the composition of the plasma and the deposited film is still metal-rich (metallic region). At a critical value of the reactive gas flow rate the composition of the target surface changes to one close to the compound (reactive region). Thin films formed at metallic and reactive regions are typically sub-stoichiometric and stoichiometric, respectively (chapter 2). In the case of $\mathrm{TiO}_{2}$ the target is titanium and the sputtering is performed in a mixture containing $\mathrm{O}_{2}$ as reactive gas and $\mathrm{Ar}$ as a working gas.

\subsubsection{Photocatalytic reactors}

Two significant parameters to be considered for the design of the photocatalytic reactor are photon and mass transfer. Photocatalytic reactors can be catego- 


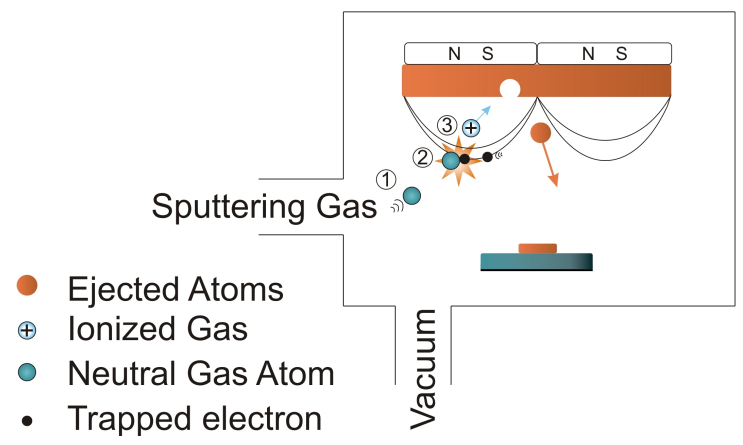

Figure 1.4: A schematic illustration of magnetron sputtering; 1) Argon gas is introduced into the chamber, 2) ever existing electrons trapped in the vicinity of the target ionize Argon gas forming plasma, 3) The plasma impinge the target and ejects atom from the surface. The ejected atoms accelerate towards substrate.

rized into two main groups according to the condition of the photocatalyst (figure 1.1(b)); The photocatalyst can be suspended in the reaction medium (slurry reactor) or immobilized to a support. The latter one is discussed in this thesis. In slurry reactors the catalyst particles are freely dispersed in the aqueous medium. This puts severe limitations on the light intensity distribution, but also requires a separation step afterwards. In immobilized systems the photocatalyst is attached to a fixed support. Shan et al. [40] provide a comprehensive study on the immobilization of $\mathrm{TiO}_{2}$ on supporting materials where they set requirements for the ideal support material as followings:

1. The attachment between catalyst and support should be robust

2. Should be chemically inert and remains intact after the photocatalytic processes

3. Providing high surface area which is beneficial for catalytic reactions

4. Having strong adsorption affinity towards the targeted contaminants

\section{Microreactors}

Immobilized systems have many advantages, but a low surface area to volume ratio compared to slurry systems is the main drawback. Microreactors have 
characteristic internal dimensions, e.g. fluid channels, in the micrometer to submillimeter range [41]. Due to the small dimension they have excellent heat and mass transfer which is highly beneficial for fast catalytic processes. In addition due to the small dimension the flow is laminar and easily described. The small volume also improves the process safety [41]. Table 1.2 compares different reactor configurations based on surface area to volume ratio. Microreactors posses a high surface area to volume ratio compared to other immobilized reactors. Besides microreactors are excellent platforms for catalyst screening due to the fast and efficient data analysis. With focus on photocatalysis they provide higher spatial illumination homogeneity. The throughput increase in microreactors is realized by numbering-up, meaning that the functional unit of a microreactor is simply repeated (figure 1.5 ).

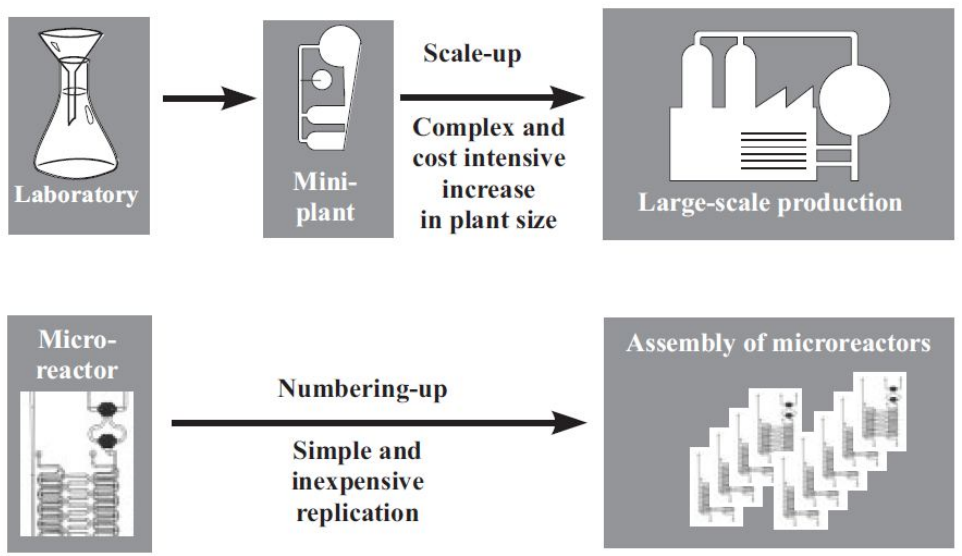

Figure 1.5: A comparison between conventional scale-up and numbering-up technique (adapted from [41]).

The transport and reaction in photocatalytic systems are summarized by the following steps:

1. Diffusion of the reactants from the bulk of fluid to the surface of the catalyst (including porous layers)

2. Adsorption of the reactants

3. Absorption of photons with required energy and formation of the electron holes pairs 
4. Transfer of electrons and holes to the adsorbed reactants

5. Reaction of the adsorbed species and formation of the products

6. Desorption of the products and diffusion into the bulk of fluid

Table 1.2: Comparison of different photoreactor configurations (adapted from [42]).

\begin{tabular}{|c|c|c|}
\hline $\begin{array}{l}\text { Photocatalytic } \\
\text { reactor }\end{array}$ & References & Surface area to volume ratio $\left(\mathrm{m}^{2} / \mathrm{m}^{3}\right)$ \\
\hline \multirow{5}{*}{ Slurry reactor } & {$[43]$} & 2631 \\
\hline & {$[44]$} & $8500-170000$ \\
\hline & {$[43]$} & 27 \\
\hline & {$[45]$} & 69 \\
\hline & {$[43]$} & 133 \\
\hline \multirow{6}{*}{$\begin{array}{l}\text { Annular/immersion } \\
\text { reactor }\end{array}$} & {$[46]$} & 170 \\
\hline & {$[47]$} & 340 \\
\hline & {$[43]$} & 2667 \\
\hline & {$[48]$} & 46 \\
\hline & {$[49]$} & 53 \\
\hline & {$[50]$} & 112 \\
\hline \multirow{4}{*}{ Optical fiber/hollow tube reactor } & {$[51]$} & 210 \\
\hline & $45]$ & 1087 \\
\hline & {$[52]$} & 1920 \\
\hline & {$[43]$} & 20000 \\
\hline \multirow{2}{*}{ Monolith reactor } & {$[53]$} & 1333 \\
\hline & {$[54]$} & $50-130$ \\
\hline \multirow{2}{*}{ Spinning disk reactor } & {$[55]$} & 7300 \\
\hline & {$[56]$} & 12000 \\
\hline \multirow{3}{*}{ Microreactor } & {$[57]$} & 23000 \\
\hline & {$[58]$} & 14000 \\
\hline & {$[59]$} & 250000 \\
\hline
\end{tabular}

\subsubsection{Kinetics of photocatalytic reactions}

The performance of a photocatalytic reactor is commonly assessed by considering a Langmuir-Hinshelwood (LH) kinetic model $r=-\frac{d c}{d t}=\frac{k K C}{1+K C}$ where $k, K$ and $C$ are the reaction rate constant, the equilibrium constant for adsorption of the target molecule and concentration of the target molecule, respectively. At low concentrations it is approximated by a first order reaction $r=-k c$ from which the overall reaction rate constant is extracted. As described earlier for 
heterogeneous catalysis, the first step concerns the diffusion of the reactants from the bulk to the surface of the catalyst. The photocatalytic reactions on the surface generate a concentration gradient between the bulk solution and the surface of the photocatalyst. The calculated reaction rate constant by this method is possibly affected by mass transfer for the studied reactant. For instance if the chemical reaction is faster than the diffusion of the reactant to the surface of the catalyst the overall reaction rate constant is lower than the intrinsic reaction rate constant. A conventional approach for extracting the intrinsic reaction rate constant is placing the system in the reaction limiting regime by using a differential reactor. A differential reactor consists of a reaction chamber and a mixing tank, where the reaction volume is much smaller than the total volume. The small conversion per pass allows the simplification of the mass balance to a batch reactor equation. The intrinsic kinetics are determined for flowrate - independent conversions [6071]. Accepting the reliability of this method to eliminate external mass transport, the question about internal mass transfer remains, which will be present even for thin catalyst layers or for an inevitable degree of aggregation in slurry systems. Ballari et al. $[72,73]$ went on to defining guidelines for canceling mass transfer limitations in slurry reactors based on flow rate, catalyst loading and irradiation rates.

Even when concentration gradients are eliminated, the inhomogeneity regarding light distribution has to be carefully considered, especially for slurry systems. Motegh et al. [74] gave guidelines for operating in an optically differential mode. Starting from the premise of perfect mixing, a criterion was defined for keeping the gradients in photon absorption rate small enough as to allow for volume-averaging of the reaction rate. A more reliable method is to model light distribution, fluid dynamics and mass transfer and fit the kinetics to the experimental data. Due to the complexity of large-scale photocatalytic reactors, a lot of assumptions come into play.

The first challenge in a large-scale system is the non-uniform incident flux. To obtain the radiation field distribution, light emission models have to be correlated to the radiation transfer equation (RTE) in order to obtain the local volumetric rate of energy absorption (LVREA) which can be afterwards coupled to the reaction rate. In case of dispersed systems, the radiation transfer equation 
becomes more complex due to in and out-scattering effects that also depend on the aggregation extent of the particles [75-79].

The next step is to consider the complex hydrodynamics. The most rigorous approach is to perform a CFD simulation which solves the continuity and NavierStokes equations. Again, dispersed systems demand the most elaborate models. An Eulerian multi-fluid approach is necessary to connect the fluid velocity field to the solid particle distribution. However, real flow computations are quite challenging. This is why, when possible, approximations are used. In case of small deviations from laminar flow, the axial dispersed model can be considered. For this, the Péclet number $(\mathrm{Pe})$ can be experimentally determined from residence time distribution (RTD) measurements [80, 81].

Once the velocity field is characterized, mass transport can be investigated. The most accepted approximation for slurry reactors is a one phase system with high Pe numbers. Hence, the governing equation becomes represented by advection and homogeneous reaction only $[82,83]$.

For immobilized systems it is easier to couple the reaction rate to mass transfer, given the clear definition for the interface. The most realistic transport models for the flow channel take into account both advection and diffusion [47, 84-87]. However in these studies the internal mass transport inside the catalyst film were not accounted for. Instead, the reaction rate was set as the boundary condition for the catalyst-fluid interface.

Microreactors are a special case. The modeling of such systems is straightforward due to their laminar flow and constant photon flux density throughout the entire surface of the reactor. Moreover, for immobilized catalyst, interface scattering due to roughness can often be neglected. Hence, the radiative transfer equation (RTE) simplifies to a Lambert-Beer law. This allows for a direct inclusion of the light distribution in a photocatalytic layer.

\subsection{Hydrogenation}

The conventional methods for nitrate or/and nitrite removal such as ion exchange, reverse osmosis and electrodialysis are not desirable due to high cost of operations and post disposal of the nitrate brine [88]. In these methods the ions are isolated 
instead of degraded to non-harmful products. The biological degradation through microorganisms is not efficient due to the long process time. The catalytic denitrification in aqueous environment through hydrogenation of the nitrite and/or nitrate over noble-metal solid catalysts is proven to be very efficient and cost effective. As shown below in this process, firstly nitrate is reduced to nitrite over a bimetallic catalyst. This is followed by nitrite reduction to ammonia and nitrogen in the subsequent reactions. Ammonia is an undesirable by-product thus high selectivity to nitrogen is desired. The selectivity towards nitrogen is affected by many factors including $\mathrm{pH}$, temperature and hydrogen/nitrogen ratio.

$$
\begin{aligned}
& 2 \mathrm{NO}_{3}{ }^{-}+2 \mathrm{H}_{2} \stackrel{P d-C u}{\longrightarrow} 2 \mathrm{NO}_{2}{ }^{-}+2 \mathrm{H}_{2} \mathrm{O} \\
& 2 \mathrm{NO}_{2}{ }^{-}+3 \mathrm{H}_{2} \stackrel{P d}{\longrightarrow} \mathrm{N}_{2}+4 \mathrm{H}_{2} \mathrm{O} \\
& \mathrm{NO}_{2}{ }^{-}+3 \mathrm{H}_{2}+2 \mathrm{H}^{+} \stackrel{P d}{\longrightarrow} \mathrm{NH}_{4}^{+}+2 \mathrm{H}_{2} \mathrm{O}
\end{aligned}
$$

\subsubsection{Membrane reactors}

Heterogeneous catalysis in multiphase reactors are conventionally performed in slurry phase, fixed/trickle bed and agitated tank reactors. In slurry reactors the separation of the catalyst particles and attrition are important drawbacks. In fixed bed reactors, although filtration is not required, the presence of large catalyst particles and as a result long diffusion paths remains an issue. Membrane reactors on the other hand have several advantages when it comes to heterogeneous catalytic processes. The interface between the gas and liquid phase flowing from opposite sides of membrane over the catalyst is well-defined while the membrane itself can act as a support for the catalyst [89]. Sylvian et al. [90] categorize catalytic membrane reactors according to the role of membrane as an extractor, distributor and contactor. In the extractors group, membranes function to remove the products which has detrimental effect on the kinetics from the reaction zone. In the distributors category the role of the membrane is distributing the reactants homogeneously over the catalytic region. Finally in the contactors, the membrane provides an optimum contact between the reactants and catalyst. The 


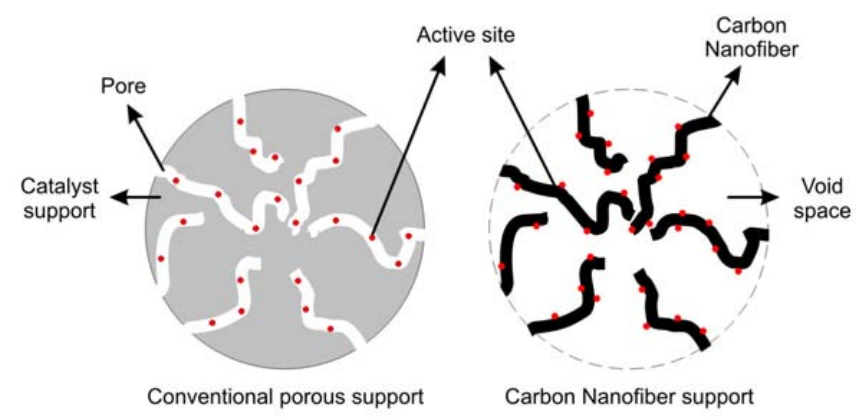

Figure 1.6: CNFs based catalyst support compared with conventional porous support (adapted from [94]).

reactants could either separately be fed from both sides of the membrane or flow mixed inside the reactor. Inorganic materials are favorable in catalytic reactions due to their stability at high temperature and harsh environment for catalyst immobilization and regeneration [91].

\subsubsection{Carbon nanofiber based catalyst support}

Hydrogenation of nitrite by heterogeneous catalysis is significantly fast causing frequently mass transfer limitation. Although microreactors due to short characteristic lengths are beneficial for these processes, the surface area to volume ratio is limited for these systems. CNF catalyst supports are very efficient due to their high pore volume, high surface and low tortuosity lowering internal mass transfer limitation. Figure 1.6 clearly shows that CNF based supports have an inverse structure of a conventional porous support material providing an open structure which is advantageous in terms of porosity and tortuosity. In addition CNFs posses excellent mechanical stability (high young's modulus) [92] and chemically inertness [93].

Carbon nanofibers (CNF) are one of the main allotropes of carbon. CNFs are made of curved graphite layers stacked on top of each other forming two different morphologies; herringbone and bamboo type fibers (figure 1.7). CNFs are synthesized by different methods including arc discharge, laser ablation, catalytic chemical vapour deposition (c-CVD) and catalytic plasma enhanced chemical vapour deposition (c-PECVD) [95]. For these reasons, CNFs as the catalyst 
supports have been utilized in many studies. Puron et al. [96] employed CNFs supported catalyst for heavy oil hydroprocessing where they observed higher conversions compared to bare $\mathrm{Al}_{2} \mathrm{O}_{3}$ catalyst supports. Ermenko et al. [97] reported the advantageous role of CNFs supported catalyst $(\mathrm{Pd})$ for hydrogenation of nitrocompounds to amines. They observed high activity due to the high palladium particles dispersity when CNFs used as the catalyst support.
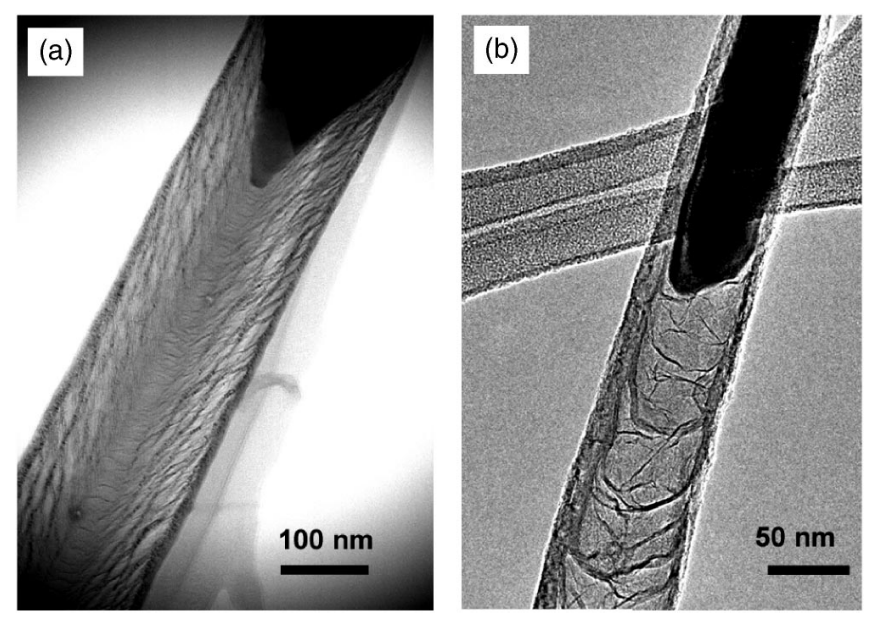

Figure 1.7: (a) STEM image of a herringbone carbon nanofiber and (b) TEM image of a bamboo type carbon nonofiber (adapted from [95]). 


\subsection{Thesis outline}

Chapter 2, discusses the formation of $\mathrm{TiO}_{2}$ thin films via $\mathrm{DC}$ reactive magnetron sputtering. The effect of oxygen concentration during sputtering deposition on the final film structure and properties is investigated. The dynamics of the annealing process were followed by in situ ellipsometry, showing the optical properties transformation. The final crystal structures are identified by XRD. Finally the charge carrier mobility was measured by time-resolved microwave conductance.

Chapter 3, demonstrates the synthesis and optical characterization of $\mathrm{TiO}_{2}$ thin films. The optimized $\mathrm{TiO}_{2}$ thin films was incorporated in a microreactor and the photocatalytic performance was assessed using methylene blue as model compound. The intrinsic photocatalytic activity of the catalysts was evaluated using a numerical model by which the intrinsic reaction rate constants were extracted. Moreover, the photocatalytic activity as a function of thin film thickness was investigated.

Chapter 4, presents the effect of different substrates on the surface charge of $\mathrm{TiO}_{2}$ and the photocatalytic performance. Patterned $\mathrm{TiO}_{2}$ thin films were fabricated and an Atomic Force Microscopy (AFM) is employed to measure in-situ force interaction between the AFM tip and $\mathrm{TiO}_{2}$ deposited on different substrates with and without illumination. The local surface charge was extracted from the force measurements.

Chapter 5, explains the fabrication and modeling of an immobilized porous $\mathrm{TiO}_{2}$ thin film based photocatalytic microreactor. A numerical model is built for both light independent and light dependent of first order kinetics. Experimental data for various residence times, catalyst thicknesses and photon flux densities were investigated and compared with the results of the model. Furthermore, a criterion is defined based on the absorption coefficient and catalyst thickness to mark the transition towards the regime where the incorporation of photon flux density is required. Performance parameters are also derived for the light depen- 
dent model for which the internal effectiveness factor reveals both mass transfer and light limitations.

Chapter 6, describes fabrication and catalytic performance of an alumina hollow fiber microreactor impregnated with carbon nanofibers as catalyst support for hydrogenation of nitrites. In addition the performance of this microreactor was compared with different reactor configurations. The reaction selectivity to ammonia, the undesirable product was discussed and optimized.

Chapter 7, concludes the thesis. A summary of the thesis is presented and an outlook on future experimental studies is given. 



\section{Bibliography}

[1] U. Nations and C. Singer, Journal of government information (United Nations Development Programme, 2006), No. 6, pp. 839-840.

[2] B. Ingram, Urban urgency, Supermarket Business 54, 1999 (1999).

[3] W. H. O. Library, C. Data, M. D. Goals, U. Joint, M. Programme, W. Supply, W. H. O. Press, A. Appia, and N. York, World Health Organization and UNICEF 2010, World Health 1, 60 (2010).

[4] UN-Water, Coping with Water Scarcity: Challenge of the Twenty-First Century, 1 (2007).

[5] R. Andreozzi, Advanced oxidation processes (AOP) for water purification and recovery, Catalysis Today 53, 51 (1999).

[6] J. Kuipers, H. Bruning, D. Yntema, and H. Rijnaarts, Wirelessly powered ultraviolet light emitting diodes for photocatalytic oxidation, Journal of Photochemistry and Photobiology A: Chemistry 299, 25 (2015).

[7] R. Munter, Advanced Oxidation Processes - Current Status and Prospect, Proc. Estonian Acad. Sci. Chem. 50, 59 (2001).

[8] U. von Gunten and J. Hoigné, Bromate formation during ozonation of bromide-containing waters: interaction of ozone and hydroxyl radical reactions, Environ. Sci .TechnolTechnol 28, 1234 (1994).

[9] M. B. Ray, J. P. Chen, L. K. Wang, and S. O. Pehkonen, in Treatment Technologies for Removal of Methyl Tertiary Butyl Ether (MTBE) from Drinking Water, edited by 2nd (Center for Groundwater Restoration and Protection National Water Research Institute, 2000).

[10] F. Haber and J. Weiss, The Catalytic Decomposition of Hydrogen Peroxide by Iron Salts, Proceedings of the Royal Society of London. Series A, Mathematical and Physical Sciences 147, 332 (1934).

[11] M. J. Benotti, R. a. Trenholm, B. J. Vanderford, J. C. Holady, B. D. Stan- 
ford, and S. a. Snyder, Pharmaceuticals and endocrine disrupting compounds in U.S. drinking water., Environmental science \& technology 43, 597 (2009).

[12] U. Diebold, The surface science of titanium dioxide, Surface Science Reports 48, 53 (2003).

[13] M. Borges, D. García, T. Hernández, J. Ruiz-Morales, and P. Esparza, Supported Photocatalyst for Removal of Emerging Contaminants from Wastewater in a Continuous Packed-Bed Photoreactor Configuration, Catalysts 5, 77 (2015).

[14] M. H. Ward, T. M. DeKok, P. Levallois, J. Brender, G. Gulis, B. T. Nolan, and J. VanDerslice, Workgroup report: Drinking-water nitrate and health Recent findings and research needs, Environmental Health Perspectives 113, 1607 (2005).

[15] J.-M. Herrmann, Photocatalysis fundamentals revisited to avoid several misconceptions, Applied Catalysis B: Environmental 99, 461 (2010).

[16] X. Zhang, Photocatalytic microreactors for water purification: Selective control of oxidation pathways, The 8th Annual IEEE International Conference on Nano/Micro Engineered and Molecular Systems 1, 368 (2013).

[17] J. Kumar and A. Bansal, Photocatalysis by Nanoparticles of Titanium Dioxide for Drinking Water Purification: A Conceptual and State-of-Art Review, Materials Science Forum 764, 130 (2013).

[18] S. H. Kim, C. H. Jung, N. Sahu, D. Park, J. Y. Yun, H. Ha, and J. Y. Park, Catalytic activity of $\mathrm{Au} / \mathrm{TiO}_{2}$ and $\mathrm{Pt} / \mathrm{TiO}_{2}$ nanocatalysts prepared with arc plasma deposition under $\mathrm{CO}$ oxidation, Applied Catalysis A: General 454, 53 (2013).

[19] W. Ziemkowska, D. Basiak, P. Kurtycz, A. Jastrzbska, A. Olszyna, and A. Kunicki, Nano-titanium oxide doped with gold, silver, and palladium synthesis and structural characterization, Chemical Papers 68, 959 (2014).

[20] D. Zhao, G. Sheng, C. Chen, and X. Wang, Enhanced photocatalytic degradation of methylene blue under visible irradiation on graphene@ $\mathrm{TiO}_{2}$ dyade structure, Applied Catalysis B: Environmental 111-112, 303 (2012).

[21] I. Paramasivam, H. Jha, N. Liu, and P. Schmuki, A Review of Photocatalysis using Self-organized $\mathrm{TiO}_{2}$ Nanotubes and Other Ordered Oxide Nanostructures, Small 8, 3073 (2012). 
[22] W. Wu, C. Jiang, and V. A. L. Roy, Recent Progress in Magnetic Iron Oxide Semiconductor Composite Nanomaterials as Promising Photocatalysts, Nanoscale 7, 38 (2014).

[23] G. Pfaff and P. Reynders, Angle-Dependent Optical Effects Deriving from Submicron Structures of Films and Pigments, Chemical Reviews (Washington, DC, United States) 99, 1963 (1999).

[24] Q. Li, S. Mahendra, D. Y. Lyon, L. Brunet, M. V. Liga, D. Li, and P. J. J. Alvarez, Antimicrobial nanomaterials for water disinfection and microbial control: Potential applications and implications, Water Research 42, 4591 (2008).

[25] R. Dunford, A. Salinaro, L. Cai, N. Serpone, S. Horikoshi, H. Hidaka, and J. Knowland, Chemical oxidation and DNA damage catalysed by inorganic sunscreen ingredients, FEBS Letters 418, 87 (1997).

[26] A. J. B. Steven N. Frank, Heterogeneous photocatalytic oxidation of cyanide ion in aqueous solutions at titanium dioxide powder, Journal of the american chemical society 99, 303 (1977).

[27] S. B. I. N. Park, The crystalline phase stability of titania particles prepared at room temperature by the sol-gel method, Journal of Materials Science 6, 4299 (2001).

[28] S. Banerjee and J. Gopal, Physics and chemistry of photocatalytic titanium dioxide: visualization of bactericidal activity using atomic force microscopy, Current Science 90, 1378 (2006).

[29] V. C. Fuertes, C. F. a. Negre, M. B. Oviedo, F. P. Bonafé, F. Y. Oliva, and C. G. Sánchez, A theoretical study of the optical properties of nanostructured $\mathrm{TiO}_{2}$., Journal of physics. Condensed matter : an Institute of Physics journal 25, 115304 (2013).

[30] D. a. H. Hanaor and C. C. Sorrell, Review of the anatase to rutile phase transformation, Journal of Materials Science 46, 855 (2010).

[31] P. Kajitvichyanukul, J. Ananpattarachai, and S. Pongpom, Sol-gel preparation and properties study of $\mathrm{TiO}_{2}$ thin film for photocatalytic reduction of chromium(VI) in photocatalysis process, Science and Technology of Advanced Materials 6, 352 (2005).

[32] W. Jarernboon, S. Pimanpang, S. Maensiri, E. Swatsitang, and V. 
Amornkitbamrung, Optimization of titanium dioxide film prepared by electrophoretic deposition for dye-sensitized solar cell application, Thin Solid Films 517, 4663 (2009).

[33] A. Manivannan, N. Spataru, K. Arihara, and A. Fujishima, Electrochemical Deposition of Titanium Oxide on Boron-Doped Diamond Electrodes, Electrochemical and Solid-State Letters 8, C138 (2005).

[34] T. Maruyama and S. Arai, Titanium dioxide thin films prepared by chemical vapor deposition, Solar Energy Materials and Solar Cells 26, 323 (1992).

[35] T.-S. Yang, C.-B. Shiu, and M.-S. Wong, Structure and Hydrophilicity of Titanium Oxide Films prepared by Electron Beam Evaporation, Surface Science 548, 75 (2004).

[36] L. M. Williams, Structural properties of titanium dioxide films deposited in an rf glow discharge, Journal of Vacuum Science \& Technology, A: Vacuum, Surfaces, and Films 1, 1810 (1983).

[37] T. Kubart, J. Jensen, T. Nyberg, L. Liljeholm, D. Depla, and S. Berg, Influence of the target composition on reactively sputtered titanium oxide films, Vacuum 83, 1295 (2009).

[38] Y. Suda, H. Kawasaki, T. Ueda, and T. Ohshima, Preparation of high quality nitrogen doped $\mathrm{TiO}_{2}$ thin film as a photocatalyst using a pulsed laser deposition method, Thin Solid Films 453-454, 162 (2004).

[39] M. Ohring, Materials science (Elsevier, 2001), pp. 95-201.

[40] A. Y. Shan, T. I. M. Ghazi, and S. A. Rashid, Immobilisation of titanium dioxide onto supporting materials in heterogeneous photocatalysis: A review, Applied Catalysis A: General 389, 1 (2010).

[41] W. Ehrfeld, V. Hessel, and H. Löwe, Microreactors - new technology for modern chemistry (Wiley, 2001), No. 1, pp. 89-89.

[42] T. Van Gerven, G. Mul, J. Moulijn, and A. Stankiewicz, A review of intensification of photocatalytic processes, Chemical Engineering and Processing: Process Intensification 46, 781 (2007).

[43] P. S. Mukherjee and A. K. Ray, Major Challenges in the Design of a LargeScale Photocatalytic Reactor for Water Treatment, Chemical Engineering \& Technology 22, 253 (1999).

[44] V. Brezová, E. Brandšteterová, M. Čeppan, and J. Pieš, Photocatalytic Ox- 
idation of p-Cresol in Aqueous Titanium Dioxide Suspension, Collection of Czechoslovak Chemical Communications 58, 1285 (1993).

[45] A. K. Ray and A. a.C.M Beenackers, Development of a new photocatalytic reactor for water purification, Catalysis Today 40, 73 (1998).

[46] T. H. Lim and S. D. Kim, Trichloroethylene degradation by photocatalysis in annular flow and annulus fluidized bed photoreactors, Chemosphere 54, 305 (2004).

[47] G. E. Imoberdorf, H. A. Irazoqui, O. M. Alfano, and A. E. Cassano, Scalingup from first principles of a photocatalytic reactor for air pollution remediation, Chemical Engineering Science 62, 793 (2007).

[48] W. Wang and Y. Ku, Photocatalytic degradation of gaseous benzene in air streams by using an optical fiber photoreactor, Journal of Photochemistry and Photobiology A: Chemistry 159, 47 (2003).

[49] J. C. S. Wu, H. M. Lin, and C. L. Lai, Photo reduction of $\mathrm{CO}_{2}$ to methanol using optical-fiber photoreactor, Applied Catalysis A: General 296, 194 (2005).

[50] A. Danion, J. Disdier, C. Guillard, F. Abdelmalek, and N. Jaffrezic-Renault, Characterization and study of a single-TiO ${ }_{2}$-coated optical fiber reactor, Applied Catalysis B: Environmental 52, 213 (2004).

[51] M. R. Hoffmann and M. M. K. Laboratories, Development and Optimiza-

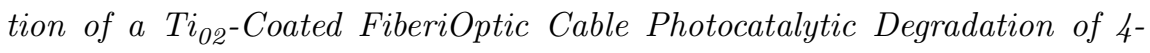
Chlorophenol, Enviromental science and technology 29, 2974 (1995).

[52] K. Hofstadler, R. Bauer, S. Novalic, and G. Heisler, New Reactor Design for Photocatalytic Wastewater Treatment with $\mathrm{TiO}_{2}$ Immobilized on FusedSilica Glass Fibers: Photomineralization of 4-Chlorophenol, Environmental Science \& Technology 28, 670 (1994).

[53] H. Lin and K. T. Valsaraj, An optical fiber monolith reactor for photocatalytic wastewater treatment, AIChE Journal 52, 2271 (2006).

[54] D. D. Dionysiou, G. Balasubramanian, M. T. Suidan, A. P. Khodadoust, I. Baudin, and J.-M. Lan, Rotating disk photocatalytic reactor: development, characterization, and evaluation for the destruction of organic pollutants in water, Water Research 34, 2927 (2000).

[55] Barthe and P. J., Method and microfludic reator for photocatalysis, 2004. 
[56] R. Gorges, Photocatalysis in microreactors, Journal of Photochemistry and Photobiology A: Chemistry 167, 95 (2004).

[57] D. Rafieian, R. T. Driessen, W. Ogieglo, and R. G. Lammertink, Intrinsic Photocatalytic Assessment of Reactively Sputtered $\mathrm{TiO}_{2}$ Films, ACS Applied Materials \& Interfaces 7, 8727 (2015).

[58] Y. Matsushita, . A. S. Kumada, K. Wakabayashi, K. Sakeda, and T. Ichimura, Photocatalytic Reduction in Microreactors, Chemistry Letters 35, 410 (2006).

[59] G. Takei, T. Kitamori, and H. B. Kim, Photocatalytic redox-combined synthesis of l-pipecolinic acid with a titania-modified microchannel chip, Catalysis Communications 6, 357 (2005).

[60] M. L. Satuf, M. J. Pierrestegui, L. Rossini, R. J. Brandi, and O. M. Alfano, Kinetic modeling of azo dyes photocatalytic degradation in aqueous $\mathrm{TiO}_{2}$ suspensions. Toxicity and biodegradability evaluation, Catalysis Today 161, 121 (2011).

[61] M. L. Satuf, R. J. Brandi, A. E. Cassano, and O. M. Alfano, Scaling-up of slurry reactors for the photocatalytic degradation of 4-chlorophenol, Catalysis Today 129, 110 (2007).

[62] C. Passalía, O. M. Alfano, and R. J. Brandi, A methodology for modeling photocatalytic reactors for indoor pollution control using previously estimated kinetic parameters, Journal of Hazardous Materials 211-212, 357 (2012).

[63] J. Marugan, R. van Grieken, C. Pablos, M. L. Satuf, A. E. Cassano, and O. M. Alfano, Modeling of a bench-scale photocatalytic reactor for water disinfection from laboratory-scale kinetic data, Chemical Engineering Journal 224, 39 (2013).

[64] J. Marugán, R. van Grieken, A. E. Cassano, and O. M. Alfano, Scaling-up of slurry reactors for the photocatalytic oxidation of cyanide with $\mathrm{TiO}_{2}$ and silica-supported $\mathrm{TiO}_{2}$ suspensions, Catalysis Today 144, 87 (2009).

[65] J. Marugán, R. van Grieken, A. E. Cassano, and O. M. Alfano, Intrinsic kinetic modeling with explicit radiation absorption effects of the photocatalytic oxidation of cyanide with $\mathrm{TiO}_{2}$ and silica-supported $\mathrm{TiO}_{2}$ suspensions, Applied Catalysis B: Environmental 85, 48 (2008).

[66] N. Doucet, O. Zahraa, and M. Bouchy, Kinetics of the photocatalytic degra- 
dation of benzene, Catalysis Today 122, 168 (2007).

[67] J. E. Duran, M. Mohseni, and F. Taghipour, Modeling of annular reactors with surface reaction using computational fluid dynamics (cfd), Chemical Engineering Science 65, 1201 (2010).

[68] M. Mohseni and F. Taghipour, Experimental and CFD analysis of photocatalytic gas phase vinyl chloride (VC) oxidation, Chemical Engineering Science 59, 1601 (2004).

[69] J. Moreira, B. Serrano, A. Ortiz, and H. de Lasa, A unified kinetic model for phenol photocatalytic degradation over TiO 2 photocatalysts, Chemical Engineering Science 78, 186 (2012).

[70] M. A. Mueses, F. Machuca-Martinez, and G. Li Puma, Effective quantum yield and reaction rate model for evaluation of photocatalytic degradation of water contaminants in heterogeneous pilot-scale solar photoreactors, Chemical Engineering Journal 215-216, 937 (2013).

[71] R. L. Pozzo, R. J. Brandi, A. E. Cassano, and M. a. Baltanás, Photocatalytic oxidation of oxalic acid in dilute aqueous solution, in a fully illuminated fluidized bed reactor, Chemical Engineering Science 65, 1345 (2010).

[72] M. D. L. M. Ballari, O. M. Alfano, and A. E. Cassano, Mass transfer limitations in slurry photocatalytic reactors: Experimental validation, Chemical Engineering Science 65, 4931 (2010).

[73] M. D. L. M. Ballari, R. Brandi, O. Alfano, and A. Cassano, Mass transfer limitations in photocatalytic reactors employing titanium dioxide suspensions. II. External and internal particle constrains for the reaction, Chemical Engineering Journal 136, 242 (2008).

[74] M. Motegh, J. Cen, P. W. Appel, J. R. van Ommen, and M. T. Kreutzer, Photocatalytic-reactor efficiencies and simplified expressions to assess their relevance in kinetic experiments, Chemical Engineering Journal 208, 607 (2012).

[75] C. a. Arancibia-Bulnes and S. a. Cuevas, Modeling of the radiation field in a parabolic trough solar photocatalytic reactor, Solar Energy 76, 615 (2004).

[76] R. J. Brandi, M. a. Citroni, O. M. Alfano, and A. E. Cassano, Absolute quantum yields in photocatalytic slurry reactors, Chemical Engineering Science 58, 979 (2003). 
[77] G. E. Imoberdorf, F. Taghipour, M. Keshmiri, and M. Mohseni, Predictive radiation field modeling for fluidized bed photocatalytic reactors, Chemical Engineering Science 63, 4228 (2008).

[78] G. Li Puma and A. Brucato, Dimensionless analysis of slurry photocatalytic reactors using two-flux and six-flux radiation absorption-scattering models, Catalysis Today 122, 78 (2007).

[79] G. Li Puma, Modeling of Thin-Film Slurry Photocatalytic Reactors Affected by Radiation Scattering, Environmental Science and Technology 37, 5783 (2003).

[80] F. Jović, V. Kosar, V. Tomašić, and Z. Gomzi, Non-ideal flow in an annular photocatalytic reactor, Chemical Engineering Research and Design 90, 1297 (2012).

[81] K. Demeestere, A. D. Visscher, J. Dewulf, M. V. Leeuwen, and H. V. Langenhove, A new kinetic model for titanium dioxide mediated heterogeneous photocatalytic degradation of trichloroethylene in gas-phase, Applied Catalysis B: Environmental 54, 261 (2004).

[82] G. Li Puma, P. L. Yue, and G. L. Puma, A Novel Fountain Photocatalytic Reactor for Water Treatment and Purification: Modeling and Design, Industrial \& Engineering Chemistry Research 40, 5162 (2001).

[83] G. L. Puma and P. L. Yue, A laminar falling film slurry photocatalytic reactor. Part II - Experimental validation of the model, Chemical Engineering Science 53, 3007 (1998).

[84] G. E. Imoberdorf, A. E. Cassano, H. A. Irazoqui, and O. M. Alfano, Optimal design and modeling of annular photocatalytic wall reactors, Catalysis Today 129, 118 (2007).

[85] V. Tomašić, F. Jović, and Z. Gomzi, Photocatalytic oxidation of toluene in the gas phase: Modelling an annular photocatalytic reactor, Catalysis Today 137, 350 (2008).

[86] I. Salvadó-Estivill, A. Brucato, and G. L. Puma, Two-dimensional modeling of a flat-plate photocatalytic reactor for oxidation of indoor air pollutants, Industrial and Engineering Chemistry Research 46, 7489 (2007).

[87] Z. Wang, J. Liu, Y. Dai, W. Dong, S. Zhang, and J. Chen, Cfd modeling of a uv-led photocatalytic odor abatement process in a continuous reactor, 
Journal of Hazardous Materials 216, 25 (2012).

[88] M. Shrimali and K. Singh, New methods of nitrate removal from water, Environmental Pollution 112, 351 (2001).

[89] M. Vospernik, a. Pintar, G. Berčič, J. Batista, and J. Levec, Potentials of Ceramic Membranes as Catalytic Three-Phase Reactors, Chemical Engineering Research and Design 82, 659 (2004).

[90] S. Miachon and J.-a. Dalmon, Catalysis in membrane reactors : what about the catalyst?, Topics in Catalysis 29, 59 (2004).

[91] G. Centi, R. Dittmeyer, S. Perathoner, and M. Reif, Tubular Inorganic catalytic membrane reactors: advantages and performance in multiphase hydrogenation reactions, Catalysis Today 79-80, 139 (2003).

[92] F. a. Ghavanini, H. Jackman, P. Lundgren, K. Svensson, and P. Enoksson, Direct measurement of bending stiffness and estimation of Young's modulus of vertically aligned carbon nanofibers, Journal of Applied Physics 113, 194308 (2013).

[93] K. P. De Jong and J. W. Geus, Carbon Nanofibers: Catalytic Synthesis and Applications, Catalysis Reviews 42, 481 (2000).

[94] J. K. Chinthaginjala, K. Seshan, and L. Lefferts, Preparation and Application of Carbon-Nanofiber Based Microstructured Materials as Catalyst Supports, Industrial \& Engineering Chemistry Research 46, 3968 (2007).

[95] a. V. Melechko, V. I. Merkulov, T. E. McKnight, M. A. Guillorn, K. L. Klein, D. H. Lowndes, and M. L. Simpson, Vertically aligned carbon nanofibers and related structures: Controlled synthesis and directed assembly, Journal of Applied Physics 97, 041301 (2005).

[96] H. Purón, J. Pinilla, I. Suelves, and M. Millan, Acid treated carbon nanofibers as catalytic support for heavy oil hydroprocessing, Catalysis Today 249, 79 (2015).

[97] N. K. Eremenko, O. Y. Podyacheva, Z. R. Ismagilov, and I. I. Obraztsova, Highly Dispersed Palladium on Carbon Nanofibers for Hydrogenation of $\mathrm{Ni}$ trocompounds to Amines, Eurasian Chemico-Technological Journal 17, 101 (2015). 



\section{CHAPTER 2}

\section{Selective deposition of anatase and rutile $\mathrm{TiO}_{2}$}

In this chapter we discuss the formation of $\mathrm{TiO}_{2}$ thin films via DC reactive magnetron sputtering. The oxygen concentration during sputtering deposition proved to be a crucial parameter with respect to the final film structure and properties. The initial deposition provided amorphous films that crystallise upon annealing to anatase or rutile, depending on the initial sputtering conditions. Substoichiometric films $\left(\mathrm{TiO}_{\mathrm{x}<2}\right)$, obtained by sputtering at relatively low oxygen concentration, formed rutile upon annealing in air, whereas stoichiometric films formed anatase. This route therefore presents a formation route for rutile films via lower $\left(<500{ }^{\circ} \mathrm{C}\right)$ temperature pathways. The dynamics of the annealing process were followed by in situ ellipsometry, showing the optical properties transformation. The final crystal structures were identified by XRD. The anatase film obtained by this deposition method displayed high carriers mobility as measured by time-resolved microwave conductance. This also confirms the high photocatalytic activity of the anatase films.

This chapter has been published as: Damon Rafieian, Wojciech Ogieglo, Tom Savenije and Rob G. H. Lammertink, Controlled formation of Anatase and Rutile $\mathrm{TiO}_{2}$ Thin films by Reactive Magnetron Sputtering, AIP Advances, 5, 097168 (2015), DOI:http://dx.doi.org/10.1063/1.4931925. 


\subsection{Introduction}

Titanium dioxide $\left(\mathrm{TiO}_{2}\right)$ has been commercially produced from the early twentieth century and has traditionally been utilised as additives to polymeric binders [1], toothpaste [2], and sunscreens [3]. In recent years, there has been an increasing interest in applications of $\mathrm{TiO}_{2}$ related to environmental remediation [4], energy generation [5] and biomedicine [6].

$\mathrm{TiO}_{2}$ has three well-known polymorphs at atmospheric pressure: rutile, anatase and brookite. Brookite is hardly studied due to its metastable crystal structure and according difficulty in synthesis. The research to date has focused on anatase and rutile polymorphs instead. The properties of $\mathrm{TiO}_{2}$ significantly depend on the microstructure and crystallographic phase. For instance anatase finds application in photovoltaics [7], electrodes for Li-ion batteries [8] and photocatalysis [9] for water and air purification. Rutile, due to its higher refractive index, is mostly studied for optoelectronics, semicondoctor electronics [10] and optical coatings [11]. Hence controlling the crystalline structure of $\mathrm{TiO}_{2}$ is of paramount importance.

Titanium dioxide thin films can be synthesized by techniques including solgel [12], suspension coating [13], electron beam evaporation [14], electrochemical deposition [15], sputtering [16, 17], pulsed laser deposition (PLD) [18] and many other methods $[19,20]$. Among these, reactive sputtering provides accurate control regarding composition and morphology. The resulting $\mathrm{TiO}_{2}$ thin films present high uniformity over large areas which makes them attractive for both industrial applications and fundamental studies.

Regardless the synthesis route, the initial crystalline $\mathrm{TiO}_{2}$ phase is usually the metastable anatase due to the faster recrystallization caused by its lower surface free energy compared to rutile. Generally, it is only possible to synthesize rutile at low temperatures by hydrothermal methods and precipitation of crystalline $\mathrm{TiO}_{2}$ [21-23]. Besides, rutile can be obtained through high-temperature treatment, above $600{ }^{\circ} \mathrm{C}$, by the non-reversible transformation of anatase to rutile (ART) [24-28].

Besides ART, the rutile phase of $\mathrm{TiO}_{2}$ was obtained by applying a negative bias on the substrate during reactive sputtering [29] or by modifying the RF 
power [30]. In addition, there are some attempts to modify the crystalline phase after deposition, e.g. by $\mathrm{N}^{+}$ion implantation [31].

Here, we study the formation of rutile and anatase polymorphs of titanium dioxide by controlling the oxygen flow rate during DC reactive magnetron sputtering followed by annealing in air. The annealing process is analyzed through insitu monitoring the ellipsometric parameter $(\psi)$ for both sub-stoichiometric and stoichiometric $\mathrm{TiO}_{2}$ using spectroscopic ellipsometry (SE). The optoelectronic properties of the films were studied using time resolved microwave conductance (TRMC) and were related to the photocatalytic characteristics [9].

\subsection{Experimental}

\subsubsection{Deposition of $\mathrm{TiO}_{2}$ thin film}

Magnetron reactive sputtering deposition was conducted at constant target DC power of $500 \mathrm{~W}$ and constant process pressure of $6 \times 10^{-3}$ mbar. The target was pre-sputtered for 2 minutes with a closed shutter. The substrate-target distance was set at $4.4 \mathrm{~cm}$ and the substrate was rotated at $5 \mathrm{rpm}$ during the whole deposition process for enhanced uniformity. Thin films were sputtered on silicon p-type (100) substrates in $\mathrm{Ar} / \mathrm{O}_{2}$ atmosphere with additional controlled oxygen flow rate. The thickness of the deposited thin films were $\sim 200 \mathrm{~nm}$. All of the depositions were performed at room temperature without any substrate heating or biasing. Following this, selected samples were annealed in an atmospheric environment for $1.5-8 \mathrm{~h}$ at $500{ }^{\circ} \mathrm{C}$ with heating and cooling rates of $2{ }^{\circ} \mathrm{C} \mathrm{min}-1$.

\subsection{2 $\mathrm{TiO}_{2}$ thin film characterization}

X-ray photoelectron spectroscopic (XPS) measurements were performed using Quantera SXM with monochromatic $\mathrm{Al} \mathrm{K \alpha}$ at $1486.6 \mathrm{eV}$ X-ray source. All spectra were shifted to the binding energy of the adventitious $\mathrm{C}$ 1s peak at $284.8 \mathrm{eV}$. The atomic concentration is calculated with the formula $C_{x}=\frac{\frac{I}{S}}{\sum \frac{I}{S}}$ where $\mathrm{I}$ is the peak area and $\mathrm{S}$ the relative sensitivity factor. The intensity of the beam is $2.6 \mathrm{~mA}$ and the beam size is $200 \mu \mathrm{m}$.

The optical properties analysis of the thin films were carried out by a variable 
angle spectroscopic ellipsometer (Woollam M2000-UI) and b-spline model [32].

The crystal structure of the thin films was investigated by XRD (Bruker D2) using CuK- $\alpha$ radiation at $40 \mathrm{kV}$ and $40 \mathrm{~mA}$ working in the $\theta-2 \theta$ mode.

Charge carriers mobilities were investigated using Electrodeless time-resolved microwave conductance (TRMC) technique. (TRMC) technique is based on recording the change of microwave power reflected by a microwave cell on optical excitation of the $\mathrm{TiO}_{2}$ with a short laser pulse. From the normalised change in microwave power the photoconductance can be derived that is related to the product of the yield of photogeneration and the total mobility of the electrons and holes $\left(\mu_{e}+\mu_{h}\right)$. The measurement was conducted using X-band (8.2-12.4 GHz) microwaves $(>100 \mathrm{~mW}$ ), generated by a voltage controlled oscillator (Sivers IMA-Sweden) were carried out at ca $8.4 \mathrm{GHz}$, i.e. the resonant frequency of the loaded cavity. For this measurement the depositions were carried out on quartz substrates due to their excellent transmission properties. A full description of the set-up is given elsewhere [33].

\subsection{Results and Discussion}

\subsection{1 $\mathrm{TiO}_{2}$ Thin film deposition}

As seen in figure 2.1 two different sputtering modes; metallic and oxidized appeared as the oxygen flow rate increased. Up to oxygen flow rate of $4 \mathrm{sccm}$ represents the metallic mode resulting in a sub-stoichiometric film. At higher flowrates, a stoichiometic film is obtained. The abrupt increase in discharge voltage between these two regimes is due to the formation of $\mathrm{TiO}_{2}$ on the target, requiring a higher discharge voltage $[34,35]$.

Two samples, from here on named A and B, which were sputtered at 4 and 5 sccm oxygen flow rate respectively (figure 2.1), were selected and further analysed.

\subsubsection{Optical properties}

The extinction coefficient spectra of A, B and Titanium (Ti), which was sputtered in absence of oxygen, are shown in figure 2.2. The extinction coefficient of 


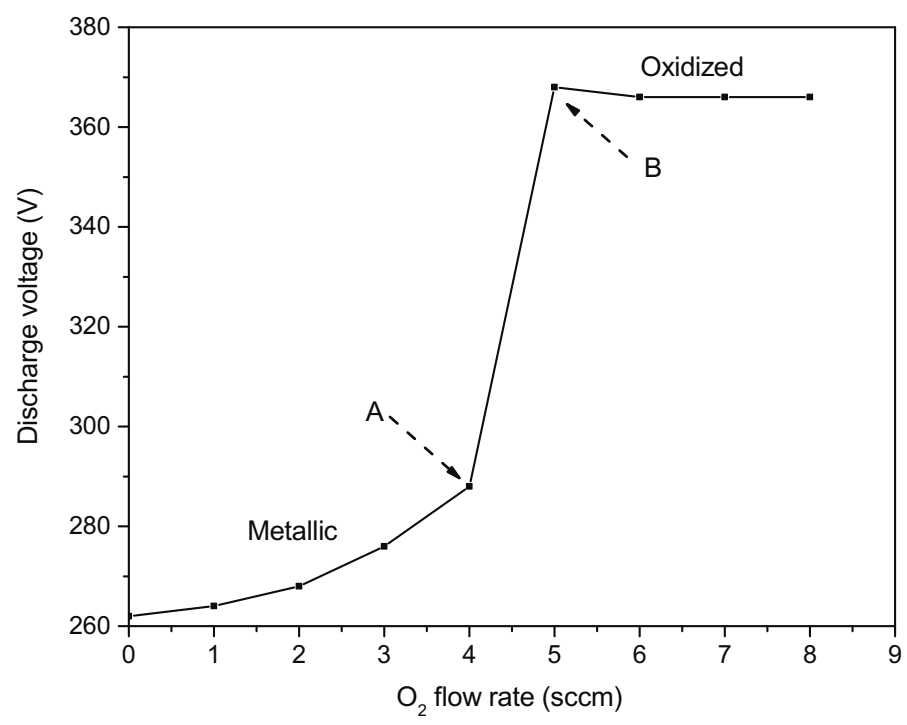

Figure 2.1: Discharge voltage as a function of the oxygen flow rate during reactive magnetron sputtering.

the metallic Ti film is evidently the largest. Film A (4 sccm oxygen flow rate) displays some reminiscence of extinction, while film B (5 sccm oxygen flow rate) is completely transparent in the visible region of the light spectrum. The visible light absorption in film $\mathrm{A}$ is due to the presence of oxygen vacancies [36, 37]. No further differences in terms of extinction coefficient were observed at oxygen flow rates higher than $5 \mathrm{sccm}$.

The in-situ extraction of the refractive index and extinction coefficient changes during annealing of the sub-stoichiometric sample (A) has proven challenging. This is probably due to strong alterations of the sample's optical properties during the process. In particular, a composition gradient in the normal direction as a result of the oxidation reaction develops. To capture this adequately, such a gradient would require grading the B-spline optical model by, for instance, segmenting the sample in several layers with distinct optical properties. This however would introduce a large number of fitting parameters making the procedure less reliable. For similar reasons, attempts to elucidate morphological or structural changes within the sample in the in-situ process proved unreliable. Therefore, in figure 2.3 the dynamic evolution of raw ellipsometry data ( $\psi$ parameter, the amplitude component of the complex reflectance ratio) at 3 different 
wavelengths is shown during annealing in air (from $25^{\circ} \mathrm{C}$ to $500{ }^{\circ} \mathrm{C}$ with $5{ }^{\circ} \mathrm{C} / \mathrm{min}$ ramp rate). The different wavelengths are chosen to represent 3 distinct regions of the sample optical response. At $230 \mathrm{~nm}$ both the as-deposited and annealed samples are absorbing, $365 \mathrm{~nm}$ represents the approximate position of the band gap of the annealed sample, and $800 \mathrm{~nm}$ represents the far visible light. The examination of the $\psi$ dynamics shows that the oxidation onsets at around 150 ${ }^{\circ} \mathrm{C}$ and proceeds to full conversion in about 1 hour after reaching $500{ }^{\circ} \mathrm{C}$, after which it slightly change during the cooling ramp. In particular the large variation in $800 \mathrm{~nm}$ data signify the rapid development of transparency as the oxidation reaction proceeds. The inset shows the resulting extinction coefficients before and after annealing.

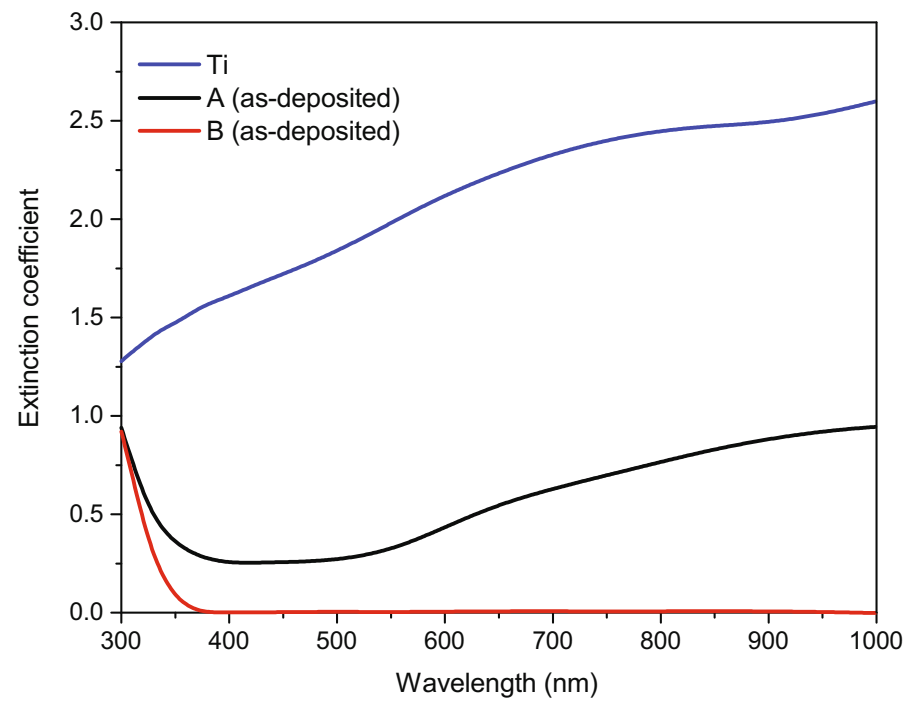

Figure 2.2: Extinction coefficient spectra measured by ellipsometry on a metallic $\mathrm{Ti}$ film, as deposited film (A) sputtered at $4 \mathrm{sccm}$ and as deposited film (B) sputtered at 5 sccm oxygen.

\subsubsection{Structure and chemistry}

Figure 2.4 presents high resolution XPS scans of the Ti $2 \mathrm{p}$ for sample A before and after annealing in air. Sample B, which was sputtered in the oxidized region, matches with $\mathrm{Ti} 2 \mathrm{p}$ scan of stoichiometric $\mathrm{TiO}_{2}$ both before and after annealing [38]. The presence of the shoulder peaks in $\mathrm{Ti} 2 \mathrm{p}\left(\mathrm{Ti}^{3+}\right)$ of the unannealed 


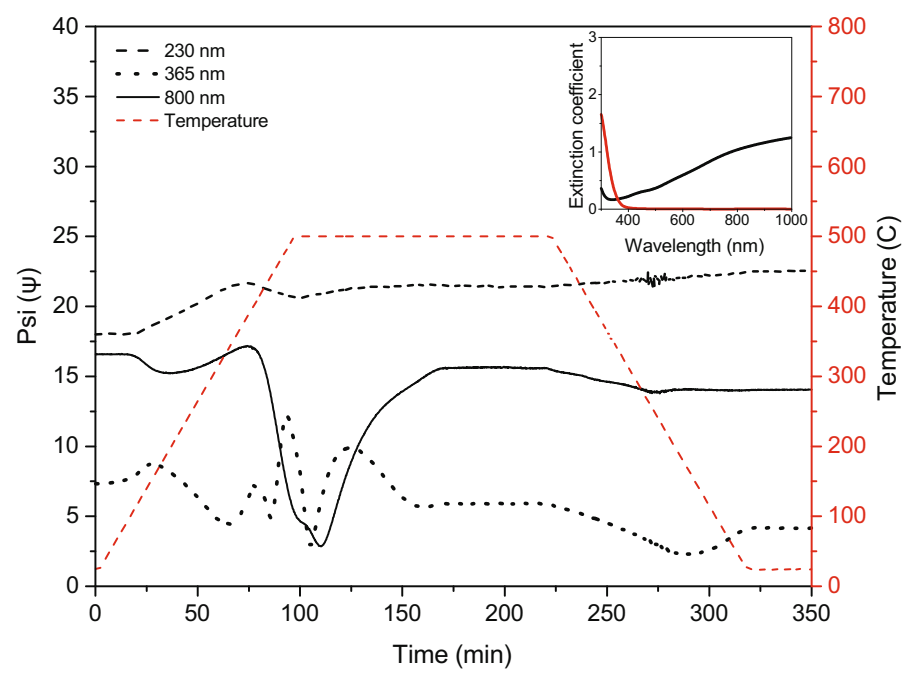

Figure 2.3: Psi $(\psi)$ for film $(\mathrm{A})$ at three different wavelengths during annealing in air with indicated temperature ramp. The inset shows the extinction coefficient before (black) and after (red) annealing in air.

sample A indicate oxygen deficiencies $[38,39]$. The shoulder peaks disappeared following the annealing and matched to sample B suggesting the formation of stoichiometric $\mathrm{TiO}_{2}[38]$.

In addition to the XPS scans of the Ti $2 \mathrm{p}$ and $\mathrm{O}$ 1s core level, the compositional measurement following $4 \mathrm{~nm}$ removal of surface by an Ar gun on sample A reveals $\mathrm{TiO}_{1.8}$ and $\mathrm{TiO}_{2}$ before and after annealing, respectively. It is observed that the extinction coefficient of sample (A) strongly reduces in the visible range after annealing. The film becomes stoichiometric $\mathrm{TiO}_{2}$ with absorption in the UV region of the light spectrum [35].

Figure 2.5 presents the XRD patterns of the as-deposited film, film (A) and film (B) after annealing. What is interesting in this figure is that although the both films are similar in terms extinction coefficient and composition after annealing, film A and B display diffraction peaks that correspond to rutile (110) and anatase (101), respectively [40]. The extracted refractive index of film A and $\mathrm{B}$ after annealing is 2.75 and 2.54, respectively, being in close agreement with reported refractive indices for rutile and anatase phases [41, 42]. The extracted band gaps after annealing are 3.08 and $3.2 \mathrm{ev}$ which also corresponds to the value for the rutile and anatase polymorphs respectively $[43,44]$. 

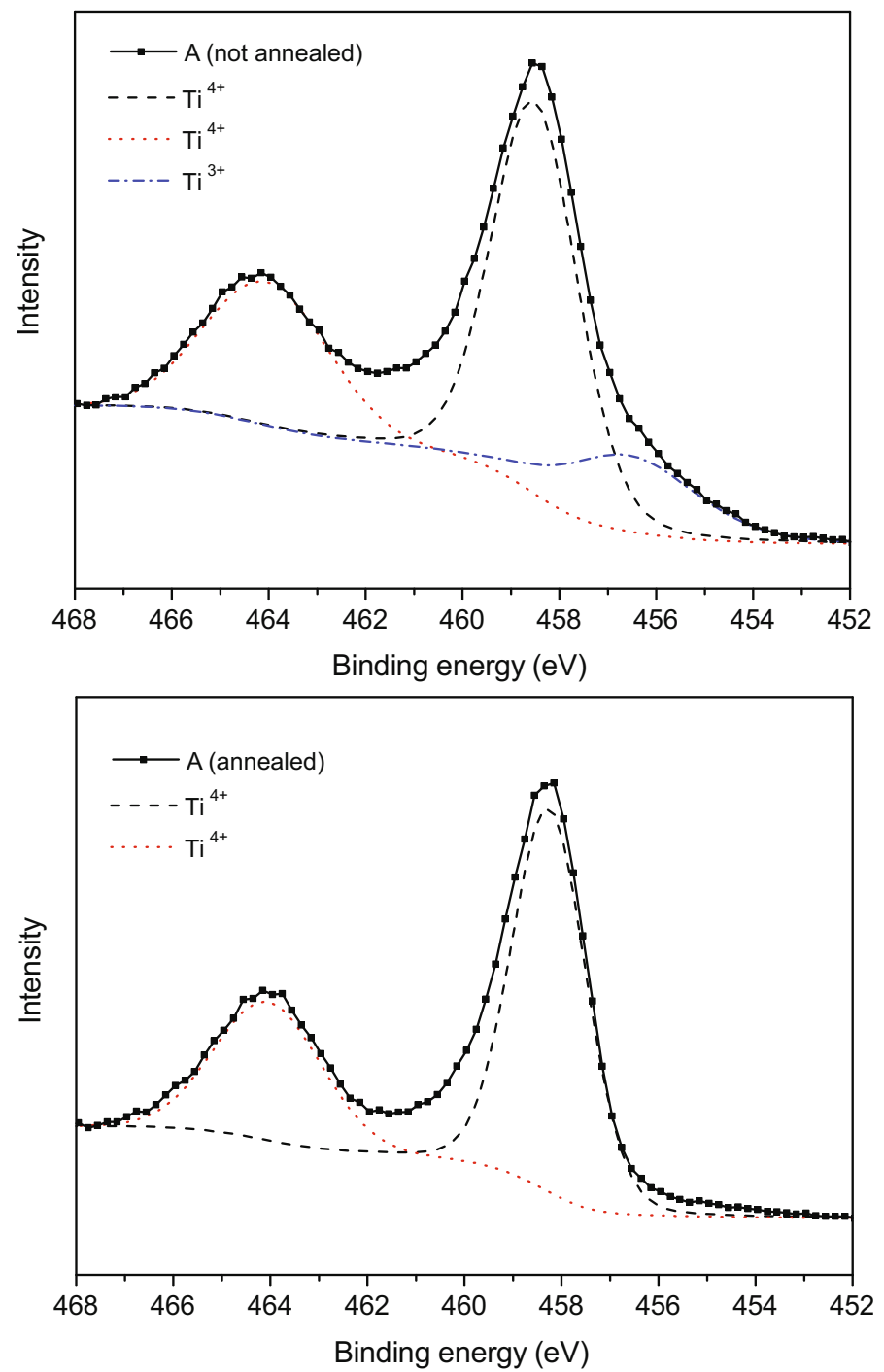

Figure 2.4: High resolution XPS scan of the Ti $2 p$ and $O$ 1s core level of the asdeposited and annealed thin film sputtered at 4 and $5 \mathrm{sccm}$

\subsubsection{Charge carriers mobility}

Figure 2.6 shows the intensity normalized photoconductance transients obtained on pulsed optical excitation at $\lambda=300 \mathrm{~nm}$ for sample A (insert) and B, both after annealing corresponding to rutile and anatase respectively. Since the photon energy used is well above the bandgap of both polymorphs, optical excitation 


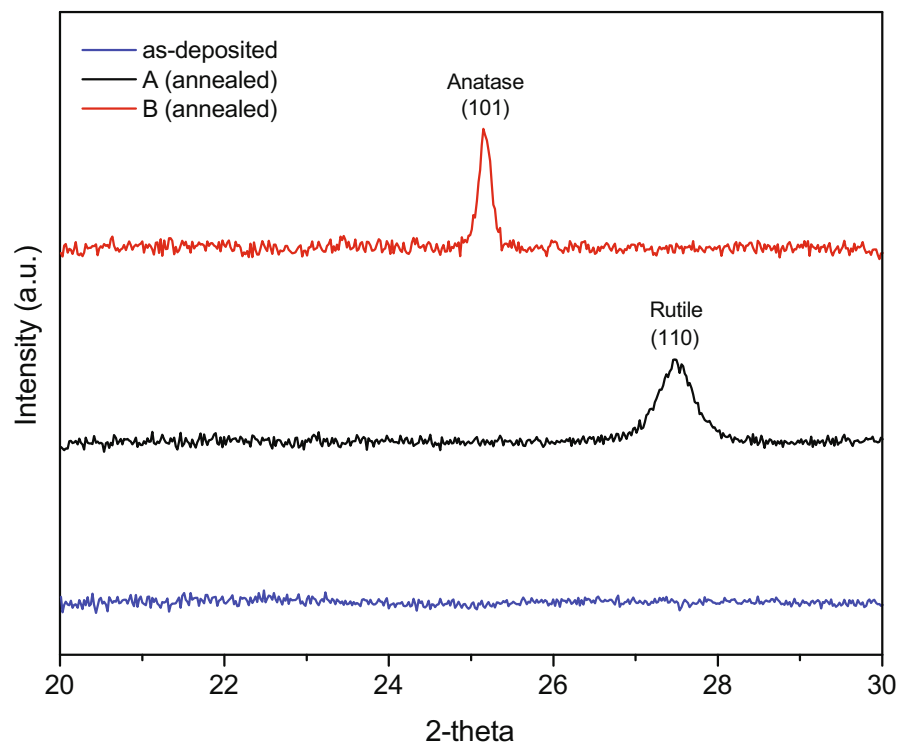

Figure 2.5: X-ray diffraction patterns of the sputtered thin films as-deposited, (A) deposited at 4 and (B) $5 \mathrm{sccm}$ oxygen flow rate after annealing in air.

leads to the formation of mobile carriers resulting in a fast rise of the microwave signal. The decay of the signals is due to immobilization of mobile carriers in trap states or electron hole recombination. The incident laser intensity was varied from $4 \times 10^{12}$ photons $/ \mathrm{cm}^{2}$ to $167 \times 10^{12}$ photons $/ \mathrm{cm}^{2}$ per pulse. It is important to note that although normalized photoconductance transients are shown, the maximum signal size increases first from about $2 \times 10^{-3} \mathrm{~cm}^{2} / \mathrm{Vs}$ to about $25 \times 10^{-3} \mathrm{~cm}^{2} / \mathrm{Vs}$ with increasing intensity.

This has been observed previously for various anatase nanostructured $\mathrm{TiO}_{2}$ and is attributed to trap filling [45-48]. When using even higher laser intensities, the signal decreases again due to the fact that multiple charge carrier pairs are generated per particle leading to rapid sub-nanosecond charge carrier recombination. With higher intensities also the lifetime of the charge carriers reduces. Interestingly, the TRMC signals recorded for sample A (rutile) display a very different photophysical behavior. The maximum signal sizes are more than an order of magnitude smaller which can well be explained by the fact that for rutile the charge carrier mobilities are lower. More importantly, the lifetimes are much smaller (<100 ns) limiting the period the photo-induced carriers are available for 


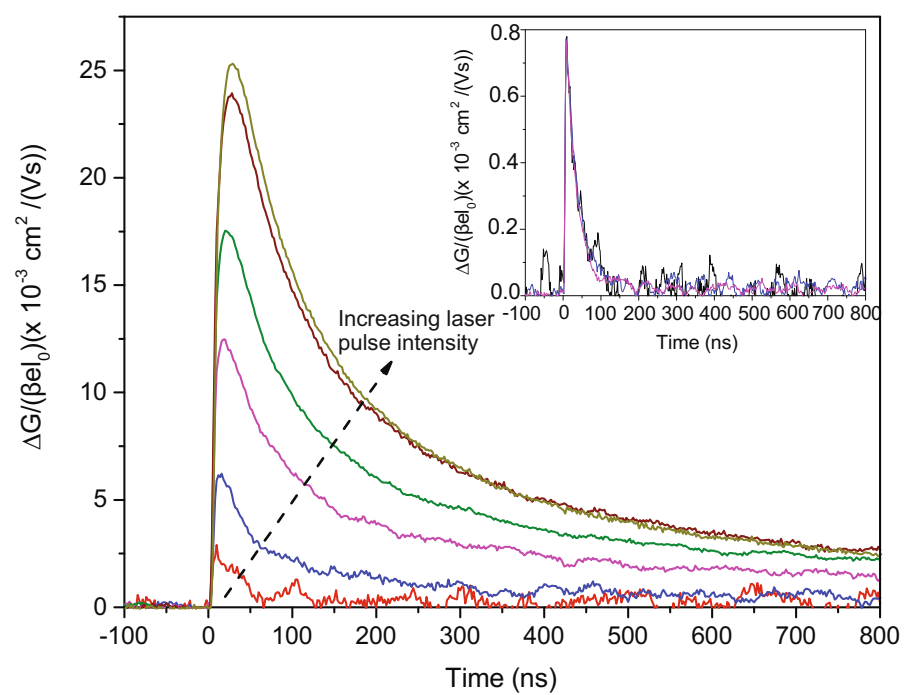

Figure 2.6: Intensity normalised photoconductance transients after excitation by laser pulse at different intensities on sample A (insert) and B after annealing.

consecutive reactions. It is reported that in anatase phase there is surface hole trapping due to the intrinsic surface band bending. Hence the charge separation is enhanced which leads to longer charge carrier life time. On the other hand in rutile phase electrons and holes undergo bulk recombination and only the holes in the vicinity of the surface are trapped and transfer to the surface leading to lower charge carrier life time [49]. All in All is in full agreement with reduced photocatalytic activity found previously for rutile thin films [50]. The anatase thin film demonstrated significantly high photocatalytic activity as reported in our previous study and next chapter [9].

\subsection{Conclusion}

To conclude, our findings provide a methodology for deposition of thin films of $\mathrm{TiO}_{2}$ with selective crystal phase based on the oxygen concentration during reactive magnetron sputtering. Thin films of $\mathrm{TiO}_{2}$ were deposited at low (A) and high (B) oxygen flow rates, resulting in substoichiometric and stoichiometric films respectively. During annealing in air these films correspondingly turn into anatase and rutile, as confirmed by XRD and spectroscopic ellipsometry. The 
anatase film furthermore displayed high photoconductance with longer lifetime charge carriers than rutile. 



\section{Bibliography}

[1] G. Pfaff and P. Reynders, Angle-Dependent Optical Effects Deriving from Submicron Structures of Films and Pigments, Chemical Reviews (Washington, DC, United States) 99, 1963 (1999).

[2] Q. Li, S. Mahendra, D. Y. Lyon, L. Brunet, M. V. Liga, D. Li, and P. J. J. Alvarez, Antimicrobial nanomaterials for water disinfection and microbial control: Potential applications and implications, Water Research 42, 4591 (2008).

[3] R. Dunford, A. Salinaro, L. Cai, N. Serpone, S. Horikoshi, H. Hidaka, and J. Knowland, Chemical oxidation and DNA damage catalysed by inorganic sunscreen ingredients, FEBS Letters 418, 87 (1997).

[4] J. Zhao and X. Yang, Photocatalytic oxidation for indoor air purification: A literature review, Build. Sci. 38, 645 (2003).

[5] K. Fujihara, T. Ohno, and M. Matsumura, Splitting of water by electrochemical combination of two photocatalytic reactions on $\mathrm{TiO}_{2}$ particles, J. Chem. Soc., Faraday Trans. 94, 3705 (1998).

[6] D. Mitoraj, A. Jaczyk, M. Strus, H. Kisch, G. Stochel, P. B. Heczko, and W. Macyk, Visible light inactivation of bacteria and fungi by modified titanium dioxide., Photochemical and Photobiological Sciences 6, 642 (2007).

[7] T. G. Deepak, D. Subash, G. S. Anjusree, K. R. N. Pai, S. V. Nair, and A. S. Nair, Photovoltaic Property of Anatase $\mathrm{TiO}_{2}$ 3-D Meso flowers, ACS Sustainable Chem. Eng. 12, 2772 (2014).

[8] A. Popa, C. Ta, T. Gemming, A. Leonhardt, B. Bu, and R. Klingeler, Anatase Nanotubes as an Electrode Material for Lithium-Ion Batteries, J. Phys. Chem. C 116, 8714 (2012).

[9] D. Rafieian, R. T. Driessen, W. Ogieglo, and R. G. Lammertink, Intrinsic Photocatalytic Assessment of Reactively Sputtered $\mathrm{TiO}_{2}$ Films, ACS Applied 
Materials \& Interfaces 7, 8727 (2015).

[10] V. P. Gupta and N. M. Ravindra, Optoelectronic properties of rutile ( $\left.\mathrm{TiO}_{2}\right)$, Journal of Physics and Chemistry of Solids 41, 591 (1980).

[11] L. F. Arias, A. Kleiman, E. Heredia, and a. Márquez, Rutile titanium dioxide films deposited with a vacuum arc at different temperatures, J. Phys.: Conf. Ser. 370, 012027 (2012).

[12] L.-D. Piveteau, B. Gasser, and L. Schlapbach, Evaluating mechanical adhesion of solgel titanium dioxide coatings containing calcium phosphate for metal implant application, Biomaterials 21, 2193 (2000).

[13] A. Visan, D. Rafieian, W. Ogieglo, and R. G. H. Lammertink, Modeling intrinsic kinetics in immobilized photocatalytic microreactors, Applied Catalysis, B: Environmental 150-151, 93 (2014).

[14] T.-S. Yang, C.-B. Shiu, and M.-S. Wong, Structure and Hydrophilicity of Titanium Oxide Films prepared by Electron Beam Evaporation, Surface Science 548, 75 (2004).

[15] A. Manivannan, N. Spataru, K. Arihara, and A. Fujishima, Electrochemical Deposition of Titanium Oxide on Boron-Doped Diamond Electrodes, Electrochemical and Solid-State Letters 8, C138 (2005).

[16] L. M. Williams, Structural properties of titanium dioxide films deposited in an rf glow discharge, Journal of Vacuum Science \& Technology, A: Vacuum, Surfaces, and Films 1, 1810 (1983).

[17] T. Kubart, J. Jensen, T. Nyberg, L. Liljeholm, D. Depla, and S. Berg, Influence of the target composition on reactively sputtered titanium oxide films, Vacuum 83, 1295 (2009).

[18] Y. Suda, H. Kawasaki, T. Ueda, and T. Ohshima, Preparation of high quality nitrogen doped $\mathrm{TiO}_{2}$ thin film as a photocatalyst using a pulsed laser deposition method, Thin Solid Films 453-454, 162 (2004).

[19] S. A. O’Neill, I. P. Parkin, R. J. H. Clark, A. Mills, and N. Elliott, Atmospheric pressure chemical vapour deposition of titanium dioxide coatings on glass, Journal of Materials Chemistry 13, 56 (2003).

[20] J. Aarik, A. Aidla, H. Mändar, and T. Uustare, Atomic Layer Deposition of Titanium Dioxide from $\mathrm{TiCl}_{4}$ and $\mathrm{H}_{2} \mathrm{O}$ : Investigation of Growth Mechanism, Applied Surface Science 172, 148 (2001). 
[21] H. Shin, H. S. Jung, K. S. Hong, and J.-K. Lee, Crystal phase evolution of $\mathrm{TiO}_{2}$ nanoparticles with reaction time in acidic solutions studied via freezedrying method, Journal of Solid State Chemistry 178, 15 (2005).

[22] S. Meyer, R. Gorges, and G. Kreisel, Preparation and characterisation of titanium dioxide films for catalytic applications generated by anodic spark deposition, Thin Solid Films 450, 276 (2004).

[23] C. Rath, P. Mohanty, A. C. Pandey, and N. C. Mishra, Nanoparticles, Journal of Physics D: Applied Physics 42, 205101 (2009).

[24] T. B. Ghosh, Erratum: On crystalline size dependence of phase stability of nanocrystalline $\mathrm{TiO}_{2}$, Journal of Applied Physics 95, 408 (2004).

[25] M. Hirano, C. Nakahara, K. Ota, O. Tanaike, and M. Inagaki, Photoactivity and phase stability of $\mathrm{ZrO}_{2}$-doped anatase-type $\mathrm{TiO}_{2}$ directly formed as nanometer-sized particles by hydrolysis under hydrothermal conditions, Journal of Solid State Chemistry 170, 39 (2003).

[26] G. Li, L. Li, J. Boerio-Goates, and B. F. Woodfield, High purity anatase $\mathrm{TiO}_{2}$ nanocrystals: Near room-temperature synthesis, grain growth kinetics, and surface hydration chemistry, Journal of the American Chemical Society 127, 8659 (2005).

[27] D. Yoo, I. Kim, S. Kim, C. H. Hahn, C. Lee, and S. Cho, Effects of annealing temperature and method on structural and optical properties of $\mathrm{TiO}_{2}$ films prepared by RF magnetron sputtering at room temperature, Applied Surface Science 253, 3888 (2007).

[28] B. Choudhury and A. Choudhury, Local structure modification and phase transformation of $\mathrm{TiO}_{2}$ nanoparticles initiated by oxygen defects, grain size, and annealing temperature, Int. Nano Lett. 3, 55 (2013).

[29] D. Wicaksana, T. Tsujikawa, A. Kobayashi, K. Ono, and A. Kinbara, Ion bombardment effects on the growth of rutile phase of reactively sputtered $\mathrm{TiO}_{2}$ thin films (1993), Vol. 311, pp. 233-238, cited By 6.

[30] S. S. Pradhan, S. K. Pradhan, V. Bhavanasi, S. Sahoo, S. N. Sarangi, S. Anwar, and P. K. Barhai, Low temperature stabilized rutile phase $\mathrm{TiO}_{2}$ films grown by sputtering, Thin Solid Films 520, 1809 (2012).

[31] H. A. Shukur, M. Sato, I. Nakamura, and I. Takano, Characteristics and photocatalytic properties of $\mathrm{TiO}_{2}$ thin film prepared by sputter deposition 
and post- $N+$ ion implantation, Adv. Mater. Sci. Eng. 2012, 7 (2012).

[32] B. Johs and J. S. Hale, Dielectric Function representation by B-splines, Physica Status Solidi A: Applications and Materials Science 205, 715 (2008).

[33] T. J. Savenije, A. J. Ferguson, N. Kopidakis, and G. Rumbles, Revealing the Dynamics of Charge Carriers in Polymer:Fullerene Blends Using Photoinduced Time-Resolved Microwave Conductivity, J. Phys. Chem. C 117, 24085 (2013).

[34] D. Depla, S. Heirwegh, S. Mahieu, J. Haemers, and R. De Gryse, Understanding the discharge voltage behavior during reactive sputtering of oxides, Journal of Applied Physics 101, 013301 (2007).

[35] C. J. Tavares, J. Vieira, L. Rebouta, G. Hungerford, P. Coutinho, V. Teixeira, J. O. Carneiro, and A. J. Fernandes, Reactive sputtering deposition of photocatalytic $\mathrm{TiO}_{2}$ thin films on glass substrates, J. Mater. Sci. Eng. B 138, 139 (2007).

[36] C. Guillén, J. Montero, and J. Herrero, Anatase andrutile $\mathrm{TiO}_{2}$ thin films prepared by reactive DC sputtering at high deposition rates on glass and flexible polyimide substrates, Journal of Materials Science 49, 5035 (2014).

[37] R. Pandian, G. Natarajan, S. Rajagopalan, M. Kamruddin, and A. K. Tyagi, On the phase formation of titanium oxide thin films deposited by reactive DC magnetron sputtering: influence of oxygen partial pressure and nitrogen doping, Applied Physics A: Materials Science \& Processing 116, 1905 (2014).

[38] T. Hanawa, A comprehensive review of techniques for biofunctionalization of titanium, J. Periodontal Implant Sci. 41, 263 (2011).

[39] K. Zakrzewska, Nonstoichiometry in $\mathrm{TiO}_{2-y}$ studied by ion beam methods and photoelectron spectroscopy, Advances in Materials Science and Engineering 2012, 13 (2012).

[40] A. Fujishima, X. Zhang, and D. A. Tryk, Surface Science Reports TiO 2 photocatalysis and related surface phenomena, Surface Science Reports 63, 515 (2008).

[41] D. a. H. Hanaor and C. C. Sorrell, Review of the anatase to rutile phase transformation, Journal of Materials Science 46, 855 (2010).

[42] T. Jones and T. A. Egerton, Titanium compounds, inorganic (Wiley, 2000), No. 3757, pp. 1637-1638. 
[43] A. Beltran, L. Gracia, and J. Andres, Density functional theory study of the brookite surfaces and phase transitions between natural titania polymorphs, Journal of Physical Chemistry B 110, 23417 (2006).

[44] D. Mardare, M. Tasca, M. Delibas, and G. I. Rusu, On the structural properties and optical transmittance of $\mathrm{TiO}_{2}$ r.f. sputtered thin films, Applied Surface Science 156, 200 (2000).

[45] J. E. Kroeze, T. J. Savenije, and J. M. Warman, Electrodeless determination of the trap density, decay kinetics, and charge separation efficiency of dyesensitized nanocrystalline $\mathrm{TiO}_{2}$, Journal of the American Chemical Society 126, 7608 (2004).

[46] T. J. Savenije, A. Huijser, M. J. W. Vermeulen, and R. Katoh, Charge carrier dynamics in $\mathrm{TiO}_{2}$ nanoparticles at various temperatures, Chemical Physics Letters 461, 93 (2008).

[47] J. T. Carneiro, T. J. Savenije, and G. Mul, Experimental evidence for electron localization on Au upon photo-activation of Au/anatase catalysts., Physical Chemistry Chemical Physics 11, 2708 (2009).

[48] M. C. Fravventura, D. Deligiannis, J. M. Schins, L. D. a. Siebbeles, and T. J. Savenije, What limits photoconductance in anatase $\mathrm{TiO}_{2}$ nanostructures? A real and imaginary microwave conductance study, J. Phys. Chem. C 117, 8032 (2013).

[49] G. Li, L. Chen, M. E. Graham, and K. a. Gray, A comparison of mixed phase titania photocatalysts prepared by physical and chemical methods: The importance of the solidsolid interface, Journal of Molecular Catalysis A: Chemical 275, 30 (2007).

[50] T. Luttrell, S. Halpegamage, J. Tao, A. Kramer, E. Sutter, and M. Batzill, Why is anatase a better photocatalyst than rutile?-Model studies on epitaxial $\mathrm{TiO}_{2}$ films., Sci. Rep. 4, 4043 (2014). 



\section{CHAPTER 3}

\section{Intrinsic Photocatalytic Assessment of Reactively}

\section{Sputtered $\mathrm{TiO}_{2}$ Films}

Thin $\mathrm{TiO}_{2}$ films were prepared by $\mathrm{DC}$ magnetron reactive sputtering at different oxygen partial pressures. Depending on the oxygen partial pressure during sputtering, a transition from metallic $\mathrm{Ti}$ to $\mathrm{TiO}_{2}$ was identified by spectroscopic ellipsometry. The crystalline nature of the film developed during a subsequent annealing step, resulting in thin anatase $\mathrm{TiO}_{2}$ layers, displaying photocatalytic activity. The intrinsic photocatalytic activity of the catalysts was evaluated for the degradation of methylene blue using a microfluidic reactor. A numerical model was employed to extract the intrinsic reaction rate constants. High conversion rates (90\% degradation within $20 \mathrm{~s}$ residence time) were observed within these microreactors due to the efficient mass transport and light distribution. To evaluate the intrinsic reaction kinetics, we argue that mass transport has to be accounted for. The obtained surface reaction rate constants demonstrate very high reactivity for the sputtered $\mathrm{TiO}_{2}$ films. Only for the thinnest film, $9 \mathrm{~nm}$, slightly lower kinetics were observed.

This chapter has been published as: Damon Rafieian, Rick T. Driessen, Wojciech Ogieglo and Rob G.H. Lammertink, Intrinsic Photocatalytic Assessment of Reactively Sputtered $\mathrm{TiO}_{2}$ Films, ACS Applied Materials \& Interfaces 20157 (16), 8727-8732, DOI: 10.1021/acsami.5b01047. 


\subsection{Introduction}

Heterogeneous photocatalysis using semiconductors finds increasing interest related to self-cleaning, water purification and air cleaning applications [1, 2]. From many semiconductors, $\mathrm{TiO}_{2}$ is the most studied one regarding photocatalysis. Light with energy equal or higher than the band gap energy of $\mathrm{TiO}_{2}$ leads to the formation of electron-hole pairs. The generated electron-hole pairs can in turn decompose organic contaminants via diverse reaction schemes. $\mathrm{TiO}_{2}$ has three well-known crystal structures; anatase, rutile and brookite. The photocatalytic performance of the anatase and rutile structures has been subjected to extensive research $[3,4]$. The anatase phase has a band gap of $3.2 \mathrm{eV}$ which corresponds to a wavelength of $385 \mathrm{~nm}[1,4]$. The anatase phase is formed at temperatures below $400-600{ }^{\circ} \mathrm{C}$ while above $800{ }^{\circ} \mathrm{C}$ the rutile phase appears [5]. Furthermore, the formation of smaller grains at lower temperature leads to better photocatalytic activity. For grains below $10 \mathrm{~nm}$, however, boundaries affect the photocatalytic activity unfavorably by increasing the recombination rate. This explains the reduced photocatalytic activity observed in amorphous $\mathrm{TiO}_{2}[6]$.

Titanium dioxide thin films can be synthesized by techniques such as sol-gel [7], suspension coating [8], electron beam evaporation [9], electrochemical deposition [10], different sputtering configurations [11, 12], pulsed laser deposition (PLD) [13] and many other methods $[14,15]$. Among them, reactive magnetron sputtering is of paramount interest since the morphology, composition and crystallinity can be accurately controlled by modifying the deposition conditions. The resulting $\mathrm{TiO}_{2}$ thin films present high uniformity over large areas which makes them attractive for both applications and fundamental studies as well. Hence there is a rapidly growing literature on utilizing sputtered $\mathrm{TiO}_{2}$ thin films for photocatalytic studies [16-18]. Mostly, the main focus is directed toward the structure of the thin film as a result of different process variables and sputtering configurations $[6]$.

It is remarkable to observe that throughout literature, many photocatalytic degradations using sputtered $\mathrm{TiO}_{2}$ films are characterized by just presenting the concentration versus time[19, 20] or assuming first order bulk reaction kinetics [21-24]. The latter approach relates $\ln \left(c_{0} / c\right)$ to the reaction time, to obtain the 
apparent (volumetric) reaction rate constant which is then expressed in $s^{-1}$. In order to obtain the apparent surface reaction rate constant, one should multiply this bulk reaction rate constant $k$ with the reactor volume to catalyst surface area ratio. Here, we will argue that besides the introduction of the volume/area ratio, mass transport has to be accounted for when evaluating the photocatalytic activity of immobilized $\mathrm{TiO}_{2}$ thin films. The degradation of methylene blue (MB) is analyzed using dense and thin $\mathrm{TiO}_{2}$ films within a microfluidic reactor, where we account for mass transport. By quantifying the reaction and diffusion rates in terms of the second Damköhler number, we demonstrate the fast photocatalytic kinetics and consequently the need for mass transport inclusion.

\subsection{Materials and methods}

\subsubsection{Photocatalyst Synthesis}

Silicon wafer p-type (100) as the substrate was cleaned in $98 \%$ nitric acid for 10 minutes and followed by rinsing and drying prior to deposition. $\mathrm{TiO}_{2}$ thin films were prepared using a custom made DC magnetron sputtering system (TCOATER, MESA+ Nanolab) in cleanroom environment. A $10 \mathrm{~cm}$ diameter titanium disk of $99.7 \%$ purity was used as the deposition target. The substratetarget distance was set at $4.4 \mathrm{~cm}$ and the substrate rotated at $5 \mathrm{rpm}$ during the whole deposition process for enhanced uniformity. The sputtering chamber was evacuated to a base pressure of $10^{-7}$ mbar at room temperature prior to the deposition. A mixture of $99.5 \%$ pure oxygen and $99.99 \%$ pure argon were used as the sputtering and reactive gas respectively. The total process pressure was regulated at $6 \times 10^{-3}$ mbar in all the experiments. The target was powered by a variable DC-power supply at $500 \mathrm{~W}$ and pre-sputtered for 2 minutes with a shutter covering the substrates in all experimental trials. No extra heating and biasing were applied to substrates in all of the depositions steps. After the sputtering, the silicon wafer was diced into $24 \times 17 \mathrm{~mm}$ chips (Dicing saw Disco DAD 321). Some chips were annealed in an atmospheric environment for $8 \mathrm{~h}$ at 500 ${ }^{\circ} \mathrm{C}$ with heating and cooling rates of $2{ }^{\circ} \mathrm{C} \mathrm{min}^{-1}$. 


\subsubsection{Catalyst layer characterization}

The morphology of the thin films was analyzed by high resolution scanning electron microscopy (Analysis Zeiss MERLIN HR-SEM). Thickness measurements were carried out by a variable angle spectroscopic ellipsometer (Woollam M2000UI) and surface profiler (Bruker Dektak 150). The ellipsometry data was analyzed using the b-spline model to extract optical properties of the thin films [25]. The crystal structure of the thin films was investigated by XRD (Bruker D2) using CuK- $\alpha$ radiation at $40 \mathrm{kV}$ and $40 \mathrm{~mA}$ working in the $\theta-2 \theta$ mode.

\subsubsection{Microreactor fabrication}

A microstructured mold was made by standard photolithography using negative photoresist (SU-8) in cleanroom environment. SU-8 was spun on silicon wafer at $500 \mathrm{rpm}$ for 10 seconds and continued to $100 \mathrm{rpm}$ for 30 seconds. Then the sample was pre-baked at 50,65 , and $95^{\circ} \mathrm{C}$ for 10,10 , and 45 minutes, respectively. This was followed by UV exposure for 33 seconds and post baking at 50,65 , and $80{ }^{\circ} \mathrm{C}$ for 5, 10, and 20 minutes, respectively. Finally the sample was spray developed by RER 600 . The SU-8 height was measured by an optical profiler (Bruker WLI Contour GT-I). Polydimethylsiloxane (PDMS) was prepared by mixing the polymer base (Permacol RTV-615 A) with the curing agent (Permacol RTV$615 \mathrm{~B})$ in a 10:1 mass ratio, followed by degassing in a dessicator. The PDMS was cast on the mold and further degassed in a dessicator and cured at $60{ }^{\circ} \mathrm{C}$ for 45 minutes. The PDMS slab was removed from the mold and inlets and outlets were punctured. Subsequently, it was attached to the $\mathrm{TiO}_{2}$ substrate. To ensure a leakfree assembly, additional PDMS was slightly pre-cured and poured at the edge of the previously cured PDMS cap and further cured overnight. The fabrication steps are schematically illustrated in Figure 4.2. The channel has a rectangular cross section with basic dimensions of $50 \mu \mathrm{m}$ height, $500 \mu \mathrm{m}$ width and $5.96 \mathrm{~cm}$ length, giving a total volume of $1.49 \mu \mathrm{L}$.

\subsubsection{Microreactor operation}

An aqueous solution of $40 \mu \mathrm{M}$ methylene blue (MB) was injected into the microreactor with flow rates between 3 and $50 \mu \mathrm{L} \mathrm{min} \operatorname{mos}^{-1}$ using a syringe pump 

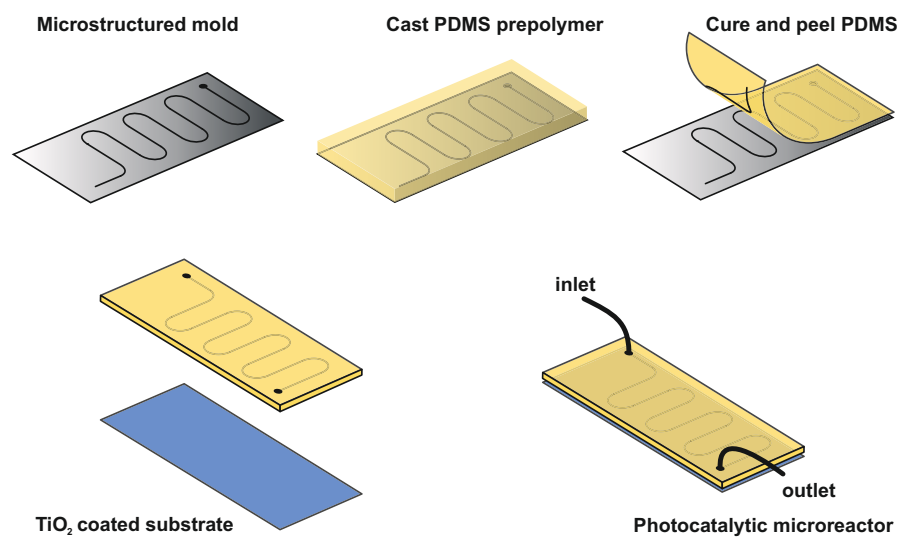

Figure 3.1: Illustration of the microreactor fabrication. The flow channel is defined in PDMS which is subsequently connected to the photocatalytic surface.

(New Era NE-4000). The MB concentration at the outlet was determined by light absorbance measurements using an in-line UV-vis spectrometer (USB2000+ Miniature Fiber Optic Ocean Optics). The monitored wavelength was $664 \mathrm{~nm}$, corresponding to the maximum absorption peak of MB. The calibration was carried out for different concentrations of MB used to convert the observed absorbance to concentration. The degradation experiments were carried our using a UV light source (HP-120 Opystec Dr. Gröbel) fixed at a distance of $4 \mathrm{~cm}$ from the catalyst surface providing $100 \mathrm{~mW} / \mathrm{cm}^{2}$ photon flux density at $365 \mathrm{~nm}$ wavelength.

\subsection{Model}

To establish a fair comparison between different catalysts, the activity assessment is based on obtaining intrinsic reaction rate constants. Here we present a model that describes convection and diffusion for the flow channel combined with a surface reaction term as a boundary condition. The inclusion of the diffusion term makes sure that mass transport limitations are captured as well. The intrinsic reaction rate constant per unit surface area of the catalyst can be extracted by fitting the numerical model to the experimental data.

The 2D model of the microchannel is schematically summarized in Figure 3.2. The assumptions for this model are: (a) the flow is steady, laminar and 


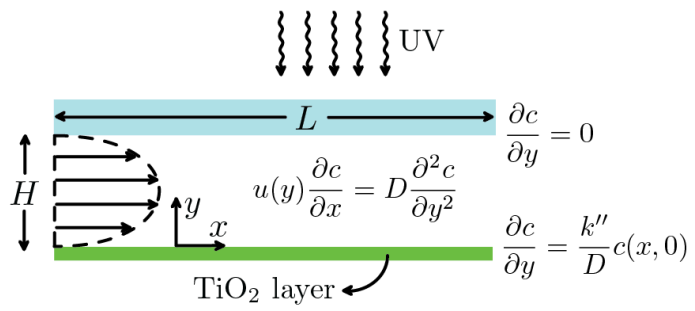

Figure 3.2: Representation of the 2D convection diffusion model. Convection is governed by the parabolic flow profile $u(y)$, while diffusion is only accounted for in the $y$ direction. A first order surface reaction rate is included as the lower boundary condition.

incompressible, (b) the diffusion coefficient is independent of the concentration and $(\mathrm{c})$ thermal effects are neglected.

The mass transport of the model compound in the flow channel is determined by advection (only in $x$ direction) and diffusion (only in $y$ direction):

$$
u(y) \frac{\partial c}{\partial x}=D \frac{\partial^{2} c}{\partial y^{2}}
$$

In this equation $u(y)$ is the fluid velocity, $c(x, y)$ is the reactant concentration and $D$ is the molar diffusion coefficient of the model compound in water. The flow is laminar (as $10^{-4} \leq R e \leq 10^{-3}$ ) hence a parabolic velocity profile can be applied:

$$
u(y)=u_{\text {avg }}\left(\frac{-6 y^{2}}{H^{2}}+\frac{6 y}{H}\right)
$$

The parabolic velocity profile is obtained by solving the Navier-Stokes equation for two parallel flat plates in 2D with laminar flow for the coordinate system shown in Figure 3.2. As our physical device contains channels with a width to height ratio of 10 , this is a fair approximation.

At $x=0$ the concentration is equal to the inlet concentration $c_{0}$ for all $y$ :

$$
\left.c\right|_{x=0}=c_{0}
$$

The upper wall $(y=H)$ is considered impermeable, represented by the Neu- 
mann boundary condition:

$$
\left.\frac{\partial c}{\partial y}\right|_{y=H}=0
$$

For the boundary condition at the lower wall $(y=0)$, the molar flux to the catalyst surface is equal to the rate of consumption of the model compound. Assuming a first-order reaction, the boundary condition reads:

$$
\left.D \frac{\partial c}{\partial y}\right|_{y=0}=\left.k " c\right|_{y=0}
$$

where $k "$ is the intrinsic surface reaction rate constant $\left(\mathrm{m} \mathrm{s}^{-1}\right) . k$ " relates to the 'bulk' $k\left(\mathrm{~s}^{-1}\right)$ constant by taking the reactor volume to catalyst surface into account: $k "=k \frac{V}{A}$.

The equations for the flow channel (3.1 and 3.2) and the boundary conditions (3.4 and 3.5) are non-dimensionalized to yield:

$$
\begin{gathered}
u_{\mathrm{avg}}\left(-6 \tilde{y}^{2}+6 \tilde{y}\right) \frac{H^{2}}{L D} \frac{\partial \tilde{c}}{\partial \tilde{x}}=\frac{\partial^{2} \tilde{c}}{\partial \tilde{y}^{2}} \\
\left.\tilde{c}\right|_{\tilde{x}=0}=1 \\
\left.\frac{\partial \tilde{c}}{\partial \tilde{y}}\right|_{\tilde{y}=1}=0 \\
\left.\frac{\partial \tilde{c}}{\partial \tilde{y}}\right|_{\tilde{y}=0}=\frac{k " H}{D} \tilde{c}=D a_{\mathrm{II}} \tilde{c}
\end{gathered}
$$

where $\tilde{c}=c / c_{0}, \tilde{x}=x / L$, and $\tilde{y}=y / H . D a_{\mathrm{II}}$ is the second Damköhler number defined as the ratio between the chemical reaction rate and the diffusive mass transport [26]. The model is solved numerically in MATLAB using the pdepe solver. The mesh was refined until mesh-independent solutions were obtained. The mixing cup concentration of the outflow was matched to the experimental value, while only $k$ " was varied. 


\subsection{Results and Discussion}

\subsubsection{Film deposition and characterization}

Figure 3.3 (a) shows the sputtering rate as a function of oxygen partial pressure at constant total pressure $\left(6 \times 10^{-3} \mathrm{mbar}\right)$. There is a sharp drop in the sputtering rate at an oxygen partial pressure of $8.57 \times 10^{-4}$ mbar. This transition in sputtering rate typically indicates that the deposited material transits from a metallic to an oxidised form. We extract the extinction coefficient of the deposited thin films at different oxygen partial pressures.
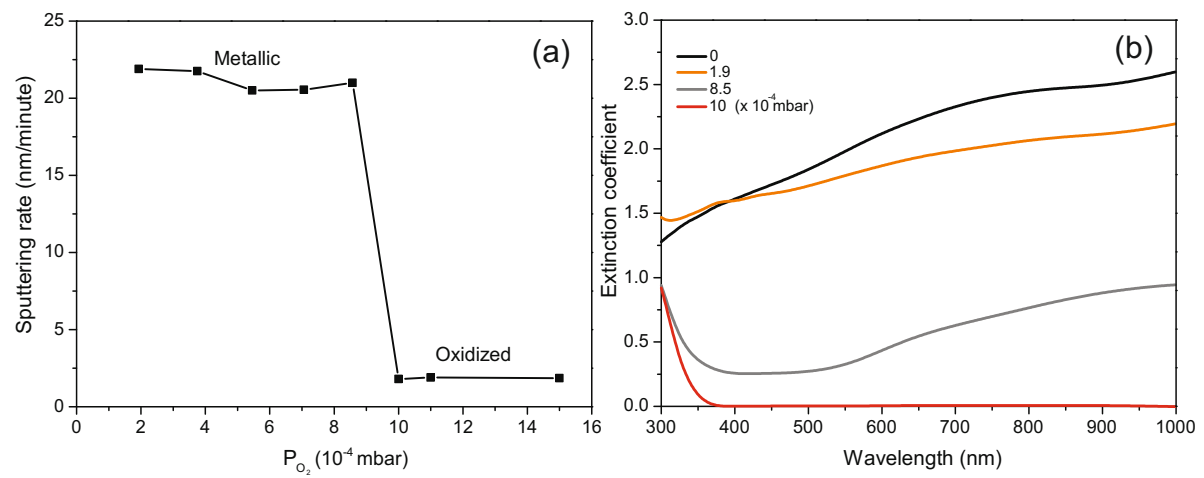

Figure 3.3: (a) Sputtering deposition rate and (b) extinction coefficient of thin films sputtered at different oxygen partial pressures.

As observed in Figure 3.3(b) the extinction coefficient of the deposited films is the highest for the pure titanium and it gradually decreases until complete transparency in the visible region for the film prepared at $10^{-3} \mathrm{mbar}$. It is exactly at this oxygen partial pressure that the stoichiometric $\mathrm{TiO}_{2}$ is formed with absorption in the UV region of the light spectrum [27]. No further differences in terms of extinction coefficient were observed at higher oxygen partial pressures. Figure 3.4 depicts the effect of annealing on the surface morphology of the $\mathrm{TiO}_{2}$ thin film deposited at $10^{-3}$ mbar. The insets display the corresponding X-ray diffraction patterns. The as-deposited film at room temperature is amorphous due to the lack of energy for mobilization of the nucleated particles and formation of crystals with a regular lattice [27]. After annealing, grains are formed 

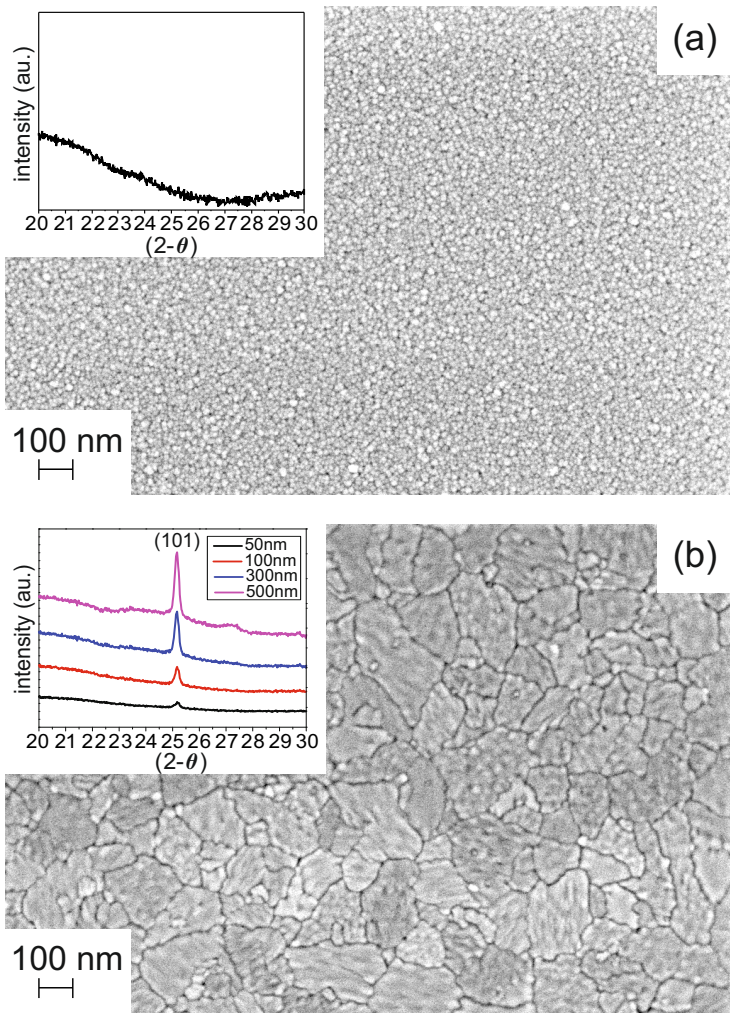

Figure 3.4: The microstructure and $\mathrm{XRD}$ patterns of $\mathrm{TiO}_{2}$ thin film before (a) and after annealing at $500{ }^{\circ} \mathrm{C}$ for 8 hours (b). The assigned peak to the (101) plane is present in all of the film thicknesses.

and the diffraction peak for 101 (anatase) appears. Despite the crystallization during annealing, no significant differences were observed in terms of extinction coefficient. The refractive index of the thin film only increased slightly upon annealing. According to Horprathum et al. this is caused by the reaction between amorphous $\mathrm{TiO}_{2}$ with previously diffused oxygen and densification of the film $[28]$.

\subsubsection{Photocatalytic performance}

Despite the comparable extinction coefficients for $\mathrm{TiO}_{2}$ thin films before and after annealing, the photocatalytic activity differs tremendously. Amorphous thin films display no photocatalytic activity. Possibly, lattice defects generate 
trap sites for the photogenerated electron-holes which increase the recombination rate [6]. Figure 3.5 shows the degradation of $\mathrm{MB}(40 \mu \mathrm{M})$ in the microreactor as a function of residence times utilizing the catalyst sputtered at $10^{-3}$ mbar oxygen partial pressure followed by annealing (Figure 3.4(b)). The concentration of MB was determined by UV-Vis absorbance for different residence times (Figure 3.5 insert). The degradation is calculated by the following equation:

$$
\text { degradation }=\frac{c_{0}-c_{\mathrm{f}}}{c_{0}} \times 100 \%
$$

where $c_{0}$ and $c_{\mathrm{f}}$ are the inlet and outlet concentrations, respectively. The fitted line corresponds to the results of the numerical model. Note that only the surface reaction rate constant $k "$ is adjusted to fit the experimental data, and that mass transport is accounted for by the convection diffusion equation. The standard deviation in degradation is based on the standard deviation of the initial and final concentration. At larger residence times, this standard deviation increases because of the low measured concentration and the long time required to reach steady state. The fitted reaction rate constant $k$ is $0.22 \mathrm{~s}^{-1}\left(k "=1.1 \times 10^{-5} \mathrm{~m}\right.$ $\left.\mathrm{s}^{-1}\right)$ based on the molecular diffusion of MB in water $\left(D=5.7 \times 10^{-10} \mathrm{~m}^{2} \mathrm{~s}^{-1}\right)$ $[8]$.

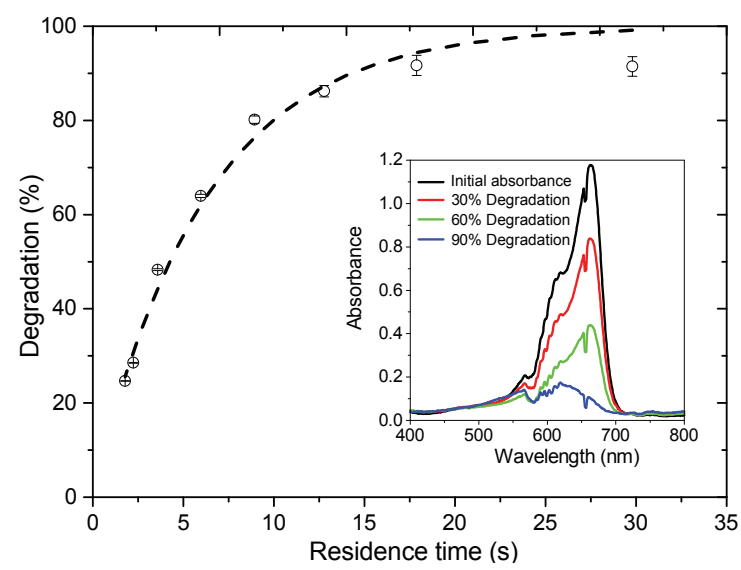

Figure 3.5: Degradation of methylene blue $(40 \mu \mathrm{M})$ as a function of residence time. The fit results from the numerical model using a single fitted surface reaction rate constant $\left(k "=1.1 \times 10^{-5} \mathrm{~m} \mathrm{~s}^{-1}\right)$. The insert shows the initial absorbance spectra (black) and at 30\% (red), 60\% (green) and 90\% (blue) degradation for methylene blue. 
Table 3.1: Overall $(k)$ and surface normalized $(k ")$ photocatalytic reaction rate constant of sputtered $\mathrm{TiO}_{2}$.

\begin{tabular}{lllll}
\hline Sputtering deposition & $k\left(\mathrm{~s}^{-1}\right)$ & $k \prime\left(\mathrm{m} \mathrm{s}^{-1}\right)$ & $\frac{\text { Volume }}{\text { Area }}(\mathrm{m})$ & Ref \\
\hline RF magnetron & $3.09 \times 10^{-5}$ & $4.96 \times 10^{-7}$ & $1.60 \times 10^{-2}$ & {$[24]$} \\
RF magnetron & $1.92 \times 10^{-4}$ & $2.87 \times 10^{-6}$ & $1.5 \times 10^{-2}$ & {$[20]$} \\
Pulsed DC & $2.83 \times 10^{-5}$ & $1.89 \times 10^{-6}$ & $6.67 \times 10^{-2}$ & {$[23]$} \\
Unbalanced DC & $1.99 \times 10^{-5}$ & $7.99 \times 10^{-8}$ & $2.5 \times 10^{-3}$ & {$[19]$} \\
RF magnetron & $1.58 \times 10^{-7}$ & $4.22 \times 10^{-8}$ & $2.67 \times 10^{-1}$ & {$[22]$} \\
DC magnetron & $2.2 \times 10^{-1}$ & $1.1 \times 10^{-5}$ & $5.0 \times 10^{-5}$ & This work \\
\hline
\end{tabular}

Table 3.1 represents the photocatalytic reaction rate constants of reactively sputtered $\mathrm{TiO}_{2}$ films reported in literature and our findings. Throughout literature, photocatalytic performance is most frequently quantified by the apparent reaction rate constant, i.e. by determining the slope of $\ln \frac{c_{0}}{c_{f}}$ versus $t$. This reaction rate constant represents the volume based conversion and does not take the catalyst area per reactor volume into account. It is therefore not suitable to compare catalysts when these have been assessed with different reactor configurations. A better comparison involves the presentation of a surface normalized reaction rate, obtained by multiplying the 'bulk' rate constant $k$ with the reactor volume to catalyst area ratio. In our presented case, this concerns the height of the microchannel $H$.

The extracted surface reaction rate constant for our sputtered film is at least an order of magnitude larger compared to previously reported values. Besides the possible deviations in active surface area and illumination intensities, mass transport limitations can be responsible for the observed difference. Below, we will address the significance of mass transport further, by discussing the second Damköhler number. The extracted rate constant per unit surface area $(k "=$ $1.1 \times 10^{-5} \mathrm{~m} \mathrm{~s}^{-1}$ ) is also 100 times higher than the value reported for $\mathrm{MB}$ degradation using a porous film, obtained by a spin-coated commercial $\mathrm{TiO}_{2}$ suspension $\left(k "=2.07 \times 10^{-7} \mathrm{~m} \mathrm{~s}^{-1}\right)$ [8]. A porous film evidently suffers from incomplete usage of the photocatalyst surface, due to either light penetration and/or mass diffusion limitation [8].

To determine the regime where the rate of reaction is dependent on the chemical reaction or the mass transfer, the microreactor is modeled for various values of 


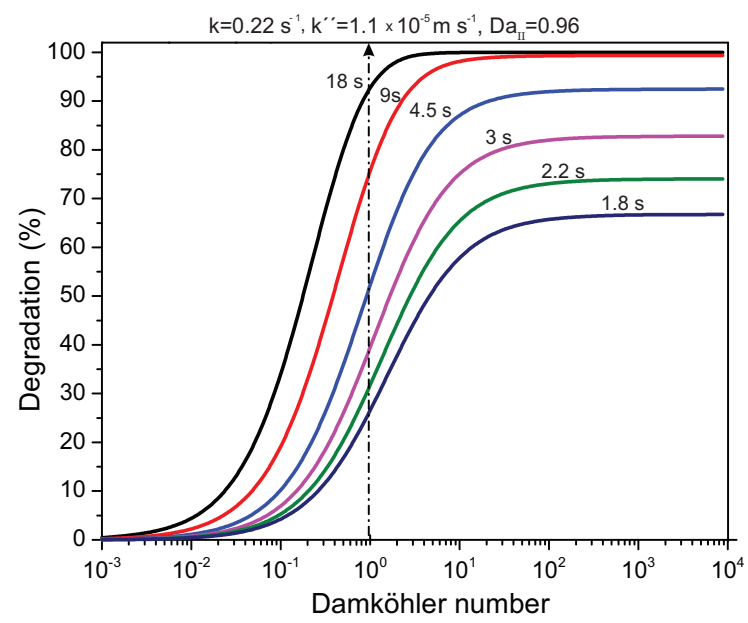

Figure 3.6: Degradation of $\mathrm{MB}$ as a function of second Damköhler number for the multiple residence times as computed by the model for the microreactor. The dashed line indicates the calculated second Damköhler based on the extracted reaction rate constant.

second Damköhler number which is defined as the ratio between the chemical reaction rate and the diffusive mass transport rate (equation3.11) [26].

$$
D a_{\mathrm{II}}=\frac{\text { reaction rate }}{\text { diffusive mass transfer rate }}=\frac{k^{\prime \prime} H}{D}
$$

Apparently, when $D a_{\mathrm{II}} \gg 1$ the chemical reaction rate is faster than transport of reactants to the catalyst and as a result the reaction rate becomes mass transport limited. Already for our microreactor, which displays efficient mass transport due to the small channel size, we obtain a $D a_{\text {II }}$ close to unity.

Based on the reported values in the literature, as presented in Table 3.1, typical Damköhler numbers much larger than unity can be realized. For such cases, the diffusive mass transport would be severely limiting. The mass transfer rate can be enhanced by convection (e.g. by stirring or flow) in order to reduce the diffusive boundary layer. Often the actual flow or stirring conditions are not mentioned in literature, which makes the identification of potential mass transfer limitations impossible.

Figure 3.6 shows the degradation of MB as a function of second Damköhler number ranging from $10^{-3}$ to $10^{4}$ and the dashed line indicates the calculated 
second Damköhler number based on our obtained $k "=1.1 \times 10^{-5} \mathrm{~m} \mathrm{~s}^{-1}$, microchannel height $(H)$ and the diffusion coefficient $(D)$ for MB. The microreactor operates in the transition of mass transfer limited and reaction limited region as the value for second Damköhler number is 0.96. This number can be used to assess the severance of mass transport limitations. Most easily, one can extract a height $H$ for $D a_{\mathrm{II}} \simeq 1$ that indicates a critical diffusive boundary layer thickness.

\subsubsection{Effect of photocatalyst thickness}

The effect of $\mathrm{TiO}_{2}$ film thickness on the photocatalytic performance was investigated. We prepared $\mathrm{TiO}_{2}$ thin films from $9 \mathrm{~nm}$ to $500 \mathrm{~nm}$ thickness and measured the degradation of $\mathrm{MB}$ at a single residence time of $1.78 \mathrm{~s}\left(50 \mu \mathrm{L} \mathrm{min}{ }^{-1}\right)$. As observed in Figure 3.7, catalysts thicker than $50 \mathrm{~nm}$ exhibit constant photocatalytic activity. In literature, different minimal thicknesses are reported ranging from 140 to $300-350 \mathrm{~nm}[6,29]$.

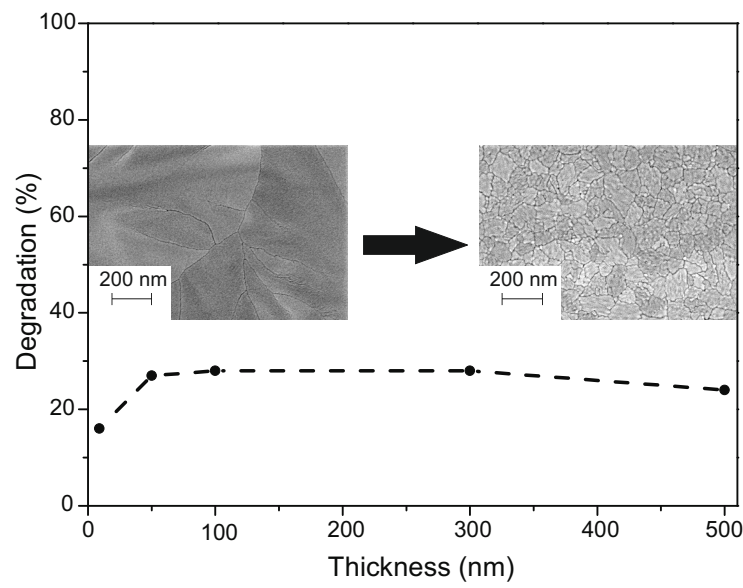

Figure 3.7: Photocatalytic degradation of $\mathrm{MB}$ as a function of catalyst thickness. The inserted HRSEM displays the surface morphology of a $9 \mathrm{~nm}$ (left) and a $50 \mathrm{~nm}$ (right) thin film.

In order to study the effect of film thickness intrinsically, it is of paramount importance that the surface morphology of the film does not change with film thickness. Luttrel et al. prepared thin anatase films utilizing high-quality epitaxial $\mathrm{TiO}_{2}$ films and evaluated the photocatalytic activity. They showed that the photocatalytic activity remained constant down to $\sim 5 \mathrm{~nm}$ due to the diffu- 
sion length of the charge carriers [4]. According to the HRSEM (Figure 3.7) the surface morphology of the thin films changes at $50 \mathrm{~nm}$ thickness. The density of the grains and as a result grain boundaries are significantly lower for the $9 \mathrm{~nm}$ thin film compared to the films thicker than $50 \mathrm{~nm}$. The extracted reaction rate constant for the $9 \mathrm{~nm}$ thin catalyst is $k^{\prime \prime}=7.3 \times 10^{-6} \mathrm{~m} \mathrm{~s}^{-1}$ which is only about a third lower compared to the thicker layers.

\subsection{Conclusion}

$\mathrm{TiO}_{2}$ was deposited by $\mathrm{DC}$ reactive magnetron sputtering at different oxygen partial pressures. A clear transition was observed as a function of the oxygen partial pressure between deposition of a metallic and an oxide film. The optical properties of the thin films were investigated by ellipsometry. After thermal annealing, anatase was formed, as identified by XRD. The performance of the photocatalyst was investigated in a microfluidic reactor by degradation of methylene blue for different residence times. $\sim 90 \%$ degradation was achieved within 20 $\mathrm{s}$ and a surface reaction rate constant of $1.1 \times 10^{-5} \mathrm{~m} \mathrm{~s}^{-1}$ was extracted. This is at least one order of magnitude higher than what reported in other studies. The photocatalytic activity of the sputtered $\mathrm{TiO}_{2}$ films is independent of thicknesses down to $50 \mathrm{~nm}$ at least. We present a numerical model that is capable to extract the intrinsic reaction rate constant. Furthermore, the model provides insight regarding possible mass transport limitations, verified by the second Damköhler number. This approach allows an intrinsic comparison of photocatalytic kinetic data. 


\section{Bibliography}

[1] A. L. Linsebigler, G. Lu, and J. T. Yates, Photocatalysis on $\mathrm{TiO}_{2}$ Surfaces: Principles, Mechanisms, and Selected Results, Chemical Reviews 95, 735 (1995).

[2] A. Fujishima and K. Honda, Electrochemical Photolysis of Water at a Semiconductor Electrode, Nature 238, 37 (1972).

[3] S. Banerjee and J. Gopal, Physics and chemistry of photocatalytic titanium dioxide: visualization of bactericidal activity using atomic force microscopy, Current Science 90, 1378 (2006).

[4] T. Luttrell, S. Halpegamage, J. Tao, A. Kramer, E. Sutter, and M. Batzill, Why is anatase a better photocatalyst than rutile?-Model studies on epitaxial $\mathrm{TiO}_{2}$ films., Sci. Rep. 4, 4043 (2014).

[5] D. a. H. Hanaor and C. C. Sorrell, Review of the anatase to rutile phase transformation, Journal of Materials Science 46, 855 (2010).

[6] K. Eufinger, D. Poelman, H. Poelman, R. D. Gryse, and G. B. Marin, Effect of Microstructure and Crystallinity on the Photocatalytic Activity of $\mathrm{TiO}_{2}$ Thin films Deposited by dc Magnetron Sputtering, Journal of Physics D: Applied Physics 40, 5232 (2007).

[7] L.-D. Piveteau, B. Gasser, and L. Schlapbach, Evaluating mechanical adhesion of solgel titanium dioxide coatings containing calcium phosphate for metal implant application, Biomaterials 21, 2193 (2000).

[8] A. Visan, D. Rafieian, W. Ogieglo, and R. G. H. Lammertink, Modeling intrinsic kinetics in immobilized photocatalytic microreactors, Applied Catalysis, B: Environmental 150-151, 93 (2014).

[9] T.-S. Yang, C.-B. Shiu, and M.-S. Wong, Structure and Hydrophilicity of Titanium Oxide Films prepared by Electron Beam Evaporation, Surface Science 548, 75 (2004). 
[10] A. Manivannan, N. Spataru, K. Arihara, and A. Fujishima, Electrochemical Deposition of Titanium Oxide on Boron-Doped Diamond Electrodes, Electrochemical and Solid-State Letters 8, C138 (2005).

[11] L. M. Williams, Structural properties of titanium dioxide films deposited in an rf glow discharge, Journal of Vacuum Science \& Technology, A: Vacuum, Surfaces, and Films 1, 1810 (1983).

[12] T. Kubart, J. Jensen, T. Nyberg, L. Liljeholm, D. Depla, and S. Berg, Influence of the target composition on reactively sputtered titanium oxide films, Vacuum 83, 1295 (2009).

[13] Y. Suda, H. Kawasaki, T. Ueda, and T. Ohshima, Preparation of high quality nitrogen doped $\mathrm{TiO}_{2}$ thin film as a photocatalyst using a pulsed laser deposition method, Thin Solid Films 453-454, 162 (2004).

[14] S. A. O’Neill, I. P. Parkin, R. J. H. Clark, A. Mills, and N. Elliott, Atmospheric pressure chemical vapour deposition of titanium dioxide coatings on glass, Journal of Materials Chemistry 13, 56 (2003).

[15] J. Aarik, A. Aidla, H. Mändar, and T. Uustare, Atomic Layer Deposition of Titanium Dioxide from $\mathrm{TiCl}_{4}$ and $\mathrm{H}_{2} \mathrm{O}$ : Investigation of Growth Mechanism, Applied Surface Science 172, 148 (2001).

[16] M. Yamagishi, S. Kuriki, P. Song, and Y. Shigesato, Thin film TiO 2 Photocatalyst Deposited by Reactive Magnetron Sputtering, Thin Solid Films 442, 227 (2003).

[17] D. Dumitriu, A. R. Bally, C. Ballif, P. Hones, P. Schmid, R. Sanjinés, F. Lévy, and V. Pârvulescu, Photocatalytic degradation of phenol by $\mathrm{TiO}_{2}$ thin films prepared by sputtering, Applied Catalysis, B: Environmental 25, 83 (2000).

[18] S. Takeda, S. Suzuki, H. Odaka, and H. Hosono, Photocatalytic TiO ${ }_{2}$ Thin film Deposited onto Glass by DC Magnetron Sputtering, Thin Solid Films 392, 338 (2001).

[19] S.-M. Chiu, Z.-S. Chen, K.-Y. Yang, Y.-L. Hsu, and D. Gan, Photocatalytic Activity of Doped $\mathrm{TiO}_{2}$ Coatings prepared by Sputtering Deposition, J. Mater. Process. Technol. 192-193, 60 (2007).

[20] Y. Pihosh, M. Goto, A. Kasahara, and M. Tosa, Photocatalytic Property of $\mathrm{TiO}_{2}$ Thin films Sputtered-Deposited on Unheated Substrates, Applied 
Surface Science 256, 937 (2009).

[21] J. Yu and X. Zhao, Effect of surface treatment on the photocatalytic activity and hydrophilic property of the sol-gel derived $\mathrm{TiO}_{2}$ thin films, Materials Research Bulletin 36, 97 (2001).

[22] P. Sangpour, Photoenhanced Degradation of Methylene blue on Cosputtered M: $\mathrm{TiO}_{2}(\mathrm{M}=\mathrm{Au}, \mathrm{Ag}, \mathrm{Cu})$ Nanocomposite Systems: a Comparative Study, J. Phys. Chem. C 2, 13955 (2010).

[23] M. Ratova, P. Kelly, G. West, and I. Iordanova, Enhanced Properties of Magnetron Sputtered Photocatalytic Coatings via Transition Metal Doping, Surface and Coatings Technology 228, S544 (2013).

[24] P. B. Nair, G. P. Daniel, K. Joy, V. Ramakrishnan, D. D. Kumar, and P. Thomas, Structural, Optical, Photoluminescence and Photocatalytic Investigations on Fe Doped $\mathrm{TiO}_{2}$ Thin films, Thin Solid Films 550, 121 (2014).

[25] B. Johs and J. S. Hale, Dielectric Function representation by B-splines, Physica Status Solidi A: Applications and Materials Science 205, 715 (2008).

[26] R. B. Bird, W. E. Stewart, and E. N. Lightfoot, Transport phenomena, 2nd ed. (John Wiley \& Sons, Inc., 2007), pp. 543-581.

[27] C. J. Tavares, J. Vieira, L. Rebouta, G. Hungerford, P. Coutinho, V. Teixeira, J. O. Carneiro, and A. J. Fernandes, Reactive sputtering deposition of photocatalytic $\mathrm{TiO}_{2}$ thin films on glass substrates, J. Mater. Sci. Eng. B 138, 139 (2007).

[28] M. Horprathum, A Spectroscopic Ellipsometry Study of $\mathrm{TiO}_{2}$ Thin films Prepared by dc Reactive Magnetron Sputtering: Annealing Temperature Effect, Chin. Phys. Lett. 24, 1505 (2007).

[29] H. Tada and M. Tanaka, Dependence of $\mathrm{TiO}_{2}$ Photocatalytic Activity upon its Film Thickness, Langmuir 13, 360 (1997). 



\section{CHAPTER 4}

\section{In-situ AFM study of $\mathrm{Si} / \mathrm{TiO}_{2}$ heterojunctions}

Patterned $\mathrm{TiO}_{2}$ thin films were prepared on p-Si, n-Si and p-Si/6 $\mu \mathrm{m}$ $\mathrm{SiO}_{2}$ substrates. The interaction force between silica tip and $\mathrm{TiO}_{2}$ was monitored using a state-of-the-art high resolution AFM under UV illumination. The corresponding surface charges were extracted using a model based on DLVO theory with a charge regulating boundary. It was demonstrated that the interaction forces and corresponding surface charges are significantly influenced by the type of substrates. The photocatalytic activity of the $\mathrm{TiO}_{2}$ thin films on different substrates were assessed in a microreactor. High degradation of methylene blue (MB) (90\% degradation within $20 \mathrm{~s}$ residence time) was observed for $\mathrm{p}-\mathrm{Si} / \mathrm{TiO}_{2}$ due to the enhanced charge separation which was in good agreement with the AFM observations. 


\subsection{Introduction}

In recent years there has been an increasing interest in utilizing photocatalysis for environmental remediation. $\mathrm{TiO}_{2}$ among other semiconductors has been identified as the most widely employed photocatalyst due to the characteristics like non-toxicity, abundance, chemical inertness and being relatively cheap. Photocatalytic reactions using $\mathrm{TiO}_{2}$ involves illumination of titanium dioxide with light possessing an energy equal or higher than the band gap of titanium dioxide. This leads to excitation of electrons to the conduction band leaving behind a hole in the valence band of the $\mathrm{TiO}_{2}$. These generated electron-hole pairs in turn will participate in generation of active species such as hydroxyl radicals and superoxide ions which in turn trigger the degradation process of the organic compounds. Quick recombination of the generated electron-hole pairs has been identified as a hindering effect on the photocatalytic processes. Employing noble metals as co-catalyst $[1,2]$ and forming a Schottky type photochemical diode [3] is one of the most commonly employed methods to prevent the recombination of the photogenerated electron-hole pairs and thus improve photocatalytic processes.

There have been few studies on using semiconductors as co-catalysts to reduce recombination $[3,4]$. By selecting semiconductors with different energy band structures, the excited electrons and holes are transfered between them thus contributing to charge carriers separation. Coupled heterostructures (two semiconductor types) are categorized into two groups based on their doping level; n-type semiconductors combined with n-type semiconductors forming n-n junctions or n-type semiconductors combined with p-type semiconductors forming $\mathrm{p}$ - $\mathrm{n}$ junctions. A common example for the former group is $\mathrm{p}-25 \mathrm{TiO}_{2}$, i.e one of the most active commercial photocatalyst, which is a mixture of rutile and anatase polymorphs of $\mathrm{TiO}_{2}$ forming an n-n junction. The high photocatalytic performance is attributed to the presence of electron trapping sites and enhanced interfacial charge transfer between rutile and anatase. This leads to better charge separation and lower recombination of charge carriers accordingly [5]. Other examples include $\mathrm{WO}_{3} / \mathrm{TiO}_{2}$ [6], $\mathrm{PbO}_{2} / \mathrm{TiO}_{2}$ [7] and $\mathrm{BiO}_{\mathrm{x}} / \mathrm{TiO}_{2}$ [8]. The second group concern $\mathrm{p}-\mathrm{n}$ junctions which receive considerable attention due to their powerful charge separation potential. In principle they function as a conven- 
tional diode whereby the electrical field causes the separation of electron and hole. A few studies have reported the use p-n junctions in photocatalysis including $\mathrm{Cu}_{2} \mathrm{O} / \mathrm{TiO}_{2}[9]$ and $\mathrm{NiO} / \mathrm{TiO}_{2}[10]$ where higher photocatalytic activities were observed compared to $\mathrm{TiO}_{2}$.

In all these studies the performance of the photocatalyst is evaluated based on the degradation of specific compounds. This causes inconsistency in evaluation of the photocatalyst considering different reactors and model compounds. The photoactivity of all photocatalyst originates from their electronic structure such as their energy band structure. Thus in order to have a reliable benchmark, in-situ measurements of the surface morphology, charges and electrochemical phenomena at the solid-liquid interface is of paramount importance.

In recent years, vacuum and ambient environment Scanning tunneling microscopy (STM) and Kelvin probe force microscopy (KPFM) studies have been spectacularly successful and provided detailed insight into the surface structure and defects of $\mathrm{TiO}_{2}$ surfaces, as well as revealing catalytic processes by mapping the electric surface potential of surfaces. The surface force is often measured using a surface force apparatus (SFA) or a total internal reflectance microscope [11]. New scanning force microscopes (SFM), i.e., conducting-probe SFM, scanning capacitance microscopy, electro-static force microscopy, and Kelvin probe force microscopy, hold great promise for electronic characterization of catalytic materials since they allow simultaneous high-resolution topographic imaging as well as electrical characterization. While in situ atomic resolution imaging, revealing catalytic processes in vacuum, has nowadays become routine, extensions of these capabilities to liquid environments are scarce. For instance Siretanu et al. [12] directly investigate the structure of ions adsorbed on the surface of gibbsite $(\alpha-$ $\left.\mathrm{Al}(\mathrm{OH})_{3}\right)$ using atomic force microscopy (AFM).

Here, we utilize state-of-the-art high resolution AFM to image in situ, the forces between a silica probe and anatase $\mathrm{TiO}_{2}$ in an aqueous electrolyte solution. The effect of different substrates (p-Si, n-Si and p-Si/6 $\mu \mathrm{m} \mathrm{SiO}_{2}$ ) on the surface properties of the $\mathrm{TiO}_{2}$ covering film was investigated. We probed in situ the local surface charge density at the $\mathrm{TiO}_{2}$-electrolyte interface. We demonstrate that the surface charge density varies upon illumination, depending on the nature of the substrate supporting the thin $\mathrm{TiO}_{2}$ films. The corresponding pho- 
tocatalytic activities were evaluated using a microreactor and intrinsic surface averaged reaction rate constants were extracted using a model which includes convection and diffusion inside the microchannel.

\subsection{Experimental}

\subsubsection{Patterned $\mathrm{TiO}_{2}$ thin film preparation}

Silicon wafers p-type (100), n-type (100) and p-type with $6 \mu \mathrm{m}$ thermally grown oxide are used as the substrates. They were cleaned in $98 \%$ nitric acid for 10 minutes followed by rinsing and drying prior to deposition. 2-5 nm native oxide (from here on referred as silica) was present on ( $p$ and $n$ )-Si substrates. The patterned structure of $\mathrm{TiO}_{2}$ thin films was prepared by photolithography using image reversal photoresist. The complete process steps are schematically illustrated in figure 4.1. To increase the adhesion of the photoresist, hexamethyldisilazane (HMDS) was spincoated for 30 seconds at $4000 \mathrm{rpm}$. This was followed by spincoating of an image reversal photoresist (TI35ES, Microchemicals) for 30 seconds at $4000 \mathrm{rpm}$ (Figure 4.1 (a)). After prebaking at $95{ }^{\circ} \mathrm{C}$ for 120 seconds the substrate was exposed for 23 seconds (Figure 4.1 (b)). At this step the exposed area becomes soluble. This was followed by a reversal bake at $120{ }^{\circ} \mathrm{C}$ for 120 seconds and a flood exposure (without photomaks) for 60 seconds leading to enhanced solubility of the exposed area in the previous step and crosslinking of the unexposed areas. Finally the photoresist was developed for 120 seconds in developer and spin dried (Figure 4.1 (c) and corresponding SEM micrograph (c)). Subsequently, $\mathrm{TiO}_{2}$ thin films were deposited using a custom made DC magnetron sputtering system (TCOATER, MESA+ Nanolab) in cleanroom environment (Figure 4.1 (d)). A $10 \mathrm{~cm}$ diameter titanium disk of $99.7 \%$ purity was used as the deposition target. The substrate-target distance was set at 4.4 $\mathrm{cm}$ and the substrate rotated at $5 \mathrm{rpm}$ during the whole deposition process for enhanced uniformity. The sputtering chamber was evacuated to a base pressure of $10^{-7}$ mbar at room temperature prior to the deposition. A mixture of $99.5 \%$ pure oxygen and $99.99 \%$ pure argon were used as the sputtering and reactive gas respectively. The total process pressure was regulated at $6 \times 10^{-3}$ mbar in all 
the experiments. The target was powered by a variable DC-power supply at 500 $\mathrm{W}$ and pre-sputtered for 2 minutes with a shutter covering the substrates in all experimental trials. The argon and oxygen flow rate was set to 30 and $6 \mathrm{sccm}$ respectively for the deposition of stoichiometric $\mathrm{TiO}_{2}$ [13]. Following the deposition the photoresist was removed (lift-off) by ultrasonic immersion in acetone for 10 minutes. The resulting patterned $\mathrm{TiO}_{2}$ thin film on the silicon substrate is shown in figure 4.1 (e) and the corresponding SEM micrograph (e). The lift-off process $\mathrm{TiO}_{2}$ thin films were annealed in an atmospheric environment for $8 \mathrm{~h}$ at $500{ }^{\circ} \mathrm{C}$ with heating and cooling rates of $2{ }^{\circ} \mathrm{C} \mathrm{min}-1$. The crystal structure of the thin films on different substrates was investigated by XRD (Bruker D2) using CuK- $\alpha$ radiation at $40 \mathrm{kV}$ and $40 \mathrm{~mA}$ working in the $\theta-2 \theta$ mode. The morphology and structure of $\mathrm{TiO}_{2}$ samples are also analysed by scanning electron microscopy (SEM).

\subsubsection{Atomic force microscopy}

The detailed description is given elsewhere [12]. Briefly AFM imaging was performed using a Multimode 8 AFM with Nanoscope V controller (Bruker Nano). The AFM images shown in this chapter were obtained using FASTSCAN-B cantilevers $\left(f_{0}=170 \mathrm{kHz}, c_{z}=3 \mathrm{~N} / \mathrm{m}, \mathrm{Q}=10\right.$, tip radius $\left.\sim 3 \mathrm{~nm}\right)$, cleaned prior to imaging by rinsing with a mixture of ethanol and isopropanol $(\sim 1: 1)$ and additional ambient air plasma treatment (Harrick Plasma) for $15 \mathrm{~min}$. The cantilevers were mounted in a fluid cell and fully immersed into the liquid for the experiment. To minimize the drift, the system was allowed to thermally equilibrate at room temperature for 20-60 min before acquiring any data. All images were collected in liquid with the AFM on a vibration isolation stand. Taping mode or amplitude modulation mode (AM-AFM) was used throughout all the experiments with free amplitudes $A_{0}$ typically smaller than $1 \mathrm{~nm}$. The force was kept at the lowest possible value to minimize the forces of interaction between the tip and the surface. The ratio of the set point amplitude was maintained to the free amplitude $\left(\mathrm{A} / A_{0}\right)$ at 0.9 . The linear scanning rate was optimized between 1 and $15 \mathrm{~Hz}$ with scan resolution of 512 samples per line. The surface charge from the AFM measurement at $\mathrm{TiO}_{2}$-electrolyte interface was extracted using the method presented in [14]. 
a)

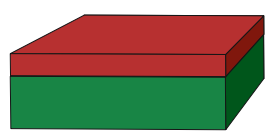

c)

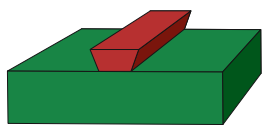

b)

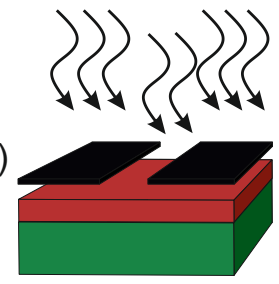

d)

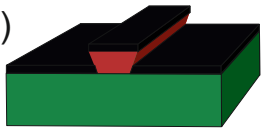

e)
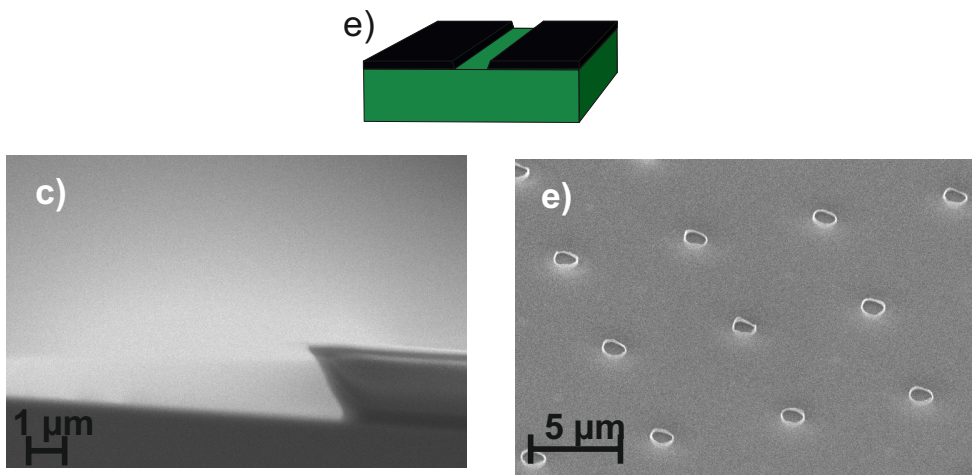

Figure 4.1: Schematic illustration of the patterned $\mathrm{TiO}_{2}$ thin film preparation steps; a) Spin coating of the image reversal photoresist on silicon. b) Exposure of the photoresist under a photomask c) Development of the resist and formation of the undercut (SEM (c)). d) Magnetron sputtering of $\mathrm{TiO}_{2}$ thinfilm. e) Photoresist lift-off and the formation of the patterned $\mathrm{TiO}_{2}$ thinfilm (SEM (e)).

\subsubsection{Photocatalytic measurement}

Photocatalytic performance of $\mathrm{TiO}_{2}$ deposited on n-type, p-type silicon and $6 \mu \mathrm{m}$ $\mathrm{SiO}_{2}$ on p-type silicon substrates were assessed in a microreactor. A microstructured mold was made by standard photolithography using negative photoresist (SU-8) in cleanroom environment. SU-8 was spun on silicon wafer at $500 \mathrm{rpm}$ for 10 seconds and continued to $100 \mathrm{rpm}$ for 30 seconds. Then the sample was pre-baked at 50,65 , and $95{ }^{\circ} \mathrm{C}$ for 10,10 , and 45 minutes, respectively. This was followed by UV exposure for 33 seconds and post baking at 50,65 , and $80{ }^{\circ} \mathrm{C}$ for 5, 10, and 20 minutes, respectively. Finally the sample was spray developed by RER 600 . The SU-8 height was measured by an optical profiler (Bruker WLI 
Contour GT-I). Polydimethylsiloxane (PDMS) was prepared by mixing the polymer base (Permacol RTV-615 A) with the curing agent (Permacol RTV-615 B) in a 10:1 mass ratio, followed by degassing in a dessicator. The PDMS was cast on the mold and further degassed in a dessicator and cured at $60{ }^{\circ} \mathrm{C}$ for 45 minutes. The PDMS slab was removed from the mold and inlets and outlets were punctured. Subsequently, it was attached to the $\mathrm{TiO}_{2}$ substrate. To ensure a leakfree assembly, additional PDMS was slightly pre-cured and poured at the edge of the previously cured PDMS cap and further cured overnight. The fabrication steps are schematically illustrated in figure 4.2. The channel has a rectangular cross section with $50 \mu \mathrm{m}$ height, $500 \mu \mathrm{m}$ width and $5.96 \mathrm{~cm}$ length, giving a total volume of $1.49 \mu \mathrm{L}$. An aqueous solution of $40 \mu \mathrm{M}$ methylene blue (MB) was injected into the microreactor with flow rates between 3 and $50 \mu \mathrm{L} \mathrm{min}^{-1}$ using a syringe pump (New Era NE-4000). The MB concentration at the outlet was determined by light absorbance measurements using an in-line UV-vis spectrometer (USB2000+ Miniature Fiber Optic Ocean Optics). The degradation experiments were carried out using a UV light source (HP-120 Opystec Dr. Gröbel) fixed at a distance of $4 \mathrm{~cm}$ from the catalyst surface providing $100 \mathrm{~mW} / \mathrm{cm}^{2}$ photon flux density at $365 \mathrm{~nm}$ wavelength. The surface reaction rate constant was extracted using a model which includes convection and diffusion in the microchannel [15].

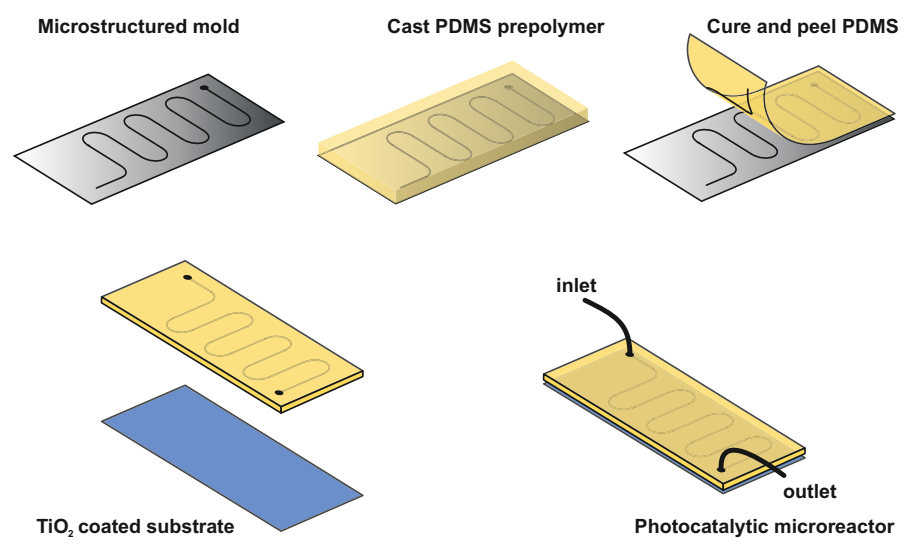

Figure 4.2: Illustration of the microreactor fabrication. The flow channel is defined in PDMS which is subsequently attached to the $\mathrm{TiO}_{2}$ surface on different substrates. 


\subsection{Results and Discussion}

\subsubsection{AFM}

All AFM experiments were performed in slightly acidic (pH 6) and $10 \mathrm{mM} \mathrm{NaCl}$ aqueous electrolyte using silicon AFM tips. Figure 4.3 (a) shows the AFM image of the patterned $\mathrm{TiO}_{2}$ thin film (top) and corresponding lateral topographic profile (middle) and SEM micrographs (bottom). The diameter of the holes is $1 \mu \mathrm{m}$ (figure 4.3 (b)). The film has a dense structure and a reasonably smooth surface morphology and high magnification image displays crystal grains (figure 4.3 (c)).
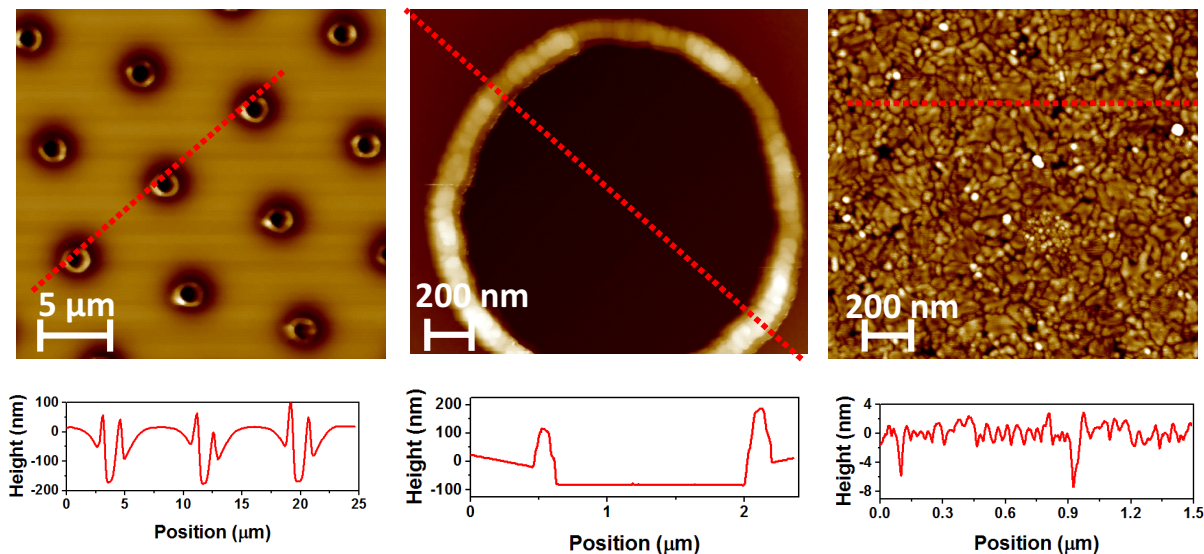

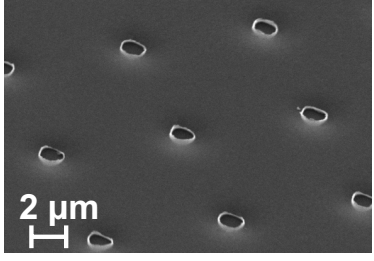

a)

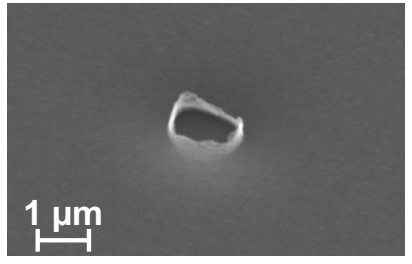

b)

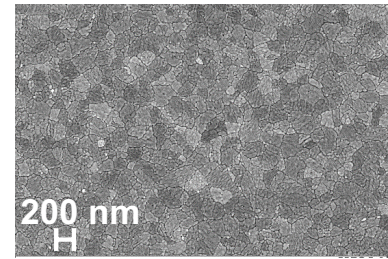

c)

Figure 4.3: Topographic AFM image, lateral topographic profile and SEM micrographs of the patterned $\mathrm{TiO}_{2}(\mathrm{a})$, high magnification of the hole (b) and surface of the $\mathrm{TiO}_{2}$ (c).

Figure 4.4 depicts an example of a 2-dimensional (2D) force field generated by measuring 300 tip-sample interaction curves over the $\mathrm{TiO}_{2} / \mathrm{SiO}_{2}$ surface along a line of $4.5 \mu \mathrm{m}$ length in $\mathrm{x}$-direction. The two dimensional interaction force 
map reveals that on the silica region forces gradually increases from zero (green) to repulsive value (orange-red) over a distance of several nanometers. In the case of $\mathrm{TiO}_{2}$ the tip is attracted towards the surface (attractive force) (blue) indicating an opposite, i.e. positive, surface charge on the $\mathrm{TiO}_{2}$ surface. This is expected based on the isoelectric points of anatase $\mathrm{TiO}_{2}(\mathrm{pK} \sim 6.2$ [16]) and silica (pK 2 [11]) which are immersed in slightly acidic (pH 5.8-6) $10 \mathrm{mM} \mathrm{NaCl}$ solution. Compared to the silica regions, there is more lateral variation in the force, indicating a larger degree of heterogeneity on the $\mathrm{TiO}_{2}$ surface.

Moreover, as described in the Zhao paper [14], a method has been developed based on the analysis of tip-sample force-distance curves in the framework of Derjaguin-Landau-Verwey-Overbeek (DLVO) theory using a charge regulation (CR) boundary condition for the Poisson-Boltzmann equation in combination with a surface complexation model to describe the surface charge/potential of surfaces. The resulting surface charge profile after analysis of 300 tip-sample interaction forces individually, has been plotted as a black line (figure 4.4(b)). From the charge profile, it is clear that silica is negatively charged, while titania is positively charged presenting a heterogeneous charge distribution. The positive charge is in agreement with the macroscopic observation of Holmberg et al. [17] where a positive surface charge for $\mathrm{TiO}_{2}$ was measured by $\zeta$-potential measurements.

Figure 4.5 shows the surface charge (a) and 2-dimensional (2D) force field generated by measuring 300 tip-sample interaction curves over the $\mathrm{TiO}_{2} / \mathrm{SiO}_{2}$ (silica) surface along a line of $4.5 \mathrm{~m}$ length in x-direction under UV illumination. No differences in terms of surface charge and force was observed for the silica region. On $\mathrm{TiO}_{2}$, the effect of light was significant, as the existing attractive force between silica tip and $\mathrm{TiO}_{2}$ became less attractive (figure 4.5(b)) with surface charge becoming less positive (figure 4.5(b)) under UV illumination.

Figure 4.6 presents the band structure of $\mathrm{n}-\mathrm{Si} / \mathrm{TiO}_{2}, \mathrm{p}-\mathrm{Si} / \mathrm{TiO}_{2}$ and $\mathrm{p}-\mathrm{Si} / 6 \mu \mathrm{m}$ $\mathrm{SiO}_{2} / \mathrm{TiO}_{2}$ and the corresponding tip-surface interactions under UV illumination. At the interface of the $\mathrm{TiO}_{2}$ and electrolyte the bands bend downward in all the samples. Generally when a n-type semiconductor is in contact with a electrolyte the electrons are transfered from the semiconductor to the electrolyte due to the fact that the Fermi level of the semiconductor is higher than the redox potential 

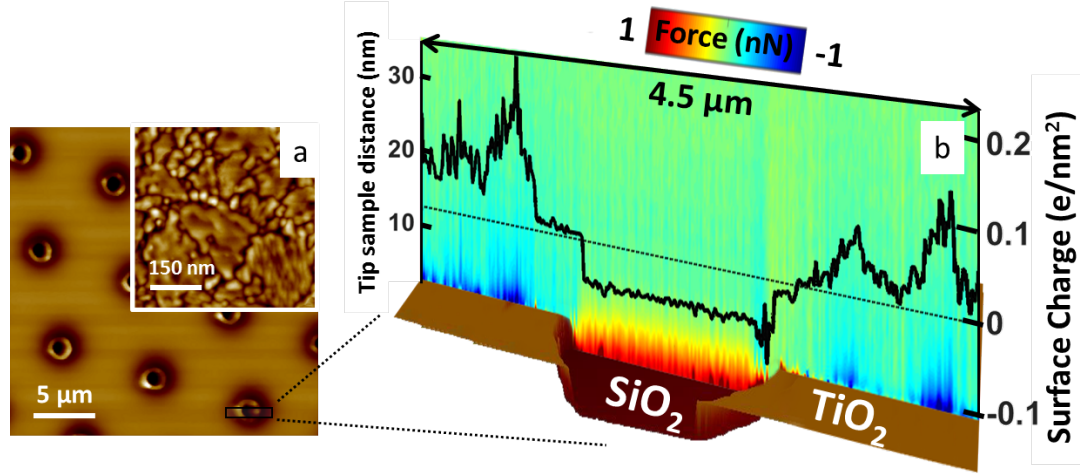

Figure 4.4: a) Topography image of patterned $\mathrm{TiO}_{2}$ and high magnification of the surface (insert), (b) color-coded 2D force field generated from 300 tip-sample interaction curves in $10 \mathrm{mM} \mathrm{NaCl}$ at $\mathrm{pH} \sim 6$ over the $\mathrm{TiO}_{2} / \mathrm{SiO}_{2}$ surface along a line of $4.5 \mathrm{~m}$ length in x-direction (blue: attractive force; red: repulsive force; green: zero force; see scale bar). Solid black line superimposing the $2 \mathrm{D}$ force field represents the surface charge values.

of the electrolyte $[18,19]$. This leads to the formation of a depletion layer and positive charge in the semiconductor. Hence the electron transfer is hampered by the negative charges in the electrolyte and the band bends upward.

The position of the band edges is also dependent on the surface charge of the semiconductor. Since the experiment was performed at $\mathrm{pH} \sim 5.8$ which is lower than the $\mathrm{TiO}_{2}$ isoelectric point, the surface of $\mathrm{TiO}_{2}$ is positively charged. Thus the band bends downwards (figure 4.6). As observed in figure 4.6, the effect of the substrate on the surface charge under illumination is pronounced. On ptype/ $\mathrm{TiO}_{2}$ junctions the generated electron-holes are efficiently separated while in the n-type substrate the elecron-hole pairs recombine. When p-Si and n- $\mathrm{TiO}_{2}$ was illuminated the excited electrons will move from the $\mathrm{CB}$ of $\mathrm{p}-\mathrm{Si}$ to the $\mathrm{CB}$ of $\mathrm{TiO}_{2}$. Hence the attractive force between positively charged $\mathrm{TiO}_{2}$ and AFM tip becomes less. In the case of the n-type silicon and $n-\mathrm{TiO}_{2}$ heterojunction (n- $\mathrm{n}$ ) the generated electrons in $\mathrm{TiO}_{2}$ are transfered to n-Si. The holes, due to the positive surface charge, follow the same path. Therefore, generated electron-hole pairs recombine and the surface charge on $\mathrm{TiO}_{2}$ remains unchanged. Thus on the $\mathrm{n}-\mathrm{Si} / \mathrm{TiO}_{2}$ the illumination has no effect on the surface charge. The presence of a thick $\mathrm{SiO}_{2}$ on the p-type silicon hinders the separation of electron and hole 


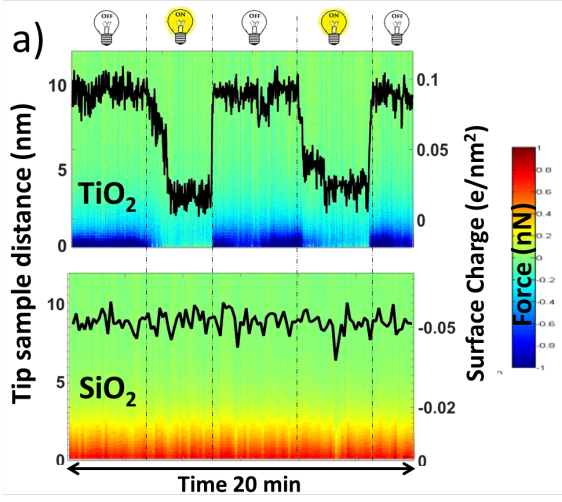

b)

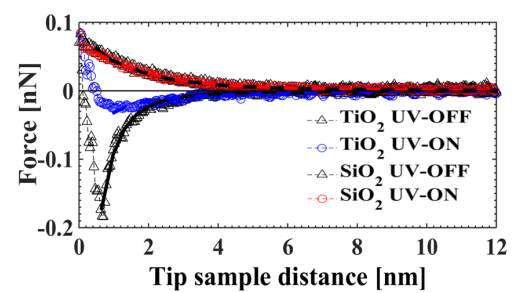

Figure 4.5: (a) Surface charge values (black solid lines) superimposed on color coded 2D force field generated from 1050 tip-sample interaction curves recorded vs. time on $\mathrm{TiO}_{2}$ (top) and $\mathrm{SiO}_{2}$ (bottom) in $10 \mathrm{mM} \mathrm{NaCl}$ solution under alternating UV light onoff irradiation. (blue: attractive force; red: repulsive force; green: zero force; see scale bar). b) Tip sample interaction forces on the silica and $\mathrm{TiO}_{2}$ in $10 \mathrm{mM} \mathrm{NaCl}$ in dark and under illumination. Dotted lines are the experimental curves and the solid lines are the theoretically fitted force curves with DLVO theory.

and as a consequence no effect of light on the surface charge was observed. It should be noted that electron-hole pairs are also generated inside the silicon (n and p type) considering its small band gap (1.12 eV).

\subsubsection{Photocatalytic assessment}

Prior to any photocatalytic assessment the presence of anatase phase was confirmed by XRD for deposited $\mathrm{TiO}_{2}$ on all the substrates (figure 4.7). Figure 4.8 shows the degradation of $\mathrm{MB}(40 \mu \mathrm{M})$ in the microreactor as a function of residence times utilizing the $\mathrm{TiO}_{2}$ on p-type silicon. The extracted surface reaction rate constant for our sputtered film $\left(k "=1.1 \times 10^{-5} \mathrm{~m} \mathrm{~s}^{-1}\right)$ is at least an order of magnitude larger compared to previously reported values [15]. The extracted surface reaction rate constant is also 100 times higher than the value reported for MB degradation using a porous $\mathrm{TiO}_{2}$ film and p-Si [20]. The higher surface reaction rate constant may be explained by the fact that the dense morphology and structure of the $\mathrm{TiO}_{2}$ film prepared by sputtering is beneficial for the electronic properties of the stack. In addition, the superior charge separation in the p-n junction may be responsible for the high surface reaction rate constant. It 


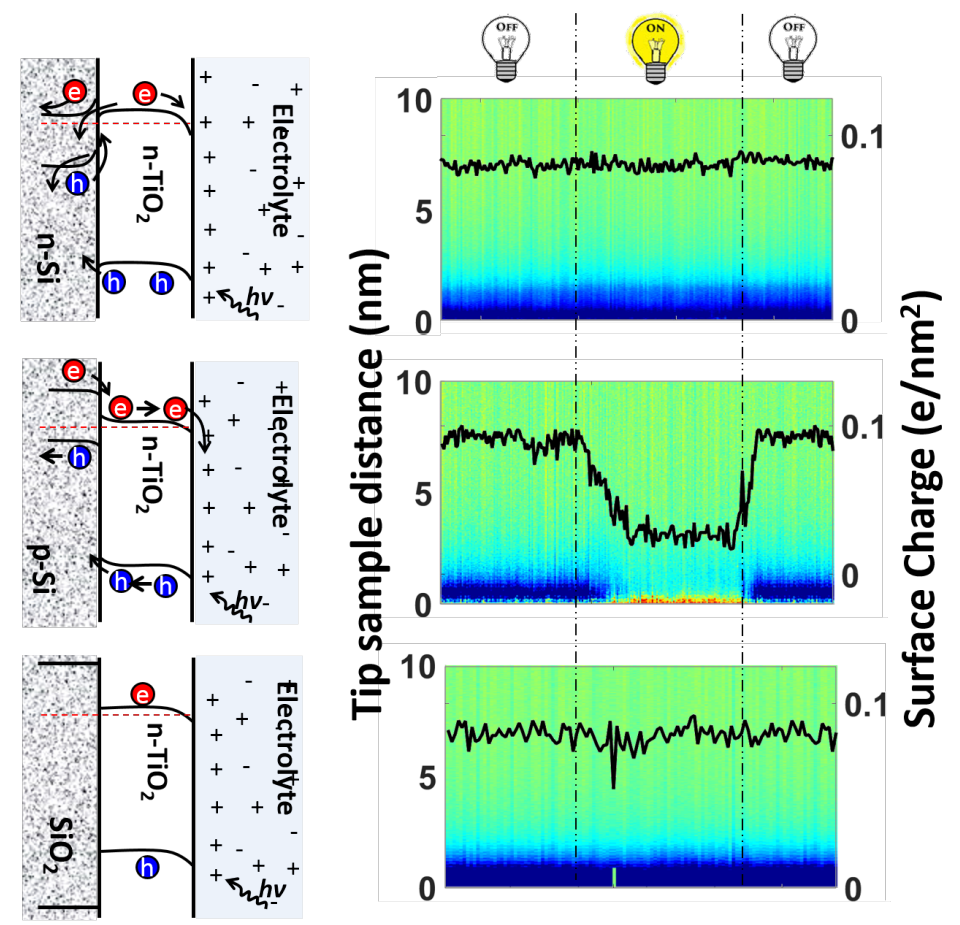

Figure 4.6: Electronic band structure (left side) and (right side) surface charge values (black solid lines) superimposed on color coded 2D force field generated from 300 tipsample interaction curves recorded vs. time on $200 \mathrm{~nm} \mathrm{TiO}_{2}$ film on n-type (100) silicon wafer (top), $200 \mathrm{~nm} \mathrm{TiO}$ film on p-type (100) silicon wafer (middle) and $200 \mathrm{~nm} \mathrm{TiO}_{2}$ film on $6 \mathrm{~m}$ thermally grown oxide (bottom) in $10 \mathrm{mM} \mathrm{NaCl}$ solution under alternating UV light on-off irradiation. (blue: attractive force; red: repulsive force; green: zero force; see scale bar).

has been widely reported that the photocatalytic activity of $\mathrm{TiO}_{2}$ is closely dependent on the effective electron-hole pair separation. As observed in figure 4.8 (insert) the conduction band of anatase $\mathrm{TiO}_{2}(-5.1 \mathrm{eV}$ [21]) is lower than the conduction band of p-Si $(-4.97[22])$, thus $\mathrm{TiO}_{2}$ becomes a sink for generated electrons under illumination.

The accumulation of the electrons were confirmed by in-situ AFM shown in figure 4.6 (middle). The excited electrons in the p-Si conduction band are transfered to the conduction band of $\mathrm{TiO}_{2}$ and holes from the $\mathrm{TiO}_{2}$ valence band are moved to the valence band of $\mathrm{p}-\mathrm{Si}$. Due to the reduction of adsorbed oxygen on the surface with the accumulated electrons powerful oxidizing agents $\left(\mathrm{O}_{2}{ }^{\cdot-}\right)$ are 


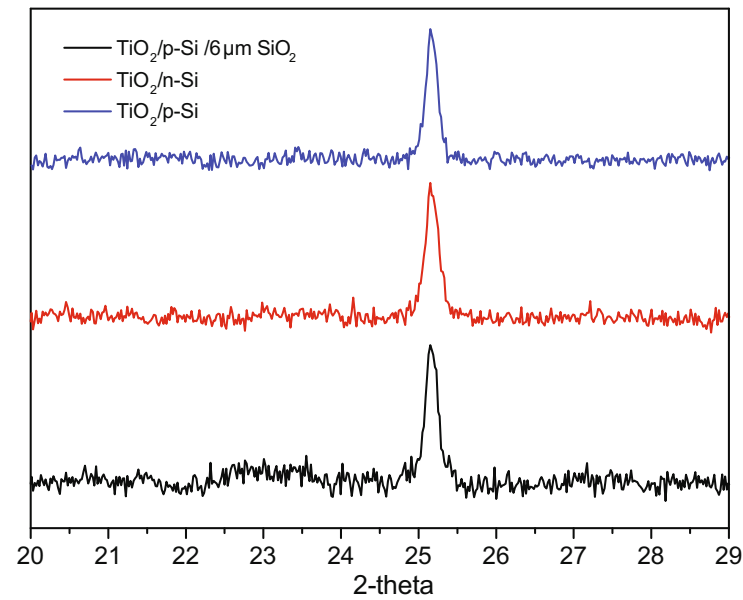

Figure 4.7: XRD analysis of $\mathrm{TiO}_{2}$ on different substrates. The anatase phase is present in all the samples.

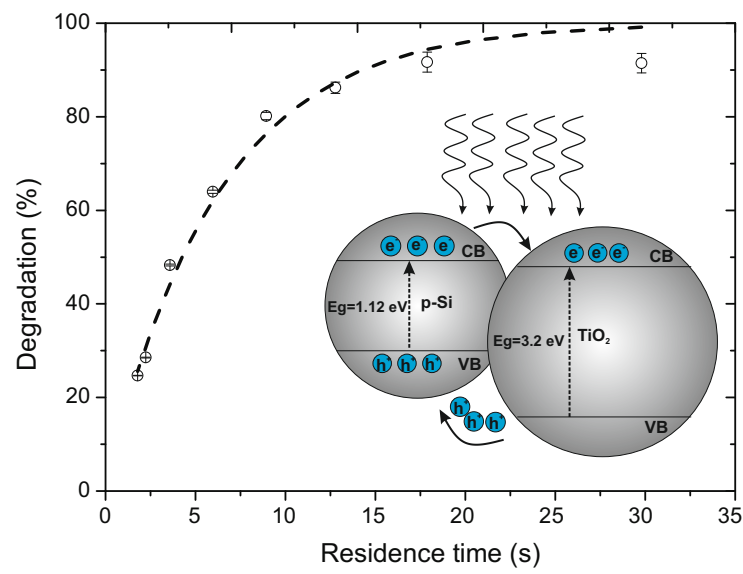

Figure 4.8: Degradation of methylene blue $(40 \mu \mathrm{M})$ as a function of residence time. The fit results from the numerical model using a single fitted surface reaction rate constant $\left(k "=1.1 \times 10^{-5} \mathrm{~m} \mathrm{~s}^{-1}\right)$. The insert shows the mechanism of charge separation in $\mathrm{p}-\mathrm{Si} / \mathrm{TiO}_{2}$.

formed which degrade the dye molecules [23].

Different types of semiconductor heterojunctions have been used in literature. For instance Chen et al. [24] utilized p- $\mathrm{ZnO} / \mathrm{TiO}_{2}$ and reported higher photocatalytic activity compared to $\mathrm{TiO}_{2}$. Na Lian et al. [25] reported enhanced photocatalytic activity using an $\mathrm{Ag}_{2} \mathrm{O} / \mathrm{Bi}_{2} \mathrm{O}_{2} \mathrm{CO}_{3}$ p-n heterojunction. The photocatalytic results are in agreement with AFM studies. In the case of n- $\mathrm{Si} / \mathrm{TiO}_{2}$ 
and p-Si/6 $\mu \mathrm{m} \mathrm{SiO}_{2} / \mathrm{TiO}_{2}$ no significant photocatalytic activity even at long residence times was observed. This is in agreement with AFM analysis where no difference in terms of force and surface charge under illumination in n-Si/ $\mathrm{TiO}_{2}$ and $\mathrm{p}-\mathrm{Si} / \mathrm{SiO}_{2} / \mathrm{TiO}_{2}$ samples was observed.

\subsection{Conclusion}

We investigated the surface properties of $\mathrm{TiO}_{2}$ thin film patterns on different substrates (n-Si, p-Si and $\mathrm{SiO}_{2}$ ). We monitored the local surface charge density at the $\mathrm{TiO}_{2}$-electrolyte interface. We show that depending on the type of substrates, the surface charge significantly changes upon illumination. Using a microreactor we assessed the photocatalytic performance of the $\mathrm{TiO}_{2}$ on different substrates. We observed that the highest photocatalytic performance belongs to the $\mathrm{p}-\mathrm{Si} / \mathrm{TiO}_{2}$ due to enhanced charge separation which is in good agreement with AFM study. 


\section{Bibliography}

[1] M. Abdulla-Al-Mamun, Y. Kusumoto, T. Zannat, and M. S. Islam, Synergistic enhanced photocatalytic and photothermal activity of $\mathrm{Au} @ \mathrm{TiO}_{2}$ nanopellets against human epithelial carcinoma cells, Physical Chemistry Chemical Physics 13, 21026 (2011).

[2] X. He, Y. Cai, H. Zhang, and C. Liang, Photocatalytic degradation of organic pollutants with $\mathrm{Ag}$ decorated free-standing $\mathrm{TiO}_{2}$ nanotube arrays and interface electrochemical response, Journal of Materials Chemistry 21, 475 (2011).

[3] J. S. Jang, S. H. Choi, H. G. Kim, and J. S. Lee, Location and State of Pt in Platinized $\mathrm{CdS} / \mathrm{TiO}_{2}$ Photocatalysts for Hydrogen Production from Water under Visible Light, J. Phys. Chem. C 112, 17200 (2008).

[4] W. Zhou, H. Liu, J. Wang, D. Liu, G. Du, S. Han, J. Lin, and R. Wang, Interface dominated high photocatalytic properties of electrostatic self-assembled $\mathrm{Ag}_{2} \mathrm{O} / \mathrm{TiO}_{2}$ heterostructure, Physical Chemistry Chemical Physics 12, 15119 (2010).

[5] G. Li, L. Chen, M. E. Graham, and K. a. Gray, A comparison of mixed phase titania photocatalysts prepared by physical and chemical methods: The importance of the solidsolid interface, Journal of Molecular Catalysis A: Chemical 275, 30 (2007).

[6] J. Georgieva, E. Valova, S. Armyanov, N. Philippidis, I. Poulios, and S. Sotiropoulos, Bi-component semiconductor oxide photoanodes for the photoelectrocatalytic oxidation of organic solutes and vapours: A short review with emphasis to $\mathrm{TiO}_{2}-\mathrm{WO}_{3}$ photoanodes, Journal of Hazardous Materials 211-212, 30 (2012).

[7] R. Amadelli, L. Samiolo, a.B. Velichenko, V. Knysh, T. Lukyanenko, and F. Danilov, Composite $\mathrm{PbO}_{2}-\mathrm{TiO}_{2}$ materials deposited from colloidal elec- 
trolyte: Electrosynthesis, and physicochemical properties, Electrochimica Acta 54, 5239 (2009).

[8] H. Park, A. Bak, Y. Y. Ahn, J. Choi, and M. R. Hoffmannn, Photoelectrochemical performance of multi-layered $\mathrm{BiOxTiO}_{2} / \mathrm{Ti}$ electrodes for degradation of phenol and production of molecular hydrogen in water, Journal of Hazardous Materials 211-212, 47 (2012).

[9] Y.-G. Zhang, L.-L. Ma, J.-L. Li, and Y. Yu, In Situ Fenton Reagent Generated from $\mathrm{TiO}_{2} / \mathrm{Cu}_{2} \mathrm{O}$ Composite Film: a New Way to Utilize $\mathrm{TiO}_{2}$ under Visible Light Irradiation, Environmental Science \& Technology 41, 6264 (2007).

[10] C.-J. Chen, C.-H. Liao, K.-C. Hsu, Y.-T. Wu, and J. C. Wu, PN junction mechanism on improved $\mathrm{NiO} / \mathrm{TiO}_{2}$ photocatalyst, Catalysis Communications 12, 1307 (2011).

[11] K. Hu, F.-R. F. Fan, A. J. Bard, and A. C. Hillier, Direct Measurement of Diffuse Double-Layer Forces at the Semiconductor/Electrolyte Interface Using an Atomic Force Microscope, The Journal of Physical Chemistry B 101, 8298 (1997).

[12] I. Siretanu, D. Ebeling, M. P. Andersson, S. L. S. Stipp, A. Philipse, M. C. Stuart, D. van den Ende, and F. Mugele, Direct observation of ionic structure at solid-liquid interfaces: a deep look into the Stern Layer., Scientific reports 4, 4956 (2014).

[13] D. Rafieian, W. Ogieglo, T. Savenije, and R. G. H. Lammertink, Controlled formation of anatase and rutile $\mathrm{TiO}_{2}$ thin films by reactive magnetron sputtering, AIP Advances 5, 097168 (2015).

[14] C. Zhao, D. Ebeling, I. Siretanu, D. van den Ende, and F. Mugele, Extracting local surface charges and charge regulation behavior from atomic force microscopy measurements at heterogeneous solid-electrolyte interfaces, Nanoscale 7, 16298 (2015).

[15] D. Rafieian, R. T. Driessen, W. Ogieglo, and R. G. Lammertink, Intrinsic Photocatalytic Assessment of Reactively Sputtered $\mathrm{TiO}_{2}$ Films, ACS Applied Materials \& Interfaces 7, 8727 (2015).

[16] M. D. Chadwick, J. W. Goodwin, E. J. Lawson, P. D. a. Mills, and B. Vincent, Surface charge properties of colloidal titanium dioxide in ethylene 
glycol and water, Colliods. Surf. A 203, 229 (2002).

[17] J. P. Holmberg, E. Ahlberg, J. Bergenholtz, M. Hassellöv, and Z. Abbas, Surface charge and interfacial potential of titanium dioxide nanoparticles: Experimental and theoretical investigations, Journal of Colloid and Interface Science 407, 168 (2013).

[18] A. W. Bott and D. Ph, Electrochemistry of Semiconductors, Solar Energy 8, 136 (1964).

[19] R. Beranek, (Photo)electrochemical Methods for the Determination of the Band Edge Positions of $\mathrm{TiO}_{2}$-Based Nanomaterials, Advances in Physical Chemistry 2011, 1 (2011).

[20] A. Visan, D. Rafieian, W. Ogieglo, and R. G. H. Lammertink, Modeling intrinsic kinetics in immobilized photocatalytic microreactors, Applied Catalysis, B: Environmental 150-151, 93 (2014).

[21] D. O. Scanlon et al., Band alignment of rutile and anataseTiO $O_{2}$, Nature Materials 12, 798 (2013).

[22] Y. J. Hwang, A. Boukai, and P. Yang, High density n-Si/n-TiO2 core/shell nanowire arrays with enhanced photoactivity., Nano letters 9, 410 (2009).

[23] X. Zhang, Photocatalytic microreactors for water purification: Selective control of oxidation pathways, The 8th Annual IEEE International Conference on Nano/Micro Engineered and Molecular Systems 1, 368 (2013).

[24] C. Shifu, Z. Wei, L. Wei, Z. Huaye, Y. Xiaoling, and C. Yinghao, Preparation, characterization and activity evaluation of pn junction photocatalyst $p$ - $\mathrm{CaFe}_{2} \mathrm{O}_{4} / n-\mathrm{Ag}_{3} \mathrm{VO}_{4}$ under visible light irradiation, Journal of Hazardous Materials 172, 1415 (2009).

[25] N. Liang, M. Wang, L. Jin, S. Huang, W. Chen, M. Xu, Q. He, J. Zai, N. Fang, and X. Qian, Highly Efficient $\mathrm{Ag}_{2} \mathrm{O} / \mathrm{Bi}_{2} \mathrm{O}_{2} \mathrm{CO}_{3}$ p-n Heterojunction Photocatalysts with Improved Visible-Light Responsive Activity, ACS Applied Materials \& Interfaces 6, 11698 (2014). 



\section{CHAPTER 5}

\section{Porous Titanium Dioxide Thin Film; Experimental}

and Modeling Study

This chapter presents fabrication and modeling of an immobilized porous $\mathrm{TiO}_{2}$ thin film based photocatalytic microreactors. The model follows a first order reaction rate within the porous film with either light independency or light dependency described by photon absorption carrier generation semiconductor physics. Experimental data obtained for various residence times, catalyst thicknesses and photon flux densities proved that the model is capable of describing the reactor performance. The extracted reaction rate constant $k$ reveals the intrinsic kinetics as both external and internal mass transport are accounted for. The effect of light is also considered by defining a criterion for neglecting light intensity based on film thickness and absorption coefficient. For the first time $k$ values on the order of magnitude of $10^{1} 1 / \mathrm{s}$ corresponding $10^{-7} \mathrm{~m} / \mathrm{s}$ surface to volume normalized are reported. In the end, performance parameters are also derived for the light dependent model for which the internal effectiveness factor reveals both mass transfer and light limitations.

This chapter is based on: Aura Visan, Damon Rafieian, Wojciech Ogieglo, Rob G.H. Lammertink, Modeling Intrinsic Kinetics in Immobilized Photocatalytic Microreactors, Applied Catalysis B: Environmental, Volumes 150-151, 5 May 2014, Pages 93-100. 


\subsection{Introduction}

Heterogeneous photocatalysis is a promising technology for environmental remediation. However, commercial products are focused mostly on self-cleaning surfaces and air filtering. Important applications such as removing refractory compounds from wastewater are scarce. In 2007 van Gerven et al.[1] mentioned only 6 examples: Zentox Corporation, Matrix Photocatalytic Inc., Clearwater Industries, Photox Bradford Ltd., Lynntech Inc. and Purifics Environmental Technologies Inc. The invoked reasons are small conversion capacities and inefficient light use, addressing especially the inconsistencies in the definitions of various performance parameters. A progress in optimization requires that individual research efforts can be combined which is possible only if an honest comparison between them can be achieved.

One parameter used frequently when evaluating the conversion capacity of a reactor is the apparent reaction rate constant. Its derivation implies identifying the reaction rate with the conversion $-d c / d t=r$. The kinetic model is usually Langmuir-Hinshelwood $r=\frac{k K c}{1+K c}$ or a first order reaction $r=k c$ from which the reaction rate constant is obtained $[2-10]$. The agreement with respect to these basic relations is widespread, as several reviews mention it $[11,12]$.

Heterogeneous reactions involve the diffusion of species to the active catalyst sites. Mass transport becomes important and if omitted can alter the true values of the reaction kinetics. Intrinsic kinetic parameters are of paramount importance. They are required for catalyst screening and necessary when simple engineering tools such as the apparent reaction constant are used for comparing different reactor configurations. The real value for the reaction rate constant allows the evaluation of the mass transfer contribution on the conversion which is a main factor dictating the performance of a reactor. Evidently, comparison complicates further when the gradients in photon absorption become important and kinetics cannot be volume averaged anymore. When both immobilized and dispersed systems are considered, the difference in light dispersion can be bridged only by light dependent intrinsic kinetics.

The standard approach for kinetic investigation is to place the system in the reaction rate limiting regime, so the apparent reaction rate can reach the intrinsic 
value. The classical method uses a differential reactor which consists of a reaction chamber and a mixing tank, where the reaction volume is much smaller than the total volume. The small conversion per pass allows the simplification of the mass balance to a batch reactor equation. The intrinsic kinetics are determined for flowrate-independent conversions [13-24]. Accepting the reliability of this method to eliminate external mass transport, the question about internal mass transfer remains, which will be present even for thin catalyst layers or for an inevitable degree of aggregation in slurry systems. Ballari et al. [25-27] went on to defining guidelines for canceling mass transfer limitations in slurry reactors based on flow rate, catalyst loading and irradiation rates.

Even when concentration gradients are eliminated, the inhomogeneity regarding light distribution has to be carefully considered, especially for slurry systems. Motegh et al. [28] gave guidelines for operating in optically differential mode. Starting from the premise of perfect mixing, a criterion was defined for keeping the gradients in photon absorption rate small enough as to allow the volumeaveraging of the reaction rate.

A more thorough method is to model light distribution, fluid dynamics and mass transfer and fit the kinetics to the experimental data. Due to the complexity of large-scale photocatalytic reactors, a lot of assumptions come into play.

In this chapter a complete model for immobilized porous film photocatalytic microreactors is set up and solved for both light independency and light dependency first order kinetics. Experimental data obtained for various residence times, catalyst thicknesses and photon flux densities proves the model is capable of describing the reactor performance. Furthermore, a criterion is defined based on the absorption coefficient and catalyst thickness to mark the transition towards the regime where the incorporation of photon flux density is required. Performance parameters are also derived for the light dependent model for which the internal effectiveness factor reveals both mass transfer and light limitations. The potential of the models is not confined to microreactor engineering. Scale-up is straightforward compared to slurry reactors as only channel height and catalyst thickness should be designed for a specific flowrate. 


\subsection{Experimental}

\subsection{1 $\mathrm{TiO}_{2}$ immobilization}

The silicon chips diced from $4^{\prime \prime}$ wafers were cleaned with $65 \%$ nitric acid (Merck) for $15 \mathrm{~min}$ and rinsed with water and acetone. Following the cleaning, the substrates were covered entirely with commercial suspension (VP Disp. W 2730 X, Evonik) and spin coated at different angular velocities and solid content. The spin coating time was kept at $1 \mathrm{~min}$. The resulted layer was sintered for $2 \mathrm{~h}$ at $500{ }^{\circ} \mathrm{C}$ in air. The heating and cooling rates were kept at $2{ }^{\circ} \mathrm{C} / \mathrm{min}$.

\subsubsection{Microreactor fabrication}

The microreactor was assembled by attaching the $\mathrm{TiO}_{2}$ coated substrate to a PDMS slab containing the microchannel replicated from a microstructured mold. The PDMS was prepared by blending the polymer base (RTV-615 A, Permacol) with the curing agent (RTV-615 B, Permacol) in 10:1 ratio as indicated by the manufacturer. The mixture was poured on top of a patterned wafer and degassed in a vacuum desiccator. After partial curing for $45 \mathrm{~min}$ at $60^{\circ} \mathrm{C}$, while still preserving its adhesive properties, the PDMS slabs were cut out from the mold, punctured for the inlet and outlet connections, attached to the substrate containing the catalyst layer and cured for another $45 \mathrm{~min}$. To ensure a leakfree assembly, additional PDMS was poured and cured for $3 \mathrm{~h}$ at $70^{\circ} \mathrm{C}$. The inlet and outlet connections were made by introducing needles into the previously punctured holes. The preparation steps are illustrated in Fig. 5.1.

The microstructured mold was made by standard photolithography using negative photoresist (SU-8). SU-8 was spun on silicon wafer at $500 \mathrm{rpm}$ for 10 seconds and continued to $100 \mathrm{rpm}$ for 30 seconds. Then the sample was prebaked at 50, $65,95{ }^{\circ} \mathrm{C}$ for $10,10,45$ minutes respectively. This is followed by UV exposure for 33 seconds and post baking at $50,65,80{ }^{\circ} \mathrm{C}$ for $5,10,20$ minutes respectively. Finally the sample was spray developed by RER 600 . The resulted SU-8 height was measured with optical profiler. The channel is rectangular and follows a meandering path. The basic dimensions are: $50 \mu \mathrm{m}$ height, $500 \mu \mathrm{m}$ width and $5.96 \mathrm{~cm}$ length, with a total volume of $1.49 \mu \mathrm{l}$. 


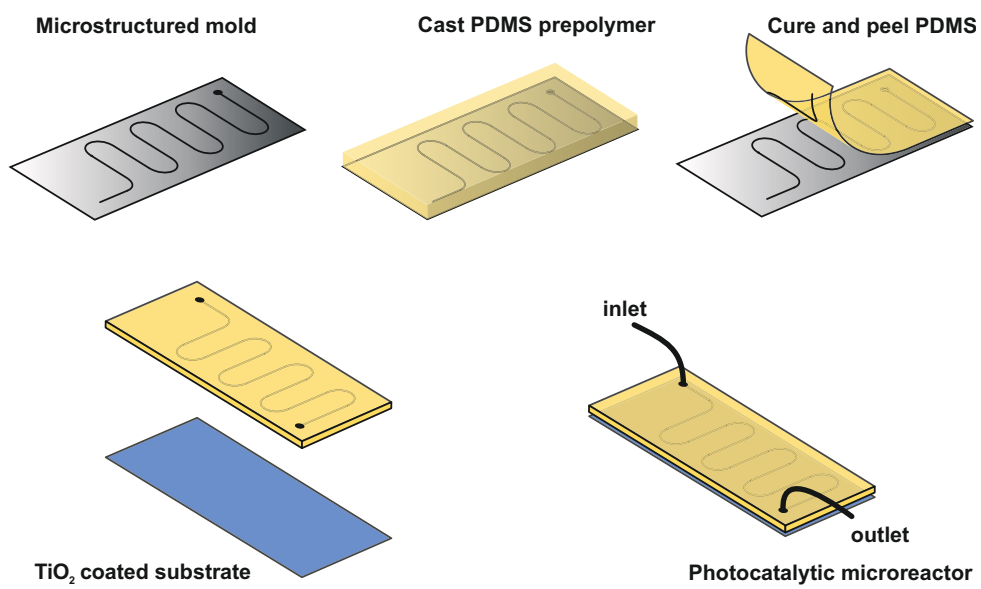

Figure 5.1: Schematic of the microreactor fabrication steps. PDMS is cast on a microstructures mold and released after curing. The PDMS slab is subsequently sealed to a $\mathrm{TiO}_{2}$ coated substrate.

Since oxygen plays an important role in photocatalytic reaction $[29,30]$ the $\mathrm{O}_{2}$ permeability of the PDMS layer was confirmed by monitoring the $\mathrm{O}_{2}$ concentration after passing $\mathrm{N}_{2}$ saturated water through the reactor. The $\mathrm{O}_{2}$ inline detector (PreSens Fibox 3 minisensor oxygen meter) recorded a steady state concentration very close to the maximum solubility of $\mathrm{O}_{2}$ in water at atmospheric pressure indicating $\mathrm{O}_{2}$ was continuously replenished through the permeable PDMS layer. In addition the transparency of PDMS at $365 \mathrm{~nm}$ wavelength was checked using a FHS-UV In-Line Filter Holder (Ocean Optics) and the UV-VIS spectrometer (USB2000+ Miniature Fiber Optic Spectrometer by Ocean Optics).

\subsubsection{Catalyst layer characterization}

High resolution SEM (Analysis Zeiss MERLIN HR-SEM) was used to investigate the morphology and thickness of the deposited thin films.

M2000-X variable angle spectroscopic ellipsometer by J.A. Woollam was used for fast and non-invasive thickness measurements. The accuracy of the method was validated by comparing one of the results with the value obtained from SEM. Porosity and roughness was extracted by ellipsometry measurements. Porosity was determined by the Bruggeman Effective Medium Approximation, EMA, [31] by mixing properties of dense $\mathrm{TiO}_{2}$ and void in a self consistent manner. Brugge- 
man type of EMA was used because it is well valid when the volume fractions of both components are comparable. Roughness was also estimated using EMA, this time adding a layer on top of the samples with assumed $50 \%$ of material and $50 \%$ of void and fitting its thickness. Values of $45 \%$ and $10 \mathrm{~nm}$, respectively, were consistent for thicknesses ranging between 300 and $4000 \mathrm{~nm}$.

Ellipsometry was also used for evaluating optical constants. Due to the homogeneity of the film, scattering was almost absent. Consequently, the model fitted very well and the accuracy of the measurement was high. For the extinction coefficient determination, we modeled the dielectric dispersion of the material by parametrization with B-Splines which is basically a polynomial spline function [32]. The credibility of this method was validated by comparing the results with the absorption coefficient computed from transmission measurements of $\mathrm{TiO}_{2}$ films of varying thickness. The transmission measurements were performed by connecting a FHS-UV In-Line Filter Holder by Ocean Optics to the UV-VIS spectrometer (USB2000+ Miniature Fiber Optic Spectrometer by Ocean Optics) where the quartz substrates coated with the catalyst film were fixed. Quartz glass was used as a substrate due to its transparency in the far UV range. Cleaned quartz substrates were used to reference the transmission. The absorption coefficient was calculated only for a single wavelength $(365 \mathrm{~nm})$ as a mere validation tool from the slope of the straight line fitting $-\ln (T / 100)$ against thickness based on the Lambert-Beer law: $T / 100=I / I_{0}=e^{-\alpha \Delta x}$. It works well when normal parametrization of the dielectric function requires too many dielectric oscillators.

\subsubsection{Microreactor operation}

An aqueous solution of $10 \mathrm{mg} / \mathrm{L}$ cortisone acetate (CA) (Sigma-Aldrich) was injected into the microreactor with flowrates of $5,10,20,30,40$ and $50 \mu \mathrm{L} / \mathrm{min}$ using a syringe pump.

The CA concentration was determined by light absorbance measurements using USB2000+ Miniature Fiber Optic Spectrometer by Ocean Optics. The monitored wavelength was $244.48 \mathrm{~nm}$, corresponding to the maximum absorption peak of CA. The calibration was carried out for different concentrations of CA solutions (from 5 to $20 \mathrm{mg} / \mathrm{L}$ ).

When adsorption reached steady state and the concentration recorded by the 


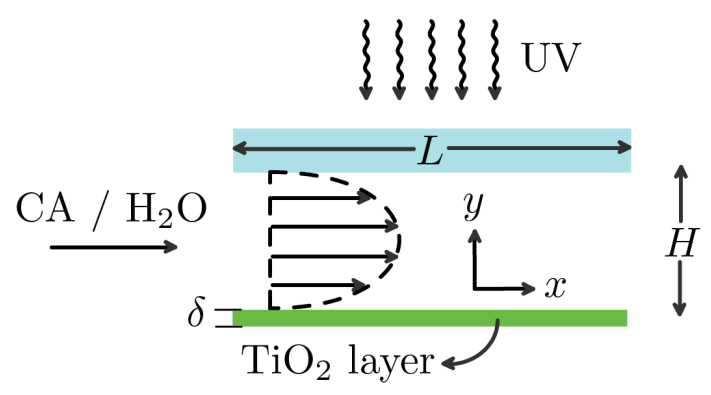

Figure 5.2: Schematic representation of the 2D model, indicating channel length $L$, channel height $H$, and catalyst thickness $\delta$

UV-VIS spectrometer showed the inlet value, the UV lamp (UV-Point Source HP-120, Dr. Gröbel UV-Electronik GmbH) was turned on. The photocatalytic degradation of CA was continuously monitored by passing the outlet stream through a flowcell (Fialab SMA Z-flowcell $10 \mathrm{~mm}$ path length) connected to the spectrometer. For the first batch of experiments, the irradiance was set at 180 $\mathrm{W} / \mathrm{m}^{2}$. For the second part, the light intensity was varied between 55 and 270 $\mathrm{W} / \mathrm{m}^{2}$ by changing the distance between the light guide and the microreactor. Due to the sharp emission peak at 365 , there was no necessity for integrating the irradiance over the whole $\mathrm{TiO}_{2}$ absorbing wavelength range. The total incoming photon flux density was approximated to the value corresponding to $365 \mathrm{~nm}$ wavelength which was measured using an optical power meter (Newport 1916$\mathrm{R})$.

\subsection{Model}

\subsubsection{Light independent model}

Fig. 5.2 illustrates the schematics of the model. The reactor was divided in 2 domains: the flow channel and the catalyst film.

The governing equation in the catalyst layer includes diffusion and reaction only. The reaction rate is assumed to be first order with respect to the cortisone acetate, zero order with respect to oxygen due its provision through the permeable PDMS layer and zero order with respect to irradiance. Hence, the mass balance 
for the catalyst layer reduces to:

$$
D_{e f f} \frac{\partial^{2} c}{\partial y^{2}}-k c=0
$$

Where $D_{\text {eff }}=D \frac{\epsilon}{\tau}$ is the effective diffusion coefficient, $D$ is the molecular diffusion coefficient for cortisone acetate in water, $\epsilon$ is the porosity, $\tau$ is the tortuosity and $k$ is the first order reaction rate constant.

The bottom boundary for the catalyst layer has the flux equal to zero:

$$
y=\delta: D_{\text {eff }} \frac{\partial c}{\partial y}=0
$$

The boundary condition at the interface between the channel and the catalyst is represented by flux and concentration continuity:

$$
y=0: c=c_{L}, D_{e f f} \frac{\partial c}{\partial y}=D \frac{\partial c_{L}}{\partial y}
$$

The diffusion reaction equation together with the boundaries mentioned above have the following analytical solution:

$$
c(y)=\frac{\cosh \left(\frac{\phi y}{\delta}\right)+\cosh \left(\phi\left(2-\frac{y}{\delta}\right)\right)}{\cosh (2 \phi)+1}
$$

where Thiele modulus is $\phi=\sqrt{\frac{k}{D_{\text {eff }}}} \delta$.

Hence, the domain of the catalyst layer can be collapsed into a single flux boundary condition for the flow channel:

$$
N_{y=0}=\frac{-D_{e f f} \phi c_{L}}{\delta} \tanh (\phi)
$$

The transport of the model compound in the flow channel is governed by advection and diffusion.

$$
u(y) \frac{\partial c_{L}}{\partial x}=D \frac{\partial^{2} c_{L}}{\partial y^{2}}
$$

where $u(y)=u_{a v g}\left(-6 \frac{y^{2}}{H^{2}}+6 \frac{y}{H}\right)$ represents a parabolic velocity profile.

The governing equation for the flow channel was non-dimensionalized to a square $1 \times 1$ domain (eq. 5.7) together with the boundary conditions (eq. 5.8 
and 5.9) and numerically solved in Matlab.

$$
u_{a v g}\left(-6 y^{\prime 2}+6 y^{\prime}\right) \frac{1}{L} \frac{H^{2}}{D} \frac{\partial c^{\prime}}{\partial x^{\prime}}=\frac{\partial^{2} c^{\prime}}{\partial y^{\prime 2}}
$$

where $x^{\prime}=x / L, y^{\prime}=y / H, c^{\prime}=c_{L} / c_{0}$.

$$
N_{y^{\prime}=0}=\frac{-D_{e f f} \phi c^{\prime} H}{\delta} \tanh (\phi)
$$

for the boundary to the catalytic coating.

$$
N_{y^{\prime}=1}=D \frac{\partial c^{\prime}}{\partial y^{\prime}}=0
$$

for the upper wall.

\subsubsection{Light dependent model}

To account for light dependent conversion, the photon flux density was incorporated in the reaction rate described by photon absorption carrier generation first order kinetics. The expression was taken form Nielsen et al. [33], derived based on semiconductor physics. The governing equation for the catalyst layer becomes:

$$
D_{e f f} \frac{\partial^{2} c}{\partial y^{2}}-k c\left(\frac{\alpha \Phi_{0}}{B p_{0} n_{0} \hbar \omega}\right)^{\gamma} \exp (-\gamma \alpha y)=0
$$

where $B p_{0} n_{0} \cong 3.3 \times 10^{-23} m^{-3} s^{-1}$ is the equilibrium electron-hole recombination rate [33], $\Phi_{0}$ is the incoming photon flux density, $\hbar \omega$ is the photon energy, $\gamma$ is the transfer coefficient for the electron transfer process and $\alpha$ is the absorption coefficient.

Given that the boundary conditions remain the same, the new analytical solution for the concentration profile in the catalyst layer becomes:

$$
c(y)=c_{L} \frac{I_{1}\left(\frac{2 \phi \sqrt{e^{-\gamma \alpha \delta}}}{\gamma \alpha \delta}\right) K_{0}\left(\frac{2 \phi \sqrt{e^{-\gamma \alpha y}}}{\gamma \alpha \delta}\right)+K_{1}\left(\frac{2 \phi \sqrt{e^{-\gamma \alpha \delta}}}{\gamma \alpha \delta}\right) I_{0}\left(\frac{2 \phi \sqrt{e^{-\gamma \alpha y}}}{\gamma \alpha \delta}\right)}{I_{0}\left(\frac{2 \phi}{\gamma \alpha \delta}\right) K_{1}\left(\frac{2 \phi \sqrt{e^{-\gamma \alpha \delta}}}{\gamma \alpha \delta}\right)+K_{0}\left(\frac{2 \phi}{\gamma \alpha \delta}\right) I_{1}\left(\frac{2 \phi \sqrt{e^{-\gamma \alpha \delta}}}{\gamma \alpha \delta}\right)}
$$


where $\phi^{2}=\frac{k_{i} \delta^{2}}{D_{\text {eff }}}\left(\frac{\alpha \Phi_{0}}{B p_{0} n_{0} \hbar \omega}\right)^{\gamma}$ and $I_{n}(x)$ and $K_{n}(x)$ are modified Bessel functions of the first and second kind.

This translates in the following boundary condition for the flow channel, giving the flux towards the catalyst layer which is again non-dimensionalized and implemented in Matlab:

$$
N_{y^{\prime}=0}=\frac{-D_{e f f} \phi H c^{\prime}}{\delta} \frac{I_{1}\left(\frac{2 \phi \sqrt{e^{-\gamma \alpha \delta}}}{\gamma \alpha \delta}\right) K_{1}\left(\frac{2 \phi}{\gamma \alpha \delta}\right)-K_{1}\left(\frac{2 \phi \sqrt{e^{-\gamma \alpha \delta}}}{\gamma \alpha \delta}\right) I_{1}\left(\frac{2 \phi}{\gamma \alpha \delta}\right)}{I_{0}\left(\frac{2 \phi}{\gamma \alpha \delta}\right) K_{1}\left(\frac{2 \phi \sqrt{e^{-\gamma \alpha \delta}}}{\gamma \alpha \delta}\right)+K_{0}\left(\frac{2 \phi}{\gamma \alpha \delta}\right) I_{1}\left(\frac{2 \phi \sqrt{e^{-\gamma \alpha \delta}}}{\gamma \alpha \delta}\right)}
$$

Note that the governing equation is the same for both the light dependent and light independent model, and that the two models only differ in the boundary condition describing the flux into the catalyst layer.

The geometry of the channel was simplified to a rectangle preserving the height, $H$, of $50 \mu \mathrm{m}$ and the length, $L$, of $5.96 \mathrm{~cm}$. The mesh was refined such that mesh independent results were obtained.

\subsection{Results and Discussion}

\subsubsection{Film preparation}

High resolution SEM (figure 5.3) shows a highly homogeneous film with a narrow particle size distribution around $(20 \mathrm{~nm})$ with uniform porosity. throughout the layer. The morphology of layers is reproducible due to the same spin coating parameters used during preparation. In order to modify the thickness of the $\mathrm{TiO}_{2}$ layers the angular velocity and solid content of the suspension were adjusted. Figure 5.4 (a) shows the thickness of the $\mathrm{TiO}_{2}$ as a function of spincoating angular velocity which was varied between 1000 and $8000 \mathrm{rpm}$. At high velocities the thickness becomes constant. As the velocity increases, the variation between the thicknesses at the center and the edges of the film becomes more obvious. Hence, the optimum velocity is at the lower limit of the plateau, around $3000 \mathrm{rpm}$.

The viscosity of the $\mathrm{TiO}_{2}$ suspension has a strong connection to the final thickness of the spin coated film. The complex rheological properties of a nanoparticle 
suspension suggested that experimental data would be more reliable to relate the solid content to the viscosity than equations such as Einstein's [34], Mooney's [35], Krieger-Dougherty's [36]. According to Tseng and Lin the viscosity of the suspension has an exponential increase with the volumetric solid content, suggesting that the solid content will strongly affect the film thickness [37]. Thicknesses between 300 and $1200 \mathrm{~nm}$ were achieved by solid content modification (figure 5.4 (b)). In order to increase the thickness beyond $1200 \mathrm{~nm}$, multiple coatings with sintering in between were applied. The result was successful given the fact that the final thickness of the $\mathrm{TiO}_{2}$ layer was multiplied by the number of coatings.

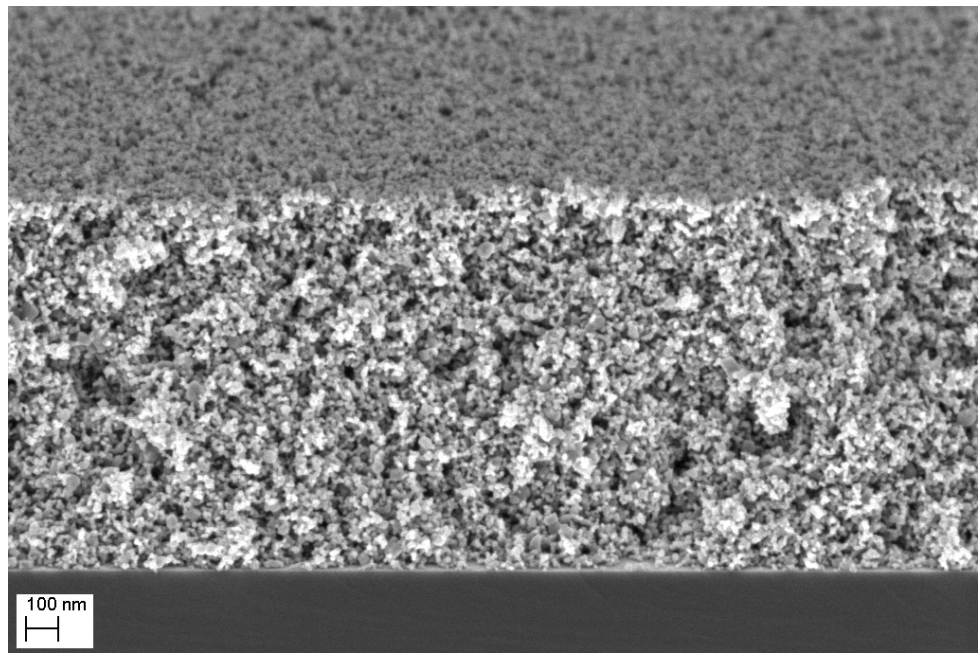

Figure 5.3: SEM cross-section of a porous $\mathrm{TiO}_{2}$ film.

Figure 5.5 shows the absorption coefficient of the $\mathrm{TiO}_{2}$ film obtained by ellipsometry. The extinction coefficient is converted to absorption coefficient using the formula: $\alpha=4 \pi \beta / \lambda$ where $\beta$ is the extinction coefficient, $\alpha$ is the absorption coefficient $(1 / \mathrm{nm})$ and $\lambda$ is the wavelength $(\mathrm{nm})$. 


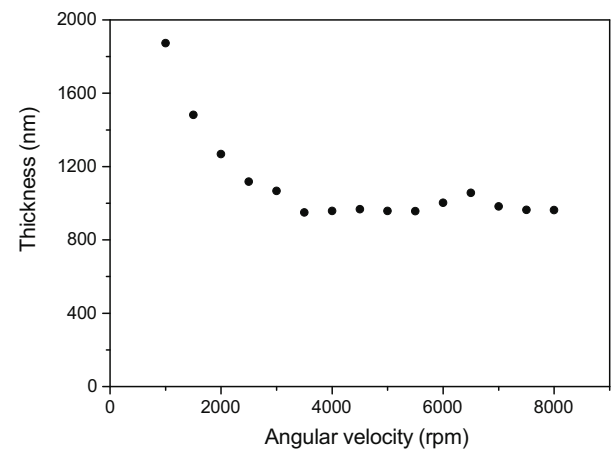

a)

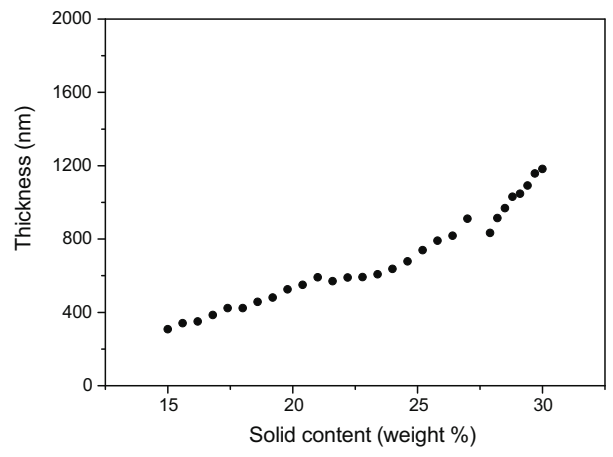

b)

Figure 5.4: The thickness of the $\mathrm{TiO}_{2}$ layer as a function of angular velocity (30 wt\%) (a) and suspension solid content (3000 rpm) (b).

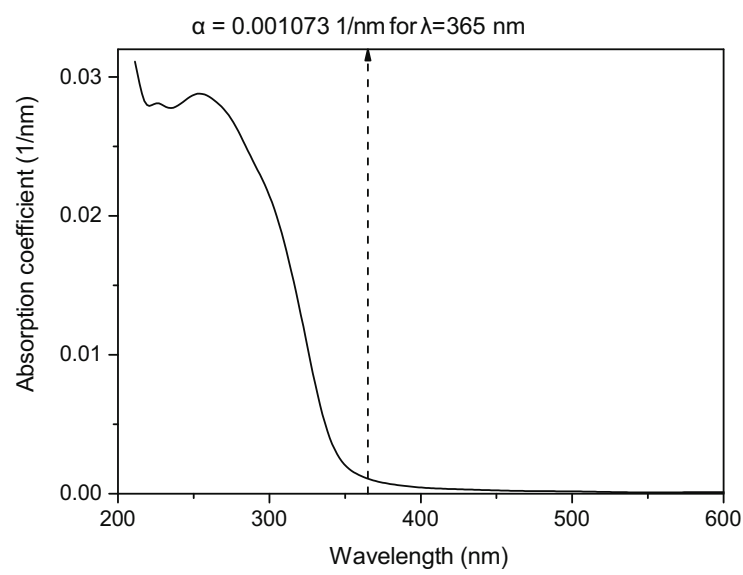

Figure 5.5: Spectral absorption coefficient of a $\mathrm{TiO}_{2}$ layer obtained by ellipsometry. The arrow shows the absorption coefficient at $365 \mathrm{~nm}$

Figure 5.6 shows transmission obtained by the $\mathrm{TiO}_{2}$ film at different thickness ranging from 310 to $1025 \mathrm{~nm}$. The insert shows the absorption coefficient for a single wavelength of $365 \mathrm{~nm}$ obtained from the slope of the straight line fitting $-\ln (T / 100)$ against thickness based on the Lambert-Beer law: $T / 100=I / I_{0}=$ $e^{-\alpha \Delta x}$. The absorption coefficient (slope) obtained by transmission measurement (1.04 $1 / \mu \mathrm{m})$ matched very well with the one obtained from the ellipsometry measurement $(1.071 / \mu \mathrm{m})$. 


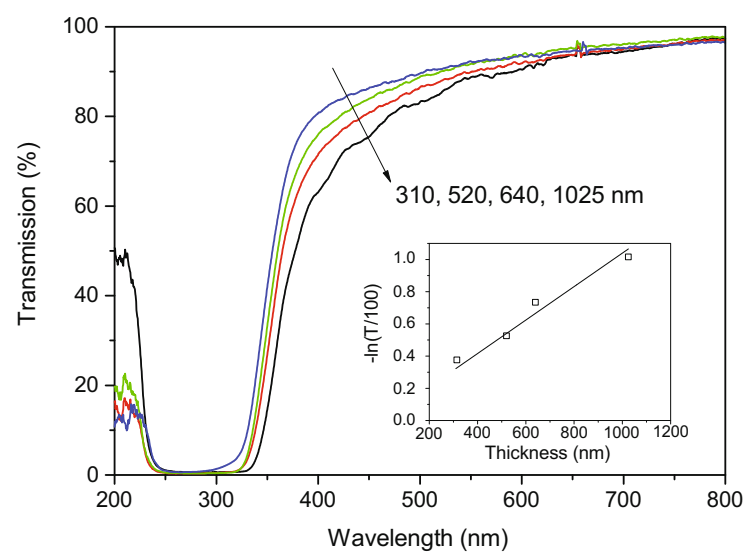

Figure 5.6: The transmisson of different thickness of $\mathrm{TiO}_{2}$ layer $(310-1025 \mathrm{~nm})$. The insert shows linear fit of $-\ln (T / 100)$ against thickness at $365 \mathrm{~nm}$.

\subsubsection{Light independent model (LIM)}

The assumption for a first order reaction rate was first justified by evaluating the effect of different residence times ranging between 1.8 and $18 \mathrm{~s}$. The degradation vs. residence time curve shown in Fig. 5.7 corresponds to $2050 \mathrm{~nm}$ catalyst thickness in order to capture the complete spectrum for the residence time range used during the experiments.

$k=311 / \mathrm{s}$ was extracted by fitting the model for the lowest residence time where experimental data is more reliable. The reaction rate constant values determined for various catalyst thicknesses ranged between 21 and 43 1/s. Their corresponding degradation curves were also plotted in Fig. 5.7. The standard deviation for the degradation is around $\pm 2.5 \%$.

The consistency of $k$ is set out in Fig. 5.8 where degradation is plotted against catalyst thickness ranging between $300 \mathrm{~nm}$ and $4000 \mathrm{~nm}$ for multiple residence times. An optimum catalyst thickness can already be deduced given the fact that an increase in thickness beyond $2000 \mathrm{~nm}$ affects the degradation only slightly.

The intrinsic $k$ values are several orders of magnitude higher than the apparent first order reaction rate constants reported in the literature. For example, for methylene blue (MB), a common model compound, values between $10^{-5}$ and $10^{-2}$ $1 / \mathrm{s}$ are usually assigned $[3,10,38-50]$. The values increase for smaller reactor scales, reaching approximately $0.31 / \mathrm{s}$ for the present study. Following the same 


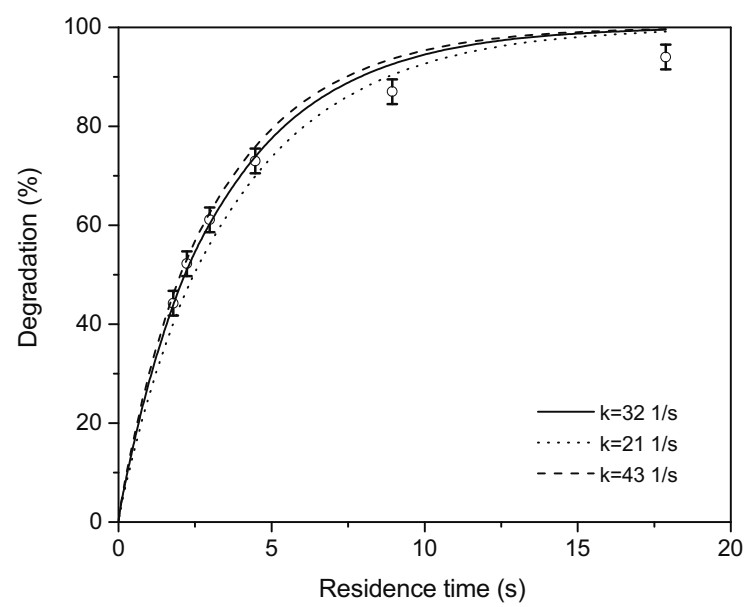

Figure 5.7: Degradation of Cortisone acetate (CA) vs. residence time for $2050 \mathrm{~nm}$ catalyst layer

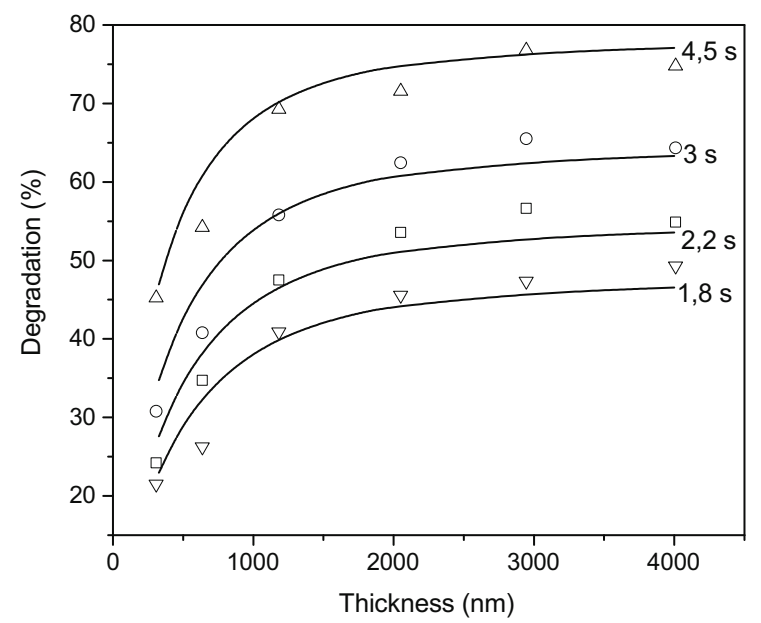

Figure 5.8: Degradation vs. thickness for multiple residence times. Symbols depict experimental results and lines correspond to the light independent model with $k=32$ $1 / \mathrm{s}$

experimental routine and simulation procedure, given the molecular diffusion coefficient for MB in water, $D=5.7 \times 10^{-10} \mathrm{~m}^{2} / \mathrm{s}$, a value of $k=401 / \mathrm{s}$ was fitted. The difference of two orders of magnitude between the real and apparent reaction rate constant draws attention on the importance of accurate modeling. The intrinsic value for the reaction rate constant allows catalyst comparison, as well as the evaluation of mass transfer for different reactor configurations. 
The use for the extracted reaction rate constant can also be extended to different catalyst densities or dispersed systems by expressing it per unit surface area. The conversion from $k$ per unit volume $[1 / \mathrm{s}]$ to $k^{\prime \prime}$ per units surface area $[\mathrm{m} / \mathrm{s}]$ has the known formula: $k^{\prime \prime}=\frac{k}{S_{a} \rho_{c}(1-\epsilon)}$, where $S_{a}=9 \times 10^{4} \mathrm{~m}^{2} / \mathrm{kg}$ is the specific surface area, $\rho_{c}=3895 \mathrm{~kg} / \mathrm{m}^{3}$ is the anatase density and $\epsilon=0.45$ is the porosity. Consequently, MB has $k^{\prime \prime}=2.07 \times 10^{-7} \mathrm{~m} / \mathrm{s}$ corresponding to $k=40$ $1 / \mathrm{s}$, while CA has $k^{\prime \prime}=1.66 \times 10^{-7} \mathrm{~m} / \mathrm{s}$ corresponding to $k=321 / \mathrm{s}$.

Not to forget, the use of light independent kinetics is valid when the reaction rate can be volume averaged with respect to the field of local volumetric rate of photon absorption. The case of immobilized systems will be discussed later by comparing the light independent with the light dependent model.

For a more quantitative optimization, the diffusion/reaction limiting regimes were investigated by evaluating the effect of $k$ on degradation, in terms of Thiele modulus. For a first order reaction occurring in a planar porous catalyst, the Thiele modulus can be computed using the following formula: $\phi=\sqrt{\frac{k}{D_{\text {eff }}}} \delta$. The overall degradation given by a $2050 \mathrm{~nm}$ catalyst film was plotted in Fig. 5.9 against Thiele modulus for $k$ ranging between $10^{-3}$ and $10^{4} 1 / \mathrm{s}$.

As $k$ and, consequently, Thiele modulus start to increase, the intrinsic chemical reaction rate starts to compete with the given diffusion rate. For the experimentally determined kinetics, $k=321 / \mathrm{s}, D_{\text {eff }}=1.4 \times 10^{-10} \mathrm{~m}^{2} / \mathrm{s}$ and $\delta=2050$ $\mathrm{nm}$, Thiele modulus is 0.98 .

We will later analyze the effectiveness of the catalyst according to this model, combined with the light dependent model.

\subsubsection{Light dependent model (LDM)}

An important part in designing a photocatalytic reactor is related to light intensity distribution as effective use of light has a big impact on the economics of the process.

Whenever kinetics are modeled with respect to light intensity, $I$, the reaction rate is considered to follow a power law: $r=b I^{\gamma}$. Based on semiconductor physics, the physical meaning of $\gamma$ would be the transfer coefficient of the electron transfer process. There is a general misunderstanding about $\gamma$, which is usually confused with the apparent $\gamma$ extracted by fitting the conversion against the 


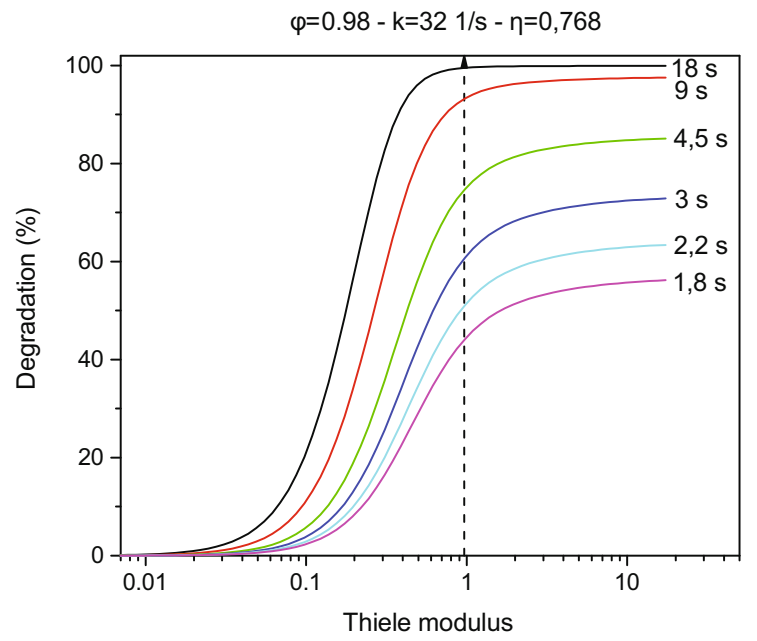

Figure 5.9: Degradation by $2050 \mathrm{~nm}$ catalyst layer vs. Thiele modulus for multiple residence times

irradiance in a power law fit.

The real decrease in $\gamma$ given by the higher enhancement in the recombination rate in connection with the excess hole production rate was found to happen at light intensities higher than $250 \mathrm{~W} / \mathrm{m}^{2}$ [51]. Moreover, values lower than 1 would translate in decreased efficiency regarding the use of light, rendering this operating range with less practical value.

The model was evaluated for the effect of different incoming photon flux densities. Fig. 5.10 shows the degradation by the $2050 \mathrm{~nm}$ catalyst film plotted against irradiance for values ranging between 55 and $270 \mathrm{~W} / \mathrm{m}^{2}$.

For the light dependent reaction rate, $r=k_{i}\left(\frac{\alpha \Phi_{0}}{B p_{0} n_{0} \hbar \omega}\right)^{\gamma} \exp (-\gamma \alpha y) c$, the constant $k_{i}$ was extracted by fitting the model to the experimental data for the higher light intensities. In this range, degradation is almost independent on the light intensity. The small order of magnitude $k_{i} \cong 10^{-48} 1 / \mathrm{s}$ comes from the high value of the prefactor $\left(\frac{\alpha \Phi_{0}}{B p_{0} n_{0} \hbar \omega}\right)^{\gamma} \cong 10^{49}$.

The challenge with the incorporation of light into the kinetic model does not come only from the increased difficulty in mathematical manipulation. The most important disadvantage is the high number of unknowns that come into play when attempting to understand the photon absorption carrier generation mechanism. Simple engineering tools are very important for reactor design and optimization. 


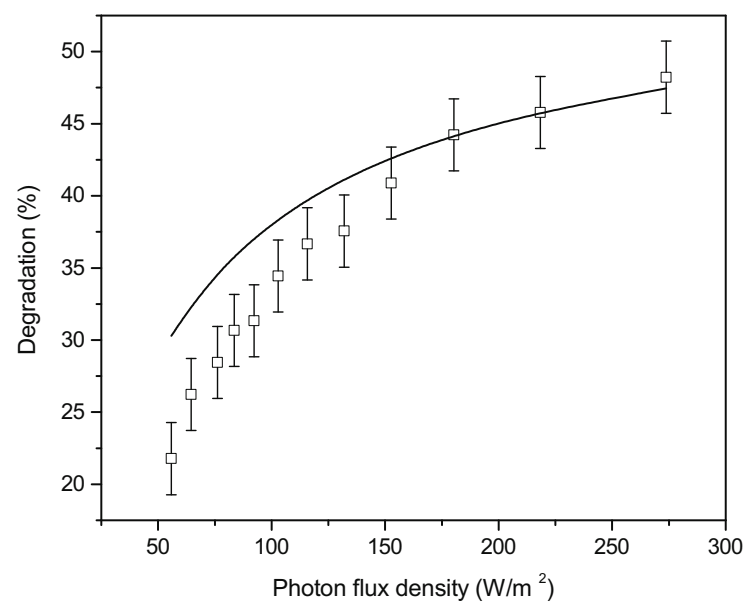

Figure 5.10: Degradation by $2050 \mathrm{~nm}$ catalyst layer vs. photon flux density. $k_{i}=$ $6.46 \times 10^{-48} 1 / \mathrm{s}, \alpha=1.073 \times 10^{6} 1 / \mathrm{m}, \gamma=1$

A simplistic model could pave the way towards a consensus regarding photocatalytic reactor design and optimization and speed up the industrial implementation. Hence, a thorough comparison between the two models was performed in order to identify the regime where the simplistic model is an admissible approximation.

\subsubsection{Criteria for neglecting light intensity}

Previously, $k$ was averaged for multiple catalyst thicknesses. However, when light is modeled, $k$ is no longer constant throughout the thickness and follows an exponential decay: $k(y)=k_{i}\left(\frac{\alpha \Phi_{0}}{B p_{0} n_{0} \hbar \omega}\right)^{\gamma} \exp (-\gamma \alpha y)$. We can define a characteristic decay length: $\delta=1 / \gamma \alpha=932.3 \mathrm{~nm}$ where the normalized $k(y)$, $k^{\prime}(y)=\exp (-\gamma \alpha y)$ decreases to $1 / e$ of its initial value. This light decay length $(\mathrm{LDL})$ is very useful in order to point out the transition towards the regime where light incorporation is recommended.

The significance of LDL can be visualized by plotting the normalized concentration profiles for both models shown in Fig. 5.11. To have a common starting point $k_{y=0}=k_{i}\left(\frac{\alpha \Phi_{0}}{B p_{0} n_{0} \hbar \omega}\right)^{\gamma}=69.4851 / \mathrm{s}$. For thicknesses higher than LDL, the concentration profiles of the two models become significantly distinct. 


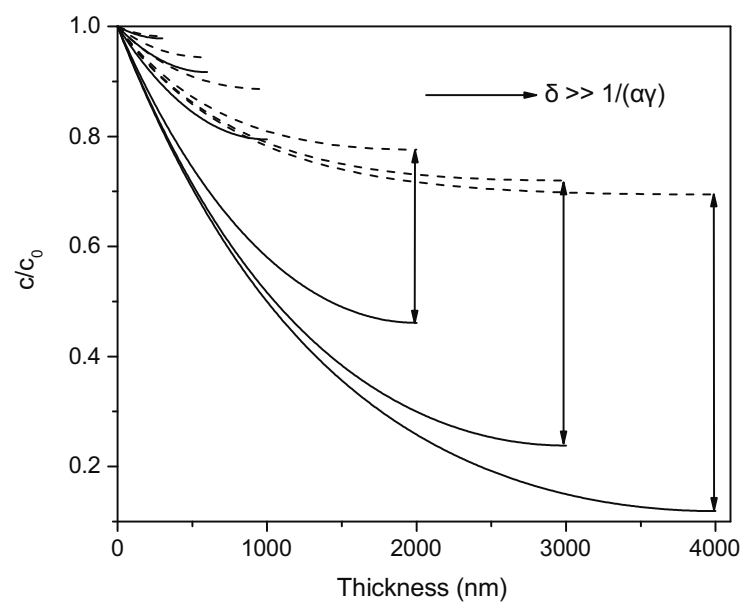

Figure 5.11: Concentration profiles vs. catalyst thickness. $k=69.4851 / \mathrm{s}$ for LIM, $k_{i}=6.46 \times 10^{-48} 1 / \mathrm{s}, \alpha=1.073 \times 10^{6} 1 / \mathrm{m}, \gamma=1, \Phi_{0}=180 \mathrm{~W} / \mathrm{m}^{2}$ for LDM

\subsubsection{Updated performance parameters}

A conclusive discussion has to include performance parameter investigation, as the efficient use of catalyst is crucial to the design procedure. Given the first order reaction in the planar porous catalyst for the LIM, the internal effectiveness factor has the well known expression: $\eta=\frac{\tanh \phi}{\phi}$, where $\phi=\sqrt{\frac{k}{D_{\text {eff }}}} \delta$. In the case of LDM, the expressions were derived based on definitions. The internal effectiveness factor represents the ratio between the actual reaction rate and the rate in the absence of concentration gradients. However, an easier way to find this is to use the flux at the surface of the catalyst which equals to the total reaction occurring inside. The internal effectiveness factor can then be expressed as:

$$
\eta=\frac{-1}{\delta} \frac{I_{1}\left(\frac{2 \phi \sqrt{e^{-\gamma \alpha \delta}}}{\gamma \alpha \delta}\right) K_{1}\left(\frac{2 \phi}{\gamma \alpha \delta}\right)-K_{1}\left(\frac{2 \phi \sqrt{e^{-\gamma \alpha \delta}}}{\gamma \alpha \delta}\right) I_{1}\left(\frac{2 \phi}{\gamma \alpha \delta}\right)}{I_{0}\left(\frac{2 \phi}{\gamma \alpha \delta}\right) K_{1}\left(\frac{2 \phi \sqrt{e^{-\gamma \alpha \delta}}}{\gamma \alpha \delta}\right)+K_{0}\left(\frac{2 \phi}{\gamma \alpha \delta}\right) I_{1}\left(\frac{2 \phi \sqrt{e^{-\gamma \alpha \delta}}}{\gamma \alpha \delta}\right)}
$$

where the updated Thiele modulus was computed as the ratio between surface reaction rate and the diffusion rate:

$$
\phi^{2}=\frac{k_{i} \delta^{2}}{D_{e f f}}\left(\frac{\alpha \Phi_{0}}{B p_{0} n_{0} \hbar \omega}\right)^{\gamma}
$$




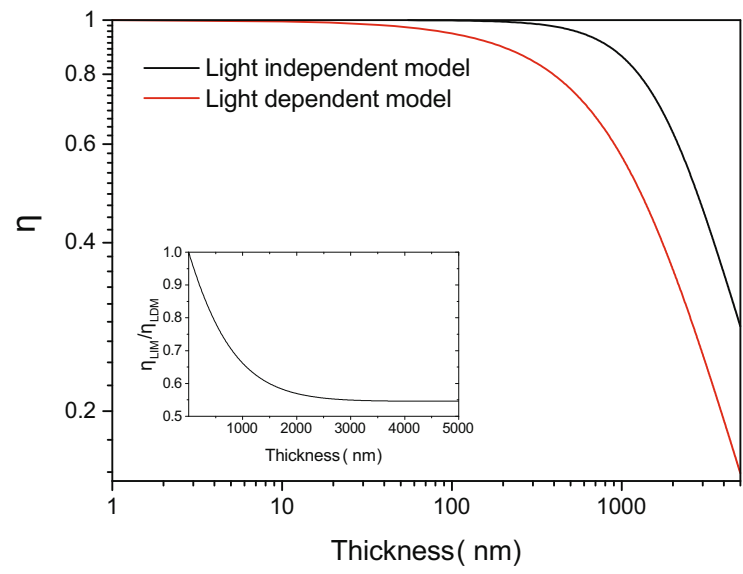

Figure 5.12: Internal effectiveness factor vs. catalyst thickness. $k=69.4851 / \mathrm{s}$ for LIM, $k_{i}=6.46 \times 10^{-48} 1 / \mathrm{s}, \alpha=1.073 \times 10^{6} 1 / \mathrm{m}, \gamma=1, \Phi_{0}=180 \mathrm{~W} / \mathrm{m}^{2}$ for $\mathrm{LDM}$

The internal effectiveness factor is plotted for both models in Fig. 5.12. $\eta_{L I M}$ accounts only for mass transfer limitation, while $\eta_{L D M}$ considers both diffusion and radiative transport when assessing catalyst coverage. The relative effectiveness factor is an indication for which transport is limiting. While in the top layer light and consequently, reaction rate are controlling, when the relative effectiveness factor levels off, mass transfer takes over and no further improvement in conversion can be sought. It can be noticed that the thickness corresponding to the transition between the two regimes is the same for which conversion reached its maximum in Fig. 5.8. Hence, the relative effectiveness factor can be considered the absolute performance parameter for immobilized photocatalytic reactors.

The maximum conversion thickness depends on the absorption coefficient and incoming photon flux density. For higher $\alpha$, the initial decrease becomes steeper, lowering the maximum conversion thickness. For higher light intensities, the maximum conversion thickness is also decreasing due to the higher $k_{y=0}$. The difference is in the asymptotic value for the relative effectiveness factor which suggests how far away the two models are from each other. For higher $\alpha$ the discrepancy between the two models is increasing, while for higher light intensities it is decreasing. 


\subsection{Conclusions}

Accurate modeling for microreactors can be achieved by applying basic physical mechanisms. This leads to a rational reactor design and easy optimization. The present chapter attempts a complete model for immobilized photocatalytic microreactors and explores their potential as investigation tools for intrinsic kinetics.

Models capable of describing reactor performance were build for a first order reaction rate with either light independency or light dependency described by photon absorption carrier generation mechanism. The extracted reaction rate constant reveals the intrinsic kinetics as both external and internal mass transport are accounted for. For the first time $k$ values on the order of magnitude $10^{1} 1 / \mathrm{s}$ are reported.

The simplification to the light independent model is justified by defining a criterion for neglecting light intensity based on film thickness and absorption coefficient. Performance parameters are also derived for the situation when irradiance has to be considered. The updated internal effectiveness factor reveals both mass transfer and light limitations. 


\section{Bibliography}

[1] T. V. Gerven, G. Mul, J. Moulijn, and A. Stankiewicz, A review of intensification of photocatalytic processes, Chemical Engineering and Processing: Process Intensification 46, 781 (2007).

[2] S. B. Kim and S. C. Hong, Kinetic study for photocatalytic degradation of volatile organic compounds in air using thin film $\mathrm{TiO}_{2}$ photocatalyst, Applied Catalysis B: Environmental 35, 305 (2002).

[3] K. Natarajan, T. S. Natarajan, H. Bajaj, and R. J. Tayade, Photocatalytic reactor based on uv-led/TiO 2 coated quartz tube for degradation of dyes, Chemical Engineering Journal 178, 40 (2011).

[4] H. Yang, G. Li, T. An, Y. Gao, and J. Fu, Photocatalytic degradation kinetics and mechanism of environmental pharmaceuticals in aqueous suspension of $\mathrm{TiO}_{2}$ : A case of sulfa drugs, Catalysis Today 153, 200 (2010).

[5] G. Plantard, F. Correia, and V. Goetz, Kinetic and efficiency of $\mathrm{TiO}_{2}$-coated on foam or tissue and $\mathrm{TiO}_{2}$-suspension in a photocatalytic reactor applied to the degradation of the 2,4-dichlorophenol, Journal of Photochemistry and Photobiology A: Chemistry 222, 111 (2011).

[6] F. Shahrezaei, Y. Mansouri, A. A. L. Zinatizadeh, and A. Akhbari, Process modeling and kinetic evaluation of petroleum refinery wastewater treatment in a photocatalytic reactor using $\mathrm{TiO}_{2}$ nanoparticles, Powder Technology 221, 203 (2012).

[7] G. S. Shephard, S. Stockenstram, D. de Villiers, W. J. Engelbrecht, and G. F. Wessels, Degradation of microcystin toxins in a falling film photocatalytic reactor with immobilized titanium dioxide catalyst, Water Research 36, 140 (2002).

[8] R. Wang, D. Ren, S. Xia, Y. Zhang, and J. Zhao, Photocatalytic degradation of bisphenol a (bpa) using immobilized $\mathrm{TiO}_{2}$ and uv illumination in a hor- 
izontal circulating bed photocatalytic reactor (hcbpr), Journal of Hazardous Materials 169, 926 (2009).

[9] W.-Y. Wang, A. Irawan, and Y. Ku, Photocatalytic degradation of acid red 4 using a titanium dioxide membrane supported on a porous ceramic tube, Water Research 42, 4725 (2008).

[10] Z. Zhang, H. Wu, Y. Yuan, Y. Fang, and L. Jin, Development of a novel capillary array photocatalytic reactor and application for degradation of azo dye, Chemical Engineering Journal 184, 9 (2012).

[11] S. Ahmed, M. Rasul, R. Brown, and M. Hashib, Influence of parameters on the heterogeneous photocatalytic degradation of pesticides and phenolic contaminants in wastewater: A short review, Journal of Environmental Management 92, 311 (2011).

[12] M. N. Chong, B. Jin, C. W. Chow, and C. Saint, Recent developments in photocatalytic water treatment technology: A review, Water Research 44, 2997 (2010).

[13] M. L. Satuf, M. J. Pierrestegui, L. Rossini, R. J. Brandi, and O. M. Alfano, Kinetic modeling of azo dyes photocatalytic degradation in aqueous $\mathrm{TiO}_{2}$ suspensions. Toxicity and biodegradability evaluation, Catalysis Today 161, 121 (2011).

[14] M. L. Satuf, R. J. Brandi, A. E. Cassano, and O. M. Alfano, Scaling-up of slurry reactors for the photocatalytic degradation of 4-chlorophenol, Catalysis Today 129, 110 (2007).

[15] C. Passalía, O. M. Alfano, and R. J. Brandi, A methodology for modeling photocatalytic reactors for indoor pollution control using previously estimated kinetic parameters, Journal of Hazardous Materials 211-212, 357 (2012).

[16] J. Marugan, R. van Grieken, C. Pablos, M. L. Satuf, A. E. Cassano, and O. M. Alfano, Modeling of a bench-scale photocatalytic reactor for water disinfection from laboratory-scale kinetic data, Chemical Engineering Journal 224, 39 (2013).

[17] J. Marugán, R. van Grieken, A. E. Cassano, and O. M. Alfano, Scaling-up of slurry reactors for the photocatalytic oxidation of cyanide with $\mathrm{TiO}_{2}$ and silica-supported $\mathrm{TiO}_{2}$ suspensions, Catalysis Today 144, 87 (2009).

[18] J. Marugán, R. van Grieken, A. E. Cassano, and O. M. Alfano, Intrinsic ki- 
netic modeling with explicit radiation absorption effects of the photocatalytic oxidation of cyanide with $\mathrm{TiO}_{2}$ and silica-supported $\mathrm{TiO}_{2}$ suspensions, Applied Catalysis B: Environmental 85, 48 (2008).

[19] N. Doucet, O. Zahraa, and M. Bouchy, Kinetics of the photocatalytic degradation of benzene, Catalysis Today 122, 168 (2007).

[20] J. E. Duran, M. Mohseni, and F. Taghipour, Modeling of annular reactors with surface reaction using computational fluid dynamics (cfd), Chemical Engineering Science 65, 1201 (2010).

[21] M. Mohseni and F. Taghipour, Experimental and CFD analysis of photocatalytic gas phase vinyl chloride (VC) oxidation, Chemical Engineering Science 59, 1601 (2004).

[22] J. Moreira, B. Serrano, A. Ortiz, and H. de Lasa, A unified kinetic model for phenol photocatalytic degradation over TiO 2 photocatalysts, Chemical Engineering Science 78, 186 (2012).

[23] M. A. Mueses, F. Machuca-Martinez, and G. Li Puma, Effective quantum yield and reaction rate model for evaluation of photocatalytic degradation of water contaminants in heterogeneous pilot-scale solar photoreactors, Chemical Engineering Journal 215-216, 937 (2013).

[24] R. L. Pozzo, R. J. Brandi, A. E. Cassano, and M. a. Baltanás, Photocatalytic oxidation of oxalic acid in dilute aqueous solution, in a fully illuminated fluidized bed reactor, Chemical Engineering Science 65, 1345 (2010).

[25] M. de los Milagros Ballari, R. Brandi, O. Alfano, and A. Cassano, Mass transfer limitations in photocatalytic reactors employing titanium dioxide suspensions: Ii. external and internal particle constrains for the reaction, Chemical Engineering Journal 136, 242 (2008).

[26] M. de los Milagros Ballari, R. Brandi, O. Alfano, and A. Cassano, Mass transfer limitations in photocatalytic reactors employing titanium dioxide suspensions: I. concentration profiles in the bulk, Chemical Engineering Journal 136, 50 (2008).

[27] M. de los Milagros Ballari, O. M. Alfano, and A. E. Cassano, Mass transfer limitations in slurry photocatalytic reactors: Experimental validation, Chemical Engineering Science 65, 4931 (2010).

[28] M. Motegh, J. Cen, P. W. Appel, J. R. van Ommen, and M. T. Kreutzer, 
Photocatalytic-reactor efficiencies and simplified expressions to assess their relevance in kinetic experiments, Chemical Engineering Journal 208, 607 (2012).

[29] Y. Matsushita, M. Iwasawa, T. Suzuki, and T. Ichimura, Multiphase Photocatalytic Oxidation in a Microreactor, Chemistry Letters 38, 846 (2009).

[30] X. Zhang, Photocatalytic microreactors for water purification: Selective control of oxidation pathways, The 8th Annual IEEE International Conference on Nano/Micro Engineered and Molecular Systems 1, 368 (2013).

[31] D. A. G. Bruggeman, Berechnung verschiedener physikalischer konstanten von heterogenen substanzen. $i$. dielektrizitatskonstanten und leitfahigkeiten der mischkarper aus isotropen substanzen, Annalen der Physik 416, 636 (1935).

[32] B. Johs and J. S. Hale, Dielectric Function representation by B-splines, Physica Status Solidi A: Applications and Materials Science 205, 715 (2008).

[33] M. G. Nielsen, S.-I. In, P. C. Vesborg, T. Pedersen, K. P. Almtoft, I. H. Andersen, O. Hansen, and I. Chorkendorff, A generic model for photocatalytic activity as a function of catalyst thickness, Journal of Catalysis $\mathbf{2 8 9}$, $62(2012)$.

[34] A. Einstein, A correction on my work-a new determination of molecular dimensions, (1969).

[35] M. Mooney, The viscosity of a concentrated suspension of spherical particles, Journal of Colloid Science 6, 162 (1951).

[36] I. M. Krieger, A Mechanism for Non-Newtonian Flow in Suspensions of Rigid Spheres, Journal of Rheology 3, 137 (1959).

[37] W. J. Tseng and K.-C. Lin, Rheology and colloidal structure of aqueous $\mathrm{TiO}_{2}$ nanoparticle suspensions, Materials Science and Engineering: A 355, 186 (2003).

[38] L. Lei, N. Wang, X. M. Zhang, Q. Tai, D. P. Tsai, and H. L. W. Chan, Optofluidic planar reactors for photocatalytic water treatment using solar energy, Biomicrofluidics 4, 31 (2010).

[39] Z. Meng, X. Zhang, and J. Qin, A high efficiency microfluidic-based photocatalytic microreactor using electrospun nanofibrous $\mathrm{TiO}_{2}$ as a photocatalyst, Nanoscale 5, 4687 (2013). 
[40] J. Kasanen, J. Salstela, M. Suvanto, and T. T. Pakkanen, Photocatalytic degradation of methylene blue in water solution by multilayer $\mathrm{TiO}_{2}$ coating on hdpe, Applied Surface Science 258, 1738 (2011).

[41] B. I. Stefanov, N. V. Kaneva, G. L. Puma, and C. D. Dushkin, Novel integrated reactor for evaluation of activity of supported photocatalytic thin films: Case of methylene blue degradation on $\mathrm{TiO}_{2}$ and nickel modified $\mathrm{TiO}_{2}$ under uv and visible light, Colloids and Surfaces A: Physicochemical and Engineering Aspects 382, 219 (2011).

[42] S. Zhang, Z. Chen, Y. Li, Q. Wang, and L. Wan, Photocatalytic degradation of methylene blue in a sparged tube reactor with $\mathrm{TiO}_{2}$ fibers prepared by a properly two-step method, Catalysis Communications 9, 1178 (2008).

[43] H. Xu, M. Li, and Z. Jun, Preparation, characterization, and photocatalytic studies on anatase nano- $\mathrm{TiO}_{2}$ at internal air lift circulating photocatalytic reactor, Materials Research Bulletin 48, 3144 (2013).

[44] Y. Wang, J. Lin, R. Zong, J. He, and Y. Zhu, Enhanced photoelectric catalytic degradation of methylene blue via $\mathrm{TiO}_{2}$ nanotube arrays hybridized with graphite-like carbon, Journal of Molecular Catalysis A: Chemical 349, 13 (2011).

[45] L. Rizzo, J. Koch, V. Belgiorno, and M. Anderson, Removal of methylene blue in a photocatalytic reactor using polymethylmethacrylate supported $\mathrm{TiO}_{2}$ nanofilm, Desalination 211, 1 (2007).

[46] H. Lachheb, E. Puzenat, A. Houas, M. Ksibi, E. Elaloui, C. Guillard, and J.M. Herrmann, Photocatalytic degradation of various types of dyes (alizarin $s$, crocein orange $g$, methyl red, congo red, methylene blue) in water by uvirradiated titania, Applied Catalysis B: Environmental 39, 75 (2002).

[47] A. Khataee, M. Fathinia, and S. Joo, Simultaneous monitoring of photocatalysis of three pharmaceuticals by immobilized $\mathrm{TiO}_{2}$ nanoparticles: Chemometric assessment, intermediates identification and ecotoxicological evaluation, Spectrochimica Acta Part A: Molecular and Biomolecular Spectroscopy 112, 33 (2013).

[48] K.-J. Hwang, J.-W. Lee, W.-G. Shim, H. D. Jang, S.-I. Lee, and S.-J. Yoo, Adsorption and photocatalysis of nanocrystalline $\mathrm{TiO}_{2}$ particles prepared by sol-gel method for methylene blue degradation, Advanced Powder Technology 
23, 414 (2012).

[49] J. Choi, H. Lee, Y. Choi, S. Kim, S. Lee, S. Lee, W. Choi, and J. Lee, Heterogeneous photocatalytic treatment of pharmaceutical micropollutants: Effects of wastewater effluent matrix and catalyst modifications, Applied Catalysis B: Environmental 147, 8 (2014).

[50] H. C. Aran, J. K. Chinthaginjala, R. Groote, T. Roelofs, L. Lefferts, M. Wessling, and R. G. H. Lammertink, Porous ceramic mesoreactors: A new approach for gas-liquid contacting in multiphase microreaction technology, Chemical Engineering Journal 169, 239 (2011).

[51] J.-M. Herrmann, Photocatalysis fundamentals revisited to avoid several misconceptions, Applied Catalysis B: Environmental 99, 461 (2010). 


\section{CHAPTER 6}

\section{Hydrogenation of nitrite in a membrane}

\section{microreactor}

The selectivity towards nitrogen is highly desirable in catalytic hydrogenation of nitrite. The selectivity is significantly influenced by the $H / N$ ratio of the reaction intermediates at the catalyst surface $(\mathrm{Pd})$. Here, we report on the fabrication and operation of a membrane reactor by which we separately feed hydrogen gas and nitrite solution. This arrangement realizes a homogeneous and tunable hydrogen concentration along the membrane reactor and consequently, low $\mathbf{H} / \mathbf{N}$ ratios and high selectivity towards nitrogen were obtained. The concept has been further investigated by comparing the performance of the membrane reactor with conventional reactor configurations where the hydrogen is dissolved in the nitrite solution.

This chapter is based on: Roger Brunet Espinosa, Damon Rafieian, Rob G.H. Lammertink, Leon Lefferts, Carbon nano-fiber based membrane reactor for selective nitrite hydrogenation, submitted to Catalysis Today. 


\subsection{Introduction}

Utilization of microreactors in multiphase reactions has received a great deal of attention. Due to at least one small dimension (characteristic length) they have excellent heat and mass transfer which is highly beneficial for chemical conversions. Moreover thanks to their small volume the process safety increases and residence time and heat can be accurately controlled [1]. The surface to volume ratio is remarkably high which makes them an ideal platform for heterogeneous catalytic reactions.

Membrane reactors also have several advantages for heterogeneous catalytic processes. The interface between separated phases is well-defined while the membrane itself can act as a support for the catalyst [2]. Sylvian et al. [3] categorize catalytic membrane reactors according to the role of membrane as extractor, distributor and contactor. In extractors, membranes function as separator of the products which have detrimental effect on the kinetics, from the reaction zone. As distributor the membrane is distributing at least one of the reactants homogeneously near the catalytic region. Finally in contactors, the membrane provides an optimum contact between the reactants and catalyst as a catalyst support.

The presence of nitrate and/or nitrite ions in drinking water is a growing public health concern worldwide. Although nitrate ions $\left(\mathrm{NO}_{3}{ }^{-}\right)$do not cause any toxicity its reduction to nitrite $\left(\mathrm{NO}_{2}{ }^{-}\right)$ions has adverse biological effect. Nitrite $\left(\mathrm{NO}_{2}{ }^{-}\right)$ions oxidize oxygen carrier molecule in blood, the hemoglobin, to methemoglobin rendering it unable to transport oxygen to the tissues. When the concentration of methemoglobin exceeds $10 \%$ of the normal hemoglobin molecules the condition is called methemoglobinemia (blue baby syndrome) [4]. For this reason the European Environmental Agency (EEA) has established a legal limit of 50 and $0.5 \mathrm{mg} / \mathrm{L}$ for nitrate and nitrite respectively $[5,6]$. The main sources of nitrate and/or nitrite groundwater pollution are the use of synthetic fertilizers and disposal of municipal effluents by sludge which is spread on the fields [7]. The conventional methods for nitrate or/and nitrite removal such as ion exchange, reverse osmosis and electrodialysis are not desirable due to the high cost of operations and the problem associated with post disposal of nitrate brine [8]. Moreover in all of these methods the ions become isolated instead of being de- 
composed to non-harmful products. On the other hand the elimination of the ions by biological degradation through microorganisms is not always favorable due to the concerns for pathogens and long process time [5].

Catalytic de-nitrification in water through hydrogenation of the nitrite and/or nitrate over noble-metal solid catalysts is proven to be an efficient method compared to conventional methods [6]. In this method firstly nitrate reduces to nitrite over a bimetallic catalyst (equation 6.1 ). This is followed by nitrite reduction to ammonia and nitrogen in the subsequent reactions (equation 6.2 and 6.3). The challenge of this method is the formation of ammonia which has a legal maximum contaminant level (MCL) of $0.5 \mathrm{mg} / \mathrm{L}$ as set by the EEA [5, 6]. Hence high selectivity to nitrogen is of paramount importance. The selectivity towards nitrogen is affected by many factors including $\mathrm{pH}$, temperature and hydrogen/nitrogen ratio $[9]$.

$$
\begin{aligned}
& 2 \mathrm{NO}_{3}^{-}+2 \mathrm{H}_{2} \stackrel{P d-C u}{\longrightarrow} 2 \mathrm{NO}_{2}^{-}+2 \mathrm{H}_{2} \mathrm{O} \\
& 2 \mathrm{NO}_{2}{ }^{-}+3 \mathrm{H}_{2} \stackrel{P d}{\longrightarrow} \mathrm{N}_{2}+4 \mathrm{H}_{2} \mathrm{O} \\
& \mathrm{NO}_{2}^{-}+3 \mathrm{H}_{2}+2 \mathrm{H}^{+} \stackrel{P d}{\longrightarrow} \mathrm{NH}_{4}^{+}+2 \mathrm{H}_{2} \mathrm{O}
\end{aligned}
$$

In this study, we investigate the use of a contactor and distributor membrane microreactor for the nitrite hydrogenation reaction. The contactor part consists of an alumina hollow fiber (AHF) structure impregnated with carbon nanofibers (CNFs) as catalyst support for palladium nanoparticles. A thin layer of polydimethylsiloxane (PDMS) functioning as distributor membrane covered the contactor part. As such, the hydrogen can be dosed in the reaction zone inside the contactor part. By hydrogen dilution with argon low $\mathrm{H} / \mathrm{N}$ ratios can be realized and by using the PDMS layer as the distributor membrane low and homogeneous hydrogen concentration is distributed along the catalyst bed. The reactor is illustrated in figure 6.1. Although membrane reactors have been studied for nitrate and nitrite hydrogenation [9-13], tuning of the selectivity via manipulation of the $\mathrm{H} / \mathrm{N}$ ratio has not yet been demonstrated. Operation at low $\mathrm{H} / \mathrm{N}$ ratio with nitrite solution pre-saturated with hydrogen would not be efficient, as exhaustion 
of dissolved hydrogen would cause very low nitrite conversion and mass transfer limitation. The membrane microreactor in this work (figure 6.1) functions (figure 6.1 ) at very low hydrogen concentration while preventing hydrogen diffusion limitation.

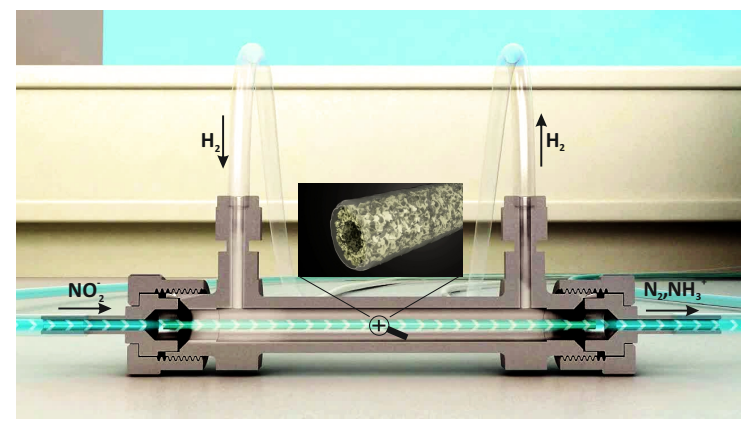

Figure 6.1: The alumina hollow fiber based microreactor concept. The coated PDMS layer on the outside of the alumina hollow fiber acts as a distributor membrane. This configuration provides low and homogeneous hydrogen concentration along the contactor membrane, preventing hydrogen depletion.

\subsection{Experimental}

The preparation of the membrane reactor includes multiple steps. Firstly, nickel was deposited in the macropores of the alumina hollow fiber functioning as the catalyst for the subsequent growth of CNF. Poorly attached CNFs were removed by sonication. Next, palladium as the hydrogenation catalyst was deposited on the CNFs followed by calcination and reduction. Finally, the outer wall of the alumina fiber was coated with a viscous PDMS solution, forming a dense layer after curing. Below, each step is explained separately in detail. The complete fabrication steps are schematically illustrated in figure 6.2.

Nickel deposition: An $\alpha$-alumina hollow fiber (Hyflux CEPAration Technologies, Europe) with inner and outer diameter of 0.9 and $1.9 \mathrm{~mm}$ respectively was cut in $55 \mathrm{~mm}$ long piece and without any treatment submerged inside a wellstirred Ni nitrate hexahydrate (Merck) solution $(80 \mathrm{~mL})$. Different concentrations were prepared including $0.02,0.2,1.0$ and $5.0 \mathrm{gNi} / \mathrm{L}$. The initial $\mathrm{pH}$ of the solution was adjusted to 3.5 using a diluted nitric acid (65\%, Merck) solution. The 
temperature was kept constant at $100{ }^{\circ} \mathrm{C}$ using a reflux system. To precipitate the nickel inside the alumina hollow fiber's pores, $20 \mathrm{ml}$ of concentrated urea solution (Merck) $(1.06 \mathrm{~g} / 20 \mathrm{ml})$ was added drop-wise for the first 15 minutes of the process. After this the deposition continues for 2 hours under the same conditions. Finally, the nickel deposited alumina hollow fiber was taken out from the nickel solution, rinsed thoroughly with miliQ water and dried at $85{ }^{\circ} \mathrm{C}$ for two hours (figure $6.2(\mathrm{a})$ ).

CNF growth: Catalytically controlled thermal chemical vapor deposition (C-TCVD) technique was employed for the growth of CNFs. The synthesis was carried out inside a quartz tube reactor with an inner diameter of $10 \mathrm{~mm}$. Initially the hollow fiber was reduced in $20 \% \mathrm{H}_{2}$ and $80 \% \mathrm{~N}_{2}$ mixture at different temperatures $\left(450,550,650,750\right.$ or $\left.850{ }^{\circ} \mathrm{C}\right)$ for 2 hours. The temperature was increased from $20{ }^{\circ} \mathrm{C}$ to the target temperature at $6{ }^{\circ} \mathrm{C} / \mathrm{min}$ under $100 \mathrm{ml} / \mathrm{min}$ $\mathrm{N}_{2}$. This is followed by the synthesis step which was carried out at $(350,450$, $550,600,700,750{ }^{\circ} \mathrm{C}$ ) in a gas mixture containing $7 \% \mathrm{H}_{2}, 20 \%$ ethylene $\mathrm{C}_{2} \mathrm{H}_{4}$ (99.95\% PRAXAIR) and $73 \% \mathrm{~N}_{2}$ with a total flow rate of $100 \mathrm{ml} / \mathrm{min}$. At the end, the system was cooled down to room temperature in a nitrogen atmosphere. In order to remove the poorly attached CNFs the sample was sonicated in milliQ water for 30 minutes. After this the alumina hollow fiber was dried at $85{ }^{\circ} \mathrm{C}$ for 2 hours. (figure $6.2(\mathrm{~b})$ )

Pd deposition: The alumina hollow fiber (AHF) was immersed in $25 \mathrm{ml}$ of palladium acetylacetonate (Alfa Aesar) and toluene (99.9\%, Merck) solution with concentration of $6 \mathrm{mg} \mathrm{Pd} / \mathrm{ml}$ for 17 hours. This is followed by drying at $85{ }^{\circ} \mathrm{C}$ for 2 hours. Finally the sample was calcined in air and reduced in a gas mixture of $50 \% \mathrm{H}_{2}$ and $50 \% \mathrm{~N}_{2}$ at $250{ }^{\circ} \mathrm{C}$ for 2 hours. (figure 6.2 (c))

PDMS coating: Since the viscosity of the pristine PDMS is too high for coating AHFs and very low viscosity results in a membrane infiltration, the diluted PDMS is pre cross-linked prior to coating. Adapted from [14], a two component PDMS kit (RTV-A and RTV-B, 10:1 weight ratio) was dissolved in toluene at $85 \mathrm{wt} \%$ and heated at $60{ }^{\circ} \mathrm{C}$ in a home-made reflux setup for pre-crosslinking the PDMS/toluene solution. The viscosity of the solution was measured by a Brookfield DV-II + Pro viscometer equipped with a nr-61 spindle. When 100 mPa.s was reached, the cross-linking was stopped by immersing the solution in 
ice. The surface tension of the solution was measured by Du Noüy ring method using a tensiometer (Krüss easy Dyne). The AHF was dip-coated with the partial cross-linked solution at a constant speed of $2.5 \mathrm{~mm} / \mathrm{s}$. To prevent the presence of PDMS inside the alumina fiber, one end of the alumina was sealed by glue and the open end was kept above the PDMS solution during the coating. In the last step, the coated sample was completely cross-linked in an oven at $80^{\circ} \mathrm{C}$ for 2 hours. Finally, both ends of the alumina were cut to remove the glue at one side and the non-coated part on the other side. (figure $6.2(\mathrm{~d})$ )

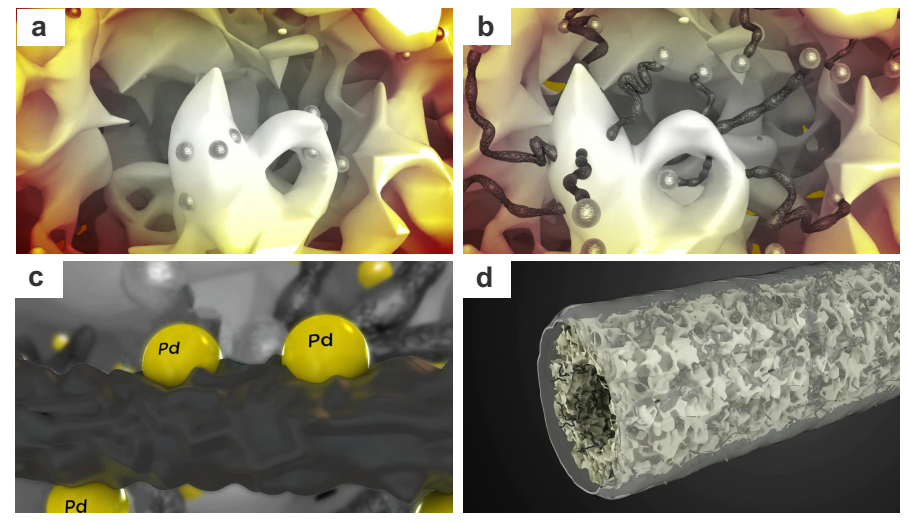

Figure 6.2: The fabrication steps of the membrane reactors. a) Deposition of the nickel as the catalyst for the growth of CNFs inside the pores of alumina hollow fiber. b) C-TCVD growth of carbon nanofibers. c) Deposition of Pd over the CNFs d) The PDMS layer coated over the alumina hollow fiber.

\subsection{Catalytic assessment}

The membrane reactor, from here on named " $\mathrm{H}_{2}$ outside", was tested for the nitrite hydrogenation to assess its performance in a fast liquid phase reaction. The performance of this reactor was compared with two other reactor configurations (" $\mathrm{H}_{2}$ inside" and "Packed bed"), as described in detail below. All reactions were carried out in liquid phase at $20^{\circ} \mathrm{C}$. Inlet and outlet concentrations of nitrite $\left(\mathrm{NO}_{2}{ }^{-}\right)$and ammonia $\left(\mathrm{NH}_{4}{ }^{+}\right)$were measured with an in-line Ion Chromatograph (Dionex, ICS 1000) from which $\mathrm{NO}_{2}{ }^{-}$conversion and ammonia selectivity were calculated according to equation 6.4 and 7.3 respectively. Assuming nitrogen and ammonia are the only products $[5,15,16]$, the nitrogen selectivity based on the 
mass balance (equation 6.2, 6.3) was calculated. The liquid flow rate was varied between 0.1 and $3 \mathrm{ml} / \mathrm{min}$ and the gas flow rate between 100 and $200 \mathrm{ml} / \mathrm{min}$ keeping the hydrogen partial pressure between 0.04 and 1.00 bar (balanced with Ar). The nitrite conversions were kept constant when selectivities are compared between the different reactor configuration. In all cases, the solutions were not buffered.

$$
\begin{aligned}
& \mathrm{NO}_{2}{ }^{-} \text {conversion }=\frac{\left[\mathrm{NO}_{2}{ }^{-}\right]_{\text {initial }}-\left[\mathrm{NO}_{2}{ }^{-}\right]_{\text {final }}}{\left[\mathrm{NO}_{2}{ }^{-}\right]_{\text {initial }}} .100 \\
& \mathrm{NH}_{4}{ }^{+} \text {selectivity }=\frac{\left[\mathrm{NH}_{4}{ }^{+}\right]_{\text {final }}-\left[\mathrm{NH}_{4}{ }^{+}\right]_{\text {initial }}}{\left[\mathrm{NO}_{2}{ }^{-}\right]_{\text {initial }}-\left[\mathrm{NO}_{2}{ }^{-}\right]_{\text {final }}} .100
\end{aligned}
$$

\subsection{1 $\mathrm{H}_{2}$ outside reactor}

This reactor configuration concerns a distributor (PDMS) and contactor (AHF) membrane. The distributor membrane (PDMS) provides low and homogeneous hydrogen concentrations along the axial direction of contactor membrane (AHF). The liquid containing nitrite is fed to the AHF (lumen) while the gas is fed to the shell of the reactor and is allowed to diffuse through the PDMS membrane which is coated on the outer surface of the AHF (figure 6.3). Hydrogen and nitrite are expected to react in the macropores of the $\alpha-\mathrm{Al}_{2} \mathrm{O}_{3}$ where palladium is deposited on the entangled CNFs (figure $6.2(\mathrm{c})$ ). In all experiments, liquid is saturated in argon to remove oxygen and other gases present in ambient which could interfere with the hydrogenation reaction [17]. The liquid flow rate was fixed at $0.2 \mathrm{ml} / \mathrm{min}$ with a nitrite concentration between 20 and $1000 \mathrm{~mol} / \mathrm{L}$. The gas flow rate was supplied at 1 bar, feeding a mixture of hydrogen and argon with the hydrogen partial pressure varied between 0.05 and 1 bar.

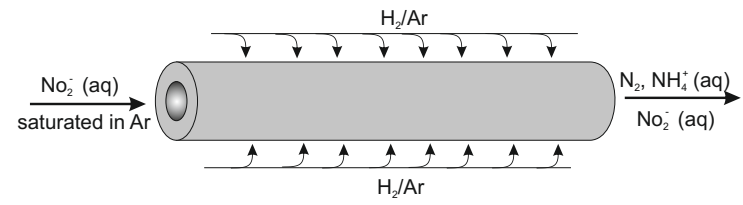

Figure 6.3: " $\mathrm{H}_{2}$ outside" reactor configuration 


\subsection{2 $\mathrm{H}_{2}$ inside reactor}

The reactor was physically similar to "H2 outside" but it was operated differently. In this design, the liquid containing nitrite was pre-saturated with hydrogen at 1 bar and fed inside the tube side of the AHF reactor. Pure argon was supplied at 1 bar to the reactor shell to avoid any permeation of air to the reaction zone (figure 6.4 ). The liquid flow rate was $0.2 \mathrm{ml} / \mathrm{min}$ with a nitrite and hydrogen concentrations of $220 \mu \mathrm{mol} / \mathrm{L}$ and $780 \mu \mathrm{mol} / \mathrm{L}$ respectively. Hydrogen concentration in the liquid feed was controlled by the pressure of the hydrogen used to saturate the nitrite solution and was calculated based on Henry's law constant $\left(7.7\right.$ mol.m $\left.{ }^{-3} \cdot \mathrm{Pa}^{-1},[18]\right)$.

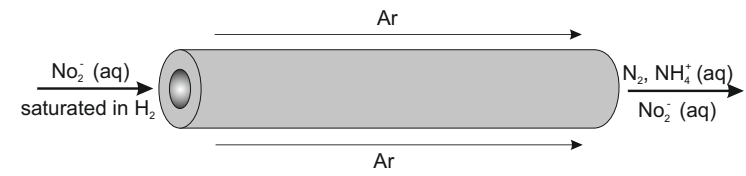

Figure 6.4: " $\mathrm{H}_{2}$ inside" reactor configuration

\subsubsection{Packed bed reactor}

A membrane reactor without any PDMS coating was crushed and sieved to the size of $0.5 \mathrm{~mm}$ particles and was tested as a packed bed reactor. The reactor was fed with a nitrite solution pre-saturated in hydrogen at 1 bar (figure 6.5). The liquid flow rate was $3 \mathrm{ml} / \mathrm{min}$ to obtain the same conversion compared to the other reactor layouts. Additionally, one experiment was performed with a low flow rate $(0.4 \mathrm{ml} / \mathrm{min})$. Nitrite and hydrogen inlet concentrations were kept at $220 \mu \mathrm{mol} / \mathrm{L}$ and $780 \mu \mathrm{mol} / \mathrm{L}$ respectively.

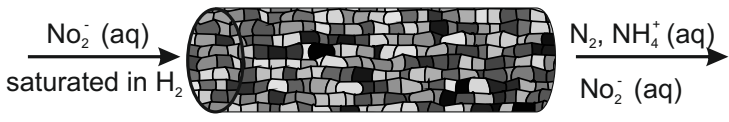

Figure 6.5: "packed bed" reactor configuration 


\subsection{Characterization}

The BET surface area of the AHFs after CNF growth was obtained from $\mathrm{N}_{2}$ adsorption isotherm obtained at $77 \mathrm{~K}$ (Micromeritics Tristar). The morphology of membrane reactors was studied with Scanning Electron Microscopy, HR-SEM (Analysis Zeiss MERLIN HR-SEM). The studied properties include the CNF diameter, nickel particle size and thickness of the PDMS layer. CNF diameter and nickel particle size (before CNF growth) were determined based on the average of 200 measurements on five different positions in the sample. The amount of carbon grown in the macropores of the $\alpha$-alumina was measured with an analytical balance by weighing the sample before and after CNF growth. Moreover, elemental analysis, EA (Elemental Analyser Inter Science Flash 2000) was utilized to determine the amount of carbon deposited in the AHF. The average pore size and the void fraction of the $\alpha$-alumina before the CNF growth were determined with Hg porosimetry (Quantachrome Poremaster PM33). The percentage of pore volume filled with CNFs was calculated from the void fraction of the $\alpha$-alumina and the amount of CNFs. The Pd loading on the membrane reactor was determined with X-ray fluorescence spectroscopy, XRF (Philips PW 1480).

\subsection{Results and Discussion}

Figure 6.6 presents the general cross-sectional view and high magnification of the pristine $\alpha$-alumina hollow fiber. The pore sizes are $\sim 800 \mathrm{~nm}$ in agreement with the manufacturer's specification. The porosity and BET surface area is 0.13 $\mathrm{cm}^{3} / \mathrm{g}$ and $\sim 0.1 \mathrm{~m}^{2} / \mathrm{g}$ respectively.

\subsubsection{Nickel deposition}

Figure 6.7 shows the morphology of the CNFs inside the macropores of alumina hollow fiber at different concentrations of nickel precursor. All of the CNFs growth were performed at $450{ }^{\circ} \mathrm{C}$ pretreatment in hydrogen and synthesis at $450{ }^{\circ} \mathrm{C}$. At low nickel concentration $(0.02 \mathrm{gNi} / \mathrm{L})$ there are few CNFs covering the alumina walls as shown in figure 6.7(a). This is further confirmed by low carbon content $(0.2 \mathrm{wt} \%)$ leading to a very poor filling of the alumina macropores 


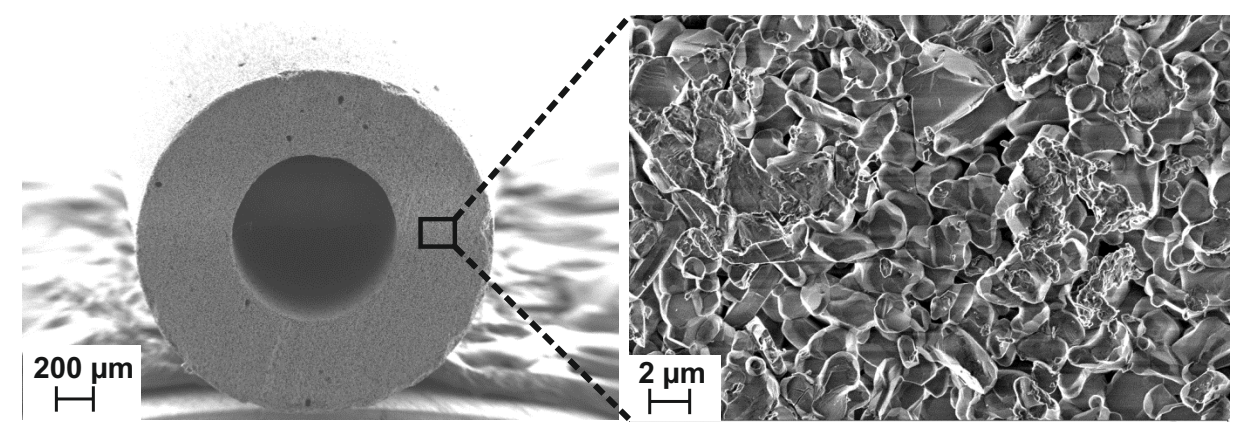

Figure 6.6: General cross sectional view (left) and high magnification (right) of a pristine $\alpha$-alumina hollow fiber

(approx. 0.6 vol\%) and very low surface area of $\sim 0.4-0.5 \mathrm{~m}^{2} / \mathrm{g}$. At high nickel concentration a thick layer $(200 \mu \mathrm{m})$ of carbon nanofibers has grown on the outer surface of the alumina fiber (figure 6.7(d)). This massive CNF growth has detrimental effect on the mechanical stability of the AHF [19].

The samples prepared with $0.2 \mathrm{gNi} / \mathrm{L}$ and $1 \mathrm{gNi} / \mathrm{L}$ show a favorable CNF coverage (figure $6.7(\mathrm{~b}, \mathrm{c})$ ). Among these two, the sample prepared at $1 \mathrm{gNi} / \mathrm{L}$ was selected for the rest of the process which possesses the highest carbon content and as a result the highest surface area $\left(\sim 10 \mathrm{~m}^{2} / \mathrm{g}\right)$.

\subsubsection{Reduction temperature}

In case of high reduction temperatures $\left(850{ }^{\circ} \mathrm{C}\right.$ and $\left.750{ }^{\circ} \mathrm{C}\right)$, CNFs present slightly larger diameter than at low reduction temperatures due to the sintering of the nickel particles and exhibit smaller, but still significant, BET surface areas $\left(5.6 \mathrm{~m}^{2} / \mathrm{g}\right)$ than at $550{ }^{\circ} \mathrm{C}\left(10.2 \mathrm{~m}^{2} / \mathrm{g}\right)$. However, high reduction temperatures are preferred since CNFs then offer an open structure, reducing the risk of mass transfer limitations. Among the two high reduction temperatures, $850{ }^{\circ} \mathrm{C}$ (figure 6.8 (a)) was selected as the reduction temperature for further optimization. The diameter of the CNFs corresponds to the nickel particle size, which in turn can be manipulated during the reduction process. Here we investigate the effect of temperature on the nickel particle size and CNFs accordingly. Four different temperatures were tested followed by $\mathrm{CNF}$ synthesis at $450{ }^{\circ} \mathrm{C}$. When low reduction temperatures $\left(550{ }^{\circ} \mathrm{C}\right.$ and $\left.450{ }^{\circ} \mathrm{C}\right)$ are applied, straight and very thin 

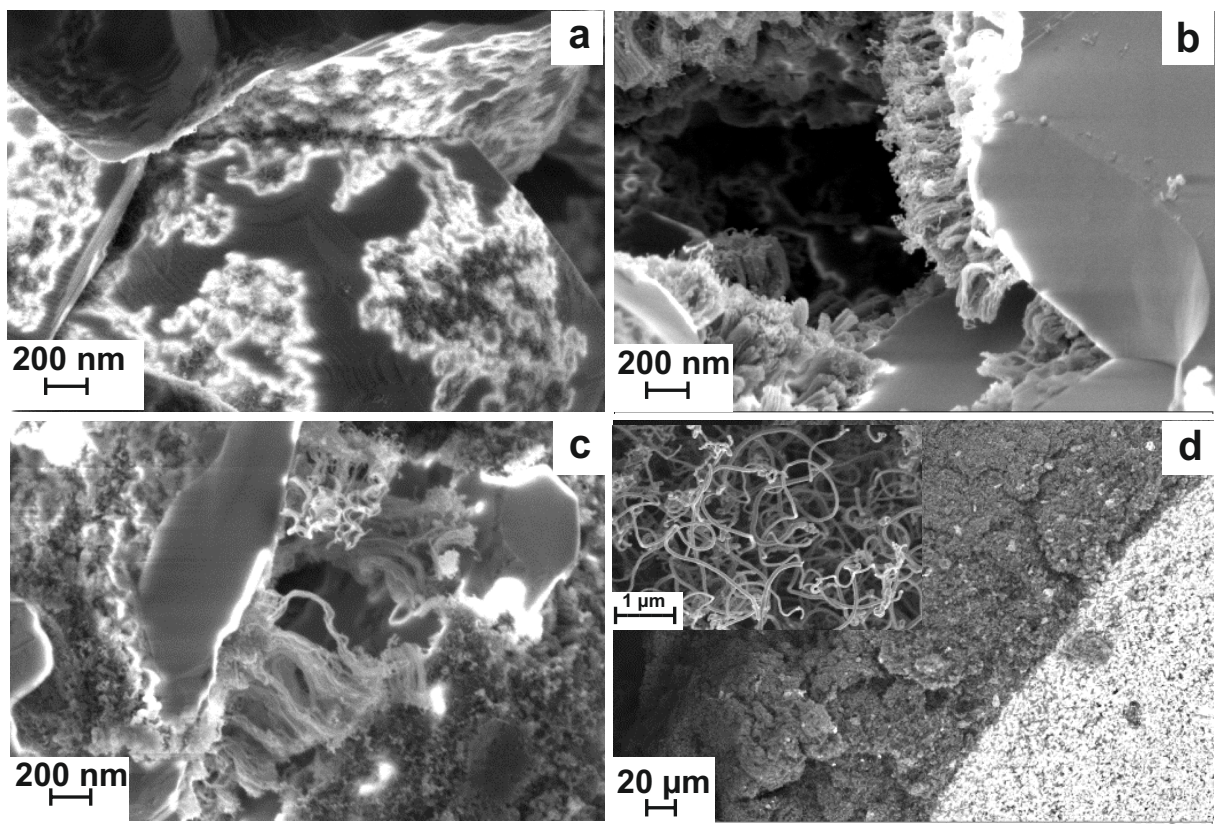

Figure 6.7: SEM micrographs of alumina macropores with grown CNFs using a) 0.02 $\mathrm{gNi} / \mathrm{L}, \mathrm{b}) 0.2 \mathrm{gNi} / \mathrm{L}$, c) $1 \mathrm{gNi} / \mathrm{L}$ and d) $5.0 \mathrm{gNi} / \mathrm{L}$ nickel precursor concentrations. Higher magnification is shown in the insert.

CNFs grow parallel next to each other, generating groups of packed CNFs that resemble amorphous carbon (figure 6.8 (c) and (d)). This morphology, with very small pores between the packed CNFs, would not be beneficial as the catalyst support.

\subsubsection{CNF growth temperature}

The CNFs were grown using the above mentioned optimized conditions for nickel deposition and reduction temperature but at different growth temperatures ranging from $300{ }^{\circ} \mathrm{C}$ to $750{ }^{\circ} \mathrm{C}$.

As observed in figure 6.9 (b-e) CNFs are present in all of the samples grown at the temperature between $450{ }^{\circ} \mathrm{C}$ and $700{ }^{\circ} \mathrm{C}$. CNFs grown at $550{ }^{\circ} \mathrm{C}$ present the highest surface area $\left(21.0 \mathrm{~m}^{2} / \mathrm{g}\right)$ and relatively low pore filling (17.2 vol\%). CNFs are not well-defined and in some areas densely packed CNFs resemble the morphology of amorphous carbon as seen in figure $6.9(\mathrm{~g})$. The highest CNF 

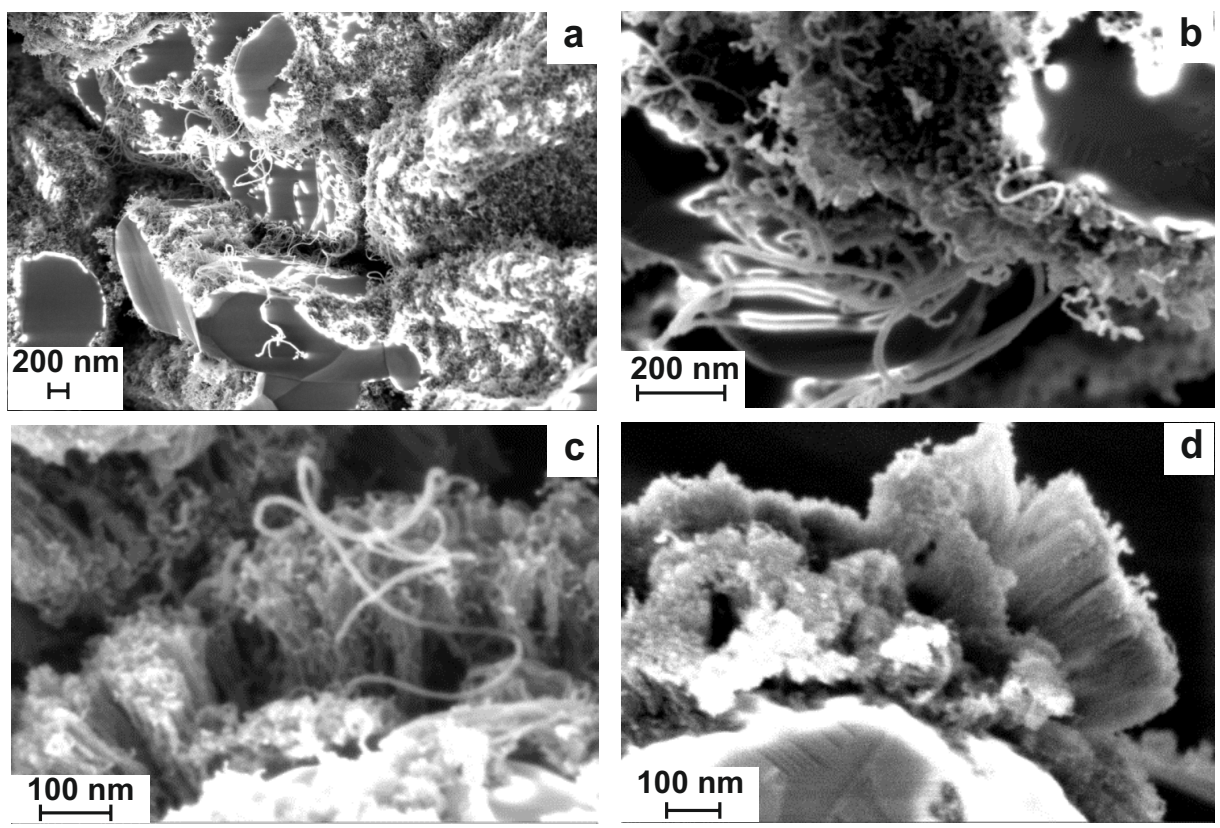

Figure 6.8: SEM micrographs of the grown CNFs synthesized at $450{ }^{\circ} \mathrm{C}$ and reduced at a) $850{ }^{\circ} \mathrm{C}$, b) $750{ }^{\circ} \mathrm{C}$, c) $550{ }^{\circ} \mathrm{C}$ and d) $450{ }^{\circ} \mathrm{C}$.

amount $(7.5 \mathrm{wt} \%)$ and relatively high surface area $\left(16.8 \mathrm{~m}^{2} / \mathrm{g}\right)$ belongs to the sample grown at $600{ }^{\circ} \mathrm{C}$. In addition CNFs grown at $600{ }^{\circ} \mathrm{C}$ are homogeneously distributed and do not agglomerate forming packed areas (figure $6.9(\mathrm{~h})$ ).

Table 6.1: Synthesis parameters and properties of the optimized sample.

\begin{tabular}{ll}
\hline \multicolumn{2}{c}{ Optimized sample } \\
\hline Nickel concentration $(\mathrm{gNi} / \mathrm{L})$ & 1.0 \\
Reduction temperature $\left({ }^{\circ} \mathrm{C}\right)$ & 850 \\
Growth temperature $\left({ }^{\circ} \mathrm{C}\right)$ & 600 \\
wt\% C & 7.5 \\
Surface area $\left(\mathrm{m}^{2} / \mathrm{g}\right)$ & 16.8 \\
Pore volume filled $(\mathrm{Vol} \%)$ & 25 \\
Palladium loading $(\mathrm{gPd} / \mathrm{gCNF})$ & 0.011 \\
\hline
\end{tabular}

The growth of CNFs at $350{ }^{\circ} \mathrm{C}$ is hindered because this temperature is too low to allow the carbon containing gas diffuses through and around the nickel particles which is indispensable for the growth of CNFs. (figure 6.9 (a)) [20]. At 
the highest $\mathrm{CNF}$ growth temperature $\left(750{ }^{\circ} \mathrm{C}\right)$, the $\mathrm{CNF}$ coverage was extremely low (figure 6.9 (f)), showing only few and dispersed long CNFs at the internal surface of the alumina hollow fiber.

The synthesis parameters and the properties of the optimized sample in terms of nickel deposition, reduction and CNFs' growth temperature are summarized in table 6.1 . 

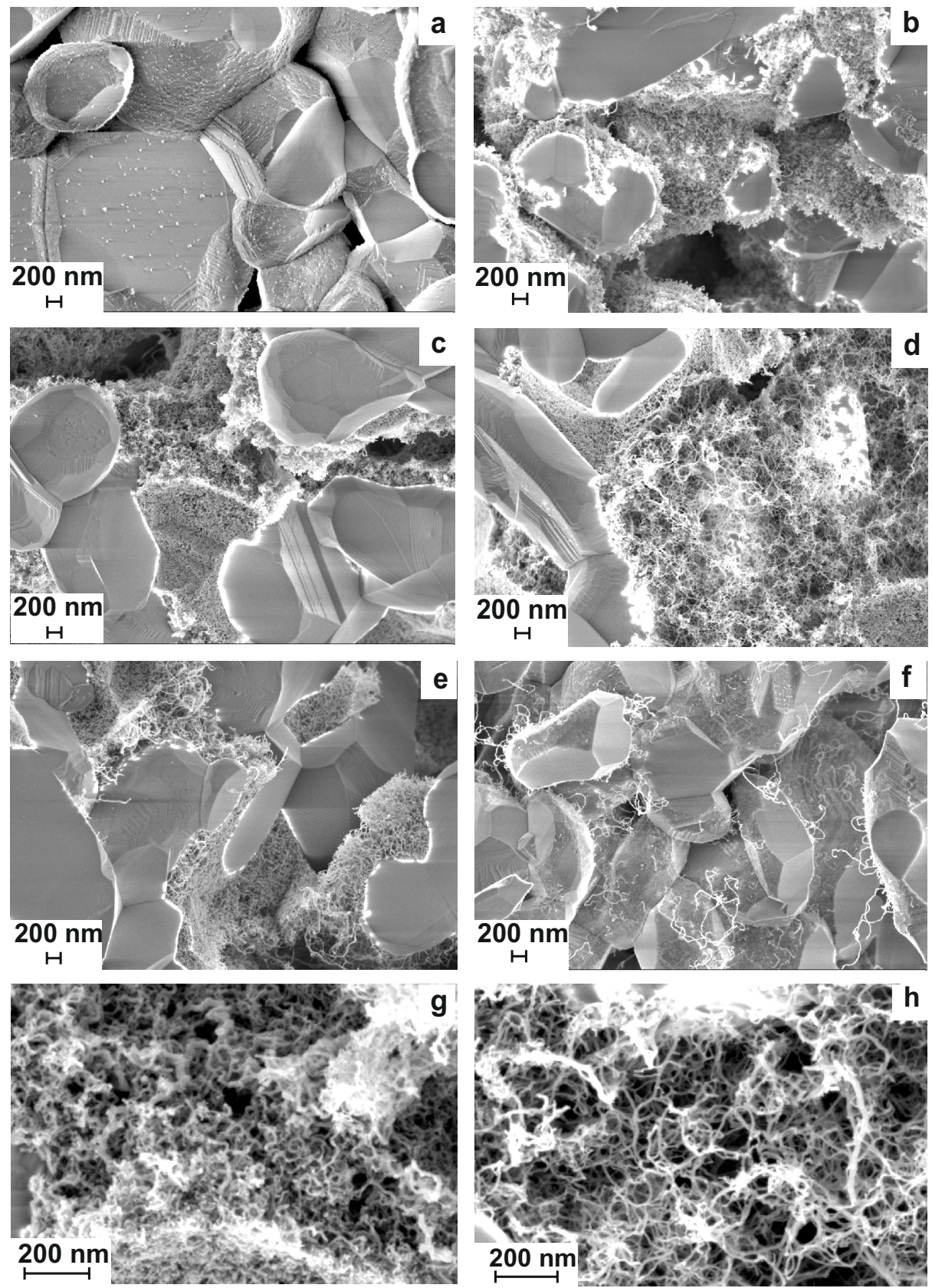

Figure 6.9: SEM micrographs of the grown CNFs synthesized at a) $350{ }^{\circ} \mathrm{C}$, b) $450{ }^{\circ} \mathrm{C}$, c) $550{ }^{\circ} \mathrm{C}$, d) $600{ }^{\circ} \mathrm{C}$, e) $700{ }^{\circ} \mathrm{C}$, f) $750{ }^{\circ} \mathrm{C}$, g) $550{ }^{\circ} \mathrm{C}$ (high magnification) and h) 600 ${ }^{\circ} \mathrm{C}$ (high magnification) 


\subsubsection{PDMS coating}

Figure 6.10 depicts the change in the viscosity of PDMS/toluene solution as a function of crosslinking time. The viscosity increases abruptly reaching to 100 mPa.s within only 30 minutes between 270 and 300 minutes. At this viscosity a thin coating layer $(\sim 10 \mu \mathrm{m})$ which is homogeneous in both axial and radial direction can be realized (figure 6.10 (insert)). The PDMS coating remained intact after even 400 hours time-on-stream which confirms its mechanical stability. The thickness of the coating outside was predicated by Bretherton's law [21] (equation 6.6) where $b$ is the outer diameter of the fiber and Ca is capillary number. Capillary number is defined by $\mathrm{Ca}=\eta \mathrm{V} / \gamma$ where $\eta, \mathrm{V}$ and $\gamma$ are viscosity, average velocity of dip coating and surface tension of the solution, respectively. The predicted value is $\sim 40 \mu \mathrm{m}$ which is larger than the obtained thickness. The observed discrepancy could be attributed to the infiltration of diluted PDMS into the porous structure of the AHF. Another possible explanation may be the inaccuracies in viscosity and surface tension measurements.

$$
\text { Coating Thickness }=b C a^{2 / 3}
$$

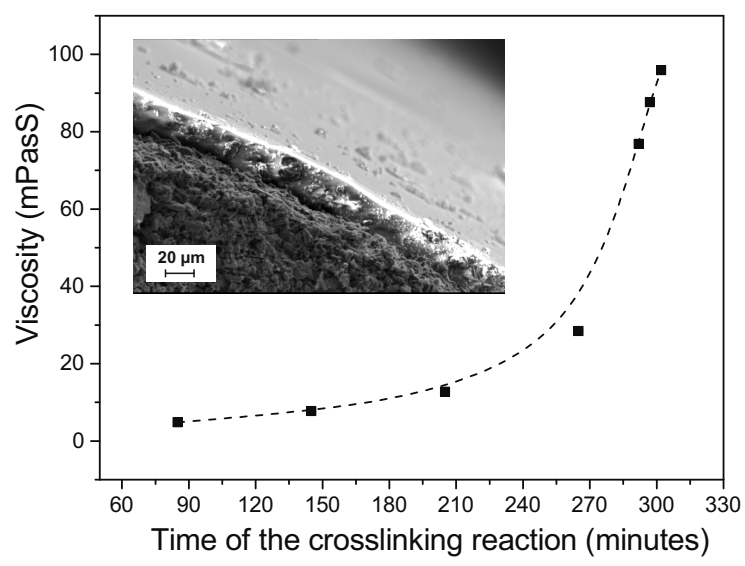

Figure 6.10: Change of the viscosity of a $85 \%(\mathrm{w} / \mathrm{w})$ PDMS coating solution versus crosslinking time. The insert shows a hollow alumina fiber with CNFs grown in the macropores and a $\sim 10 \mu \mathrm{m}$ thick PDMS layer coated on its outer surface. 


\subsubsection{Catalytic nitrite hydrogenation}

\section{Effect of reactor design on ammonia selectivity}

The three reactor configurations explained in the experimental section (" $\mathrm{H}_{2}$ outside", " $\mathrm{H}_{2}$ inside" and "Packed bed") were assessed for nitrite hydrogenation. The synthesis parameters and properties of the all three reactors are indicated in table 6.1. As observed in figure 6.11 " $\mathrm{H}_{2}$ outside" is less selective towards ammonia compared to the other reactors. The hydrogen partial pressure in the reactor shell is 0.2 bar, corresponding to $156 \mu \mathrm{mol} \mathrm{H}_{2} / \mathrm{L}$ in the liquid according to hydrogen solubility. It should be noted that at the reaction zone the $\mathrm{H}_{2}$ concentration is possibly lower due to hydrogen transport resistance exerted by the PDMS coating. The role of the PDMS coating is more pronounced when the reactor is compared with a similar reactor concept used by Aran et al. [10]. They used an alumina hollow fiber with an internal hydrophilic $\gamma-\mathrm{Al}_{2} \mathrm{O}_{3}$ layer in contact with nitrite solution and an external hydrophobic $\alpha$-alumina layer in contact with the gas. Although they modified the hydrogen concentration from $10 \%$ to $100 \%$, they did not observe any remarkable influence on the ammonia selectivity. This is due to the almost inexistent diffusion resistance at the gas-liquid interface at the non-wetting hydrophobic $\alpha$-alumina macropores. The high diffusion coefficient of hydrogen in gas phase (typically $1 \mathrm{~cm}^{2} / \mathrm{s}$ ) [22] allows easy access of hydrogen to the reaction zone for all tested conditions. In the " $\mathrm{H}_{2}$ inside" and the "Packed bed" reactors, which are two phase systems, the concentration of hydrogen is $780 \mu \mathrm{mol} \mathrm{H} \mathrm{H}_{2} / \mathrm{L}$ which makes the $\mathrm{H}_{2} / \mathrm{N}_{2}$ ratio more than five times higher than the " $\mathrm{H}_{2}$ outside" reactor. Low $\mathrm{H}_{2} / \mathrm{N}_{2}$ ratios with " $\mathrm{H}_{2}$ inside" and the "Packed bed" reactors can not be realized due to hydrogen depletion (exhaustion). For instance operation at $156 \mu \mathrm{mol} \mathrm{H}_{2} / \mathrm{L}$, would lead to a maximum nitrite conversion of only $30 \%$.

Figure 6.12 shows that the ammonia selectivity increases by increasing $\mathrm{H}_{2}$ concentration in the " $\mathrm{H}_{2}$ outside" reactor. In addition, by increasing nitrite concentration the selectivity towards ammonia decreases figure 6.13. This stresses the significant role of the $\mathrm{H}_{2} / \mathrm{N}_{2}$ ratio in the reaction selectivity which has been also reported in literature $[6,16,23,24]$. The advantage of this reactor configuration is the possibility to manipulate this ratio and as a result tune the 


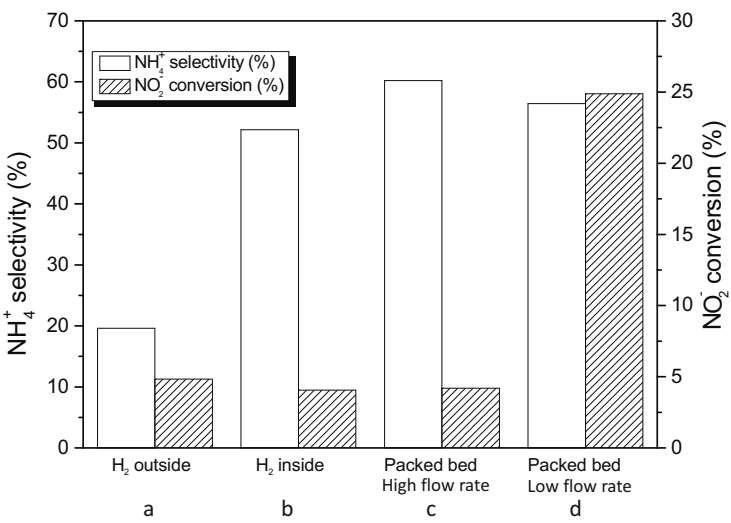

Figure 6.11: a) $\mathrm{H}_{2}$ pressure 0.2 bar, liquid flow rate $0.2 \mathrm{ml} / \mathrm{min}$, b) $\mathrm{H}_{2}$ pressure 0.2 bar, liquid flow rate $0.2 \mathrm{ml} / \mathrm{min} \mathrm{c}$ ) $\mathrm{H}_{2}$ concentration $780 \mathrm{~mol} \mathrm{H}_{2} / \mathrm{L}$ (pre-saturated at 1bar), liquid flow rate $3.0 \mathrm{ml} / \mathrm{min}$, d) $\mathrm{H}_{2}$ concentration $780 \mathrm{~mol} \mathrm{H}_{2} / \mathrm{L}$ (pre-saturated at 1 bar), liquid flow rate $0.4 \mathrm{ml} / \mathrm{min}$.

selectivity to nitrogen. Despite the noticeable change in selectivity by altering the $\mathrm{H}_{2}$ concentration the conversion remained almost constant. The minor decrease in conversion at very low hydrogen concentration suggests a low reaction order for hydrogen. This is in agreement with reaction orders reported in literature for hydrogenation reactions with $\mathrm{Pd}-\mathrm{Cu} / \mathrm{C}$ [25] and $\mathrm{Pd}-\mathrm{CNF}-\mathrm{Ni}$ foam [16]. Even zero order was reported for Pd on carbon cloth [26]. The low reaction order of 0.4 was also found for nitrite.

Low reaction order for both reactants and the strong effect of their concentration on the selectivity suggest a Langmuir-Hinshelwood mechanism with competitive adsorption of hydrogen and nitrite with intermediate surface coverages of both reactants. Low reaction orders are caused by the fact that the rate is related to the product of nitrite and hydrogen coverages $\left(\theta_{\mathrm{NO}_{2}}{ }^{*} \theta_{\mathrm{H}_{2}}\right)$. A coverage decrease of one of the reactants is compensated with a coverage increase of the other reactant, having a minor effect on the reaction rate but a significant effect on the selectivity because of the variation in the $\theta_{\mathrm{NO}_{2}}{ }^{-} / \theta_{\mathrm{H}_{2}}$ ratio. A Langmuir-Hinshelwood mechanism with different adsorption sites for nitrite and hydrogen can be rejected because it cannot explain the observation that the hydrogen concentration has a minor effect on the reaction rate and a strong effect on the selectivity.

" $\mathrm{H}_{2}$ outside" layout exhibits the same reaction rate compared to " $\mathrm{H}_{2}$ inside" 


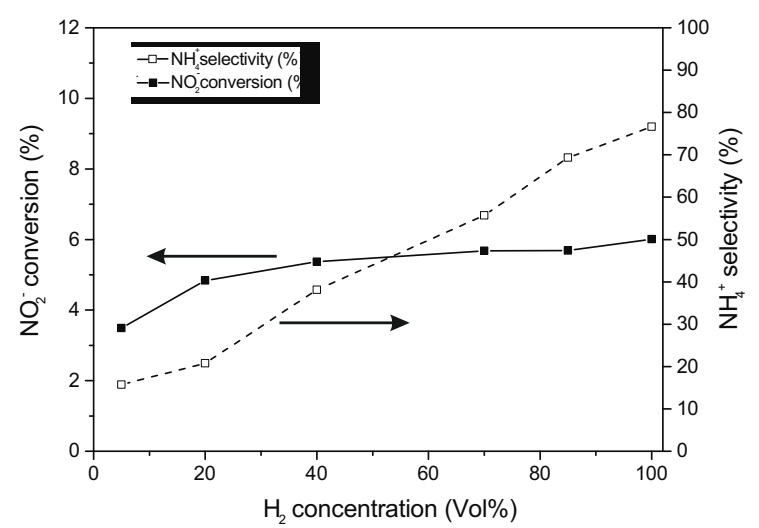

Figure 6.12: Nitrite conversion and ammonia selectivity of the " $\mathrm{H}_{2}$ outside" layout at different $\mathrm{H}_{2}$ concentrations. WHSV $\left(0.35 \mathrm{~h}^{-1}\right)$ and nitrite concentration $(220 \mu \mathrm{mol} / \mathrm{L})$ were kept constant.

but much lower than "Packed bed". Table 6.2 shows that the "Packed bed" layout has a much higher weight hourly space velocity (WHSV) than " $\mathrm{H}_{2}$ outside" and " $\mathrm{H}_{2}$ inside" when all the layouts present the same conversion (4-5\%). The two experiments performed with the Packed bed layout showed that at low liquid flow rate (high conversion) the reaction rate is lower than at high liquid flow rate (low conversion). This is probably caused by a deviation from differential conditions, where at higher conversion, averaged nitrite and hydrogen concentrations decrease, generating lower rates.

The difference in reaction rate between Packed bed and the other layouts could be caused by a significant by-passing of the reactants in the " $\mathrm{H}_{2}$ inside" and " $\mathrm{H}_{2}$ outside" layouts. The low flow rates used for the experiments, result in a laminar flow (Reynolds number is $\sim 280$ ). Obviously, this will result in much more tunnelling of liquid feed without interacting with the catalyst as compared to a packed bed geometry. Furthermore, the geometrical surface area between liquid and solid is lower for " $\mathrm{H}_{2}$ outside" and " $\mathrm{H}_{2}$ inside" than for "Packed bed". The $0.5 \mathrm{~mm}$ particles in the Packed bed layout create an external contact area one order of magnitude higher than the inner alumina wall of " $\mathrm{H}_{2}$ outside" and " $\mathrm{H}_{2}$ inside" layouts. " $\mathrm{H}_{2}$ inside" and "Packed bed" present the same high ammonia selectivity since both operate at the same high $\mathrm{H} / \mathrm{N}$ ratio. 


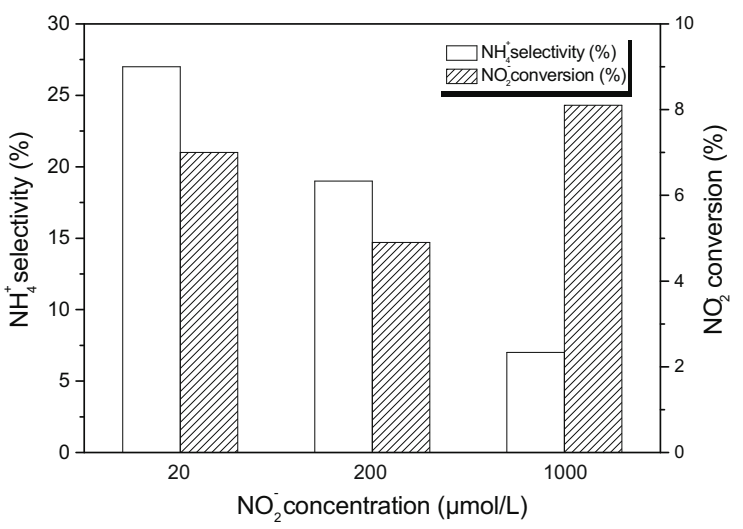

Figure 6.13: Nitrite conversion and ammonia selectivity of the " $\mathrm{H}_{2}$ outside" layout tested at different nitrite concentrations and constant hydrogen partial pressure $(0.2$ bar). Liquid flow rates were adjusted to obtain similar conversions as much as possible.

Table 6.2: Conversion, WHSV and reaction rate for the experiments shown in figure 6.11; note that the amount of $\mathrm{Pd}$ is identical in all three cases

\begin{tabular}{lllll}
\hline & $\mathrm{H}_{2}$ outside & $\mathrm{H}_{2}$ inside & Packed bed (1) & Packed bed (2) \\
\hline Conversion $(\%)$ & 4.8 & 4.1 & 4.2 & 24.9 \\
WHSV $\left(\mathrm{h}^{-1}\right)$ & 0.35 & 0.35 & 5.19 & 0.69 \\
Reaction rate $(\mathrm{mmol} / \mathrm{min})$ & $2.1 \times 10^{-6}$ & $1.8 \times 10^{-6}$ & $2.7 \times 10^{-5}$ & $2.2 \times 10^{-5}$ \\
\hline
\end{tabular}

\subsection{Conclusion}

A membrane reactor that allows low and homogeneous hydrogen concentrations in the axial direction has been successfully synthesized. It allows manipulation of the selectivity in the nitrite hydrogenation by establishing a low concentration of adsorbed hydrogen at the active sites. A comparison of different reactor layouts proved that this membrane reactor can be more selective towards nitrogen, the desired product, at identical conversion levels. This membrane reactor shows that it is possible to manipulate the selectivity towards nitrogen, the desired product. The low orders observed for both $\mathrm{H}_{2}$ and nitrite, combined with the strong effect of both concentrations on selectivity, provides evidence for a LangmuirHinshelwood mechanism with competitive adsorption of both reactants. 



\section{Bibliography}

[1] W. Ehrfeld, V. Hessel, and H. Löwe, Microreactors - new technology for modern chemistry (Wiley, 2001), No. 1, pp. 89-89.

[2] M. Vospernik, a. Pintar, G. Berčič, J. Batista, and J. Levec, Potentials of Ceramic Membranes as Catalytic Three-Phase Reactors, Chemical Engineering Research and Design 82, 659 (2004).

[3] S. Miachon and J.-a. Dalmon, Catalysis in membrane reactors : what about the catalyst?, Topics in Catalysis 29, 59 (2004).

[4] B. Gordon, P. Callan, and C. Vickers, WHO guidelines for drinking-water quality., WHO chronicle 38, 564 (2008).

[5] D. Shuai, J. K. Choe, J. R. Shapley, and C. J. Werth, Enhanced activity and selectivity of carbon nanofiber supported Pd catalysts for nitrite reduction., Environmental science \& technology 46, 2847 (2012).

[6] S. Hörold, T. Tacke, and K. Vorlop, Catalytical removal of nitrate and nitrite from drinking water: 1. Screening for hydrogenation catalysts and influence of reaction conditions on activity and selectivity, Environmental Technology 14, 931 (1993).

[7] M. H. Ward, T. M. DeKok, P. Levallois, J. Brender, G. Gulis, B. T. Nolan, and J. VanDerslice, Workgroup report: Drinking-water nitrate and health Recent findings and research needs, Environmental Health Perspectives 113, 1607 (2005).

[8] M. Shrimali and K. Singh, New methods of nitrate removal from water, Environmental Pollution 112, 351 (2001).

[9] K. Lüdtke, K.-V. Peinemann, V. Kasche, and R.-D. Behling, Nitrate removal of drinking water by means of catalytically active membranes, Journal of Membrane Science 151, 3 (1998).

[10] H. C. Aran, J. K. Chinthaginjala, R. Groote, T. Roelofs, L. Lefferts, M. 
Wessling, and R. G. H. Lammertink, Porous ceramic mesoreactors: A new approach for gas-liquid contacting in multiphase microreaction technology, Chemical Engineering Journal 169, 239 (2011).

[11] H. Aran, S. Pacheco Benito, M. Luiten-Olieman, S. Er, M. Wessling, L. Lefferts, N. Benes, and R. Lammertink, Carbon nanofibers in catalytic membrane microreactors, Journal of Membrane Science 381, 244 (2011).

[12] G. Strukul, R. Gavagnin, F. Pinna, E. Modaferri, S. Perathoner, G. Centi, M. Marella, and M. Tomaselli, Use of palladium based catalysts in the hydrogenation of nitrates in drinking water: from powders to membranes, Catalysis Today 55, 139 (2000).

[13] O. M. Ilinitch, F. Cuperus, L. V. Nosova, and E. N. Gribov, Catalytic membrane in reduction of aqueous nitrates: operational principles and catalytic performance, Catalysis Today 56, 137 (2000).

[14] S. M. Dutczak, M. W. J. Luiten-Olieman, H. J. Zwijnenberg, L. a. M. Bolhuis-Versteeg, L. Winnubst, M. a. Hempenius, N. E. Benes, M. Wessling, and D. Stamatialis, Composite capillary membrane for solvent resistant nanofiltration, Journal of Membrane Science 372, 182 (2011).

[15] S. Ebbesen, B. Mojet, and L. Lefferts, Effect of $p H$ on the Nitrite Hydrogenation Mechanism over $\mathrm{Pd} / \mathrm{Al}_{2} \mathrm{O}_{3}$ and Pt/Al2O3: Details Obtained with ATR-IR Spectroscopy, The Journal of Physical Chemistry C 115 (4), 1186 (2010).

[16] J. Chinthaginjala and L. Lefferts, Support effect on selectivity of nitrite reduction in water, Applied Catalysis B: Environmental 101, 144 (2010).

[17] Z. Zhao, G. Tong, and X. Tan, Nitrite removal from water by catalytic hydrogenation in a $\mathrm{Pd}$-CNTs- $\mathrm{Al}_{2} \mathrm{O}_{3}$ hollow fiber membrane reactor, Journal of Chemical Technology \& Biotechnology n/a (2015).

[18] R. Sander, Compilation of Henry's law constants, version 3.99, Atmos. Chem. Phys. Discuss. 14, 29615 (2014).

[19] N. a. Jarrah, J. G. van Ommen, and L. Lefferts, Growing a carbon nano-fiber layer on a monolith support; effect of nickel loading and growth conditions, Journal of Materials Chemistry 14, 1590 (2004).

[20] a. V. Melechko, V. I. Merkulov, T. E. McKnight, M. A. Guillorn, K. L. Klein, D. H. Lowndes, and M. L. Simpson, Vertically aligned carbon nanofibers 
and related structures: Controlled synthesis and directed assembly, Journal of Applied Physics 97, 041301 (2005).

[21] P.-G. de Gennes, F. Brochard-Wyart, and D. Quere, Capillarity and Wetting Phenomena (Springer-Verlag New York, 2004), pp. 128-129.

[22] T. R. Marrero and E. a. Mason, Gaseous Diffusion Coefficients, 1972.

[23] C. Franch, R. G. H. Lammertink, and L. Lefferts, Partially hydrophobized catalyst particles for aqueous nitrite hydrogenation, Applied Catalysis B: Environmental 156-157, 166 (2014).

[24] A. Pintar, J. Batista, and J. Levec, Potential of mono- and bimetallic catalysts for liquid-phase hydrogenation of aqueous nitrite solutions, Water Science and Technology 37, 177 (1998).

[25] I. Mikami, Y. Sakamoto, Y. Yoshinaga, and T. Okuhara, Kinetic and adsorption studies on the hydrogenation of nitrate and nitrite in water using $\mathrm{Pd}$-Cu on active carbon support, Applied Catalysis B: Environmental 44, 79 (2003).

[26] Y. Matatov-Meytal, Y. Shindler, and M. Sheintuch, Cloth catalysts in water denitrification III. $p H$ inhibition of nitrite hydrogenation over $\mathrm{Pd} / A C C$, Applied Catalysis B: Environmental 45, 127 (2003). 



\section{CHAPTER 7}

\section{Summary and Outlook}

\subsection{Summary}

Chapter 1 presents an overview on principles of photocatalysis (first part) and hydrogenation of nitrites (second part). Some important advanced oxidation processes (AOPs) are briefly explained. The general mechanism of photocacatalysis and different involving pathways are thoroughly explained. This is followed by an explanation of different configurations of photocatalytic reactors. The advantages and disadvantages of each are described. $\mathrm{TiO}_{2}$ as the most widely studied semiconductor photocatalyst is introduced and its properties are explained. Sputtering deposition and specifically reactive magnetron sputtering of $\mathrm{TiO}_{2}$ is introduced and explained. The favorable properties of microreactors for photocatalytic reactions are discussed and compared with other reactor configurations in terms of surface area to volume ratio. Kinetics of photcatalytic reactions are approached and the most frequently used methods for calculating photocatalytic reaction rates are mentioned. The second part concerns catalytic hydrogenation of nitrites. Membrane reactors and their advantages in heterogeneous catalysis are described. Different configurations of the membrane reactors are introduced and explained. Carbon nanofibers $(\mathrm{CNF})$ and their advantages as the catalyst support are described.

Chapter 2 presents a novel method for selective deposition of rutile and anatase $\mathrm{TiO}_{2}$ thin film. The oxygen content in the sputtering chamber is identified as the determining factor for deposition of either rutile or anatase $\mathrm{TiO}_{2}$. By monitoring the discharge voltage in DC reactive sputtering we detect a sharp transition from sub-stoichimetric to stoichiometric $\mathrm{TiO}_{2}$ as revealed by XPS analysis. The optical properties the $\mathrm{TiO}_{2}$ in either case is investigated. The 
sub-stoichiometric film absorbs in the visible range of the light spectrum while the stoichiometric is transparent in the visible range and absorbing in UV range. The dynamics of the annealing process is monitored by an in situ ellipsometry, showing the optical properties transformation from sub-stoichiometric to stoichiometric $\mathrm{TiO}_{2}$ thin film. The sub-stoichiometric and stoichiometric film after annealing are rutile and anatase as revealed by XRD analysis. Finally, the mobile carriers life time is investigated using electrodeless time-resolved microwave conductance technique (TRMC). The anatase $\mathrm{TiO}_{2}$ thin film displayed high photoconductance with longer lifetime charge carriers than rutile phase.

Chapter 3 describes the preparation of $\mathrm{TiO}_{2}$ (anatase) by DC reactive sputtering and its optical properties characterization. The prepared $\mathrm{TiO}_{2}$ is incorporated in a microreactor and the photocatalytic activity of the catalyst is evaluated for degradation of methylene blue (MB) in water. A numerical model which includes convection and diffusion for the flow channel combined with surface reaction is built and intrinsic reaction rate constants are extracted. Almost $90 \%$ degradation of MB is achieved within only 20 seconds residence time. The extracted surface reaction rate constant is also at least one order of magnitude higher compared to previous reports due to the enhanced mass transfer and uniform light distribution over the catalyst. Furthermore, by modeling the microreactor for different values of the second Damköhler number the regime where the rate of reaction is dependent on the chemical reaction or the mass transfer is determined. Finally the effect of $\mathrm{TiO}_{2}$ film thickness on the photocalytic performance is investigated. It is demonstrated that films thicker than $50 \mathrm{~nm}$ exhibit a constant photocatalytic activity.

In Chapter 4 we employed a state-of-the-art high resolution AFM to measure in situ, the forces between a silica probe and anatase $\mathrm{TiO}_{2}$ in an aqueous electrolyte solution. The effect of different substrates (p-Si, n-Si and p-Si/6 $\mu \mathrm{m}$ $\mathrm{SiO}_{2}$ ) on the surface properties of the $\mathrm{TiO}_{2}$ covering film is investigated. We probed in situ the local surface charge density at the patterned $\mathrm{TiO}_{2}$-electrolyte interface. We demonstrate that the surface charge density varies upon illumination, depending on the nature of the substrates supporting the thin $\mathrm{TiO}_{2}$ films. The corresponding photocatalytic activities are evaluated using a microreactor and intrinsic surface averaged reaction rate constants are extracted using a model 
which includes convection and diffusion inside the microchannel. We observed that the highest photocatalytic performance belongs to the $\mathrm{p}-\mathrm{Si} / \mathrm{TiO}_{2}$ due to the enhanced charge separation which is in a good agreement with AFM studies.

Chapter $\mathbf{5}$ describes the fabrication and modeling of an immobilized porous $\mathrm{TiO}_{2}$ thin film microreactor. Optical properties of the prepared porous $\mathrm{TiO}_{2}$ are investigated using spectroscopic ellipsometry and compared with transmission measurements. A numerical model is built for both light independent and light dependent reaction kinetics. In addition the effect of light is considered by defining a criterion for neglecting light intensity based on the porous $\mathrm{TiO}_{2}$ film thickness. The chapter concludes by presenting a performance parameters based on the internal effectiveness factor.

Chapter 6 demonstrates the utilization of a porous alumina hollow fiber with impregnated carbon nanofibers (CNFs) as catalyst supports for catalytic hydrogenation of nitrites. CNFs growth parameters are optimized in terms of surface area and quality of the resulted CNFs. The selectivity towards nitrogen is investigated by comparing the alumina hollow fiber reactor, where the hydrogen and nitrite in liquid are fed separately, with different reactor configurations. The membrane reactor realizes a low and homogeneous hydrogen concentration over the axial direction of the reactor allowing selectivity modification by limiting the presence of adsorbed hydrogen on the catalyst active sites. As a result the membrane reactor is 2-3 times more selective towards nitrogen compared to the other studied reactor configurations.

\subsection{Outlook}

\subsubsection{Scaling up; Design and fabrication of a disk reactor}

As described in the previous chapters, photocatalytic reactions in microreactors are significantly faster compared to other reactor configurations due to enhanced mass and photon transfer. However due to their small volume the throughput is significantly lower than the conventional reactors and long residence times are required. Degradation of some organic compounds, would only be possible at very low flow rates. Therefore we designed and fabricated a disk microreactor 
possessing a larger volume while keeping a low channel height $(100 \mu \mathrm{m})$. The disk microreactor consists of two parts (figure 7.1); a borofloat glass wafer as the channel compartment and a silicon wafer (p-type (100)) as the $\mathrm{TiO}_{2}$ thin film support.

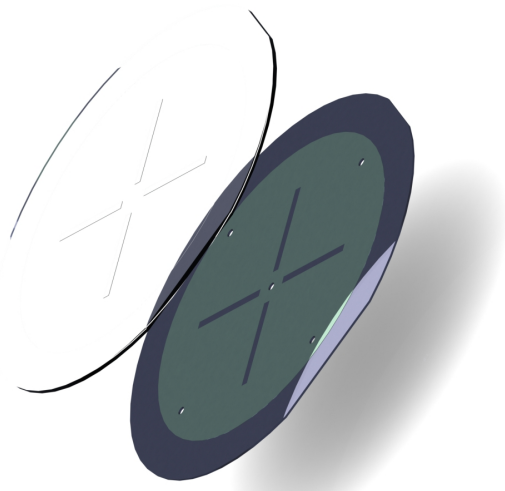

Figure 7.1: Graphical illustration of the disk microreactor consisting of glass and silicon wafers.

Figure 7.2 (right) shows the degradation of methylene blue as a function of different flow rates. As observed methylene blue is degraded almost $70 \%$ at 2 $\mathrm{ml} / \mathrm{min}$ flow rate. This flow rate is at least 200 times faster than the required flow rate for the same degradation level in the PDMS based microreactor explained in chapter 2.
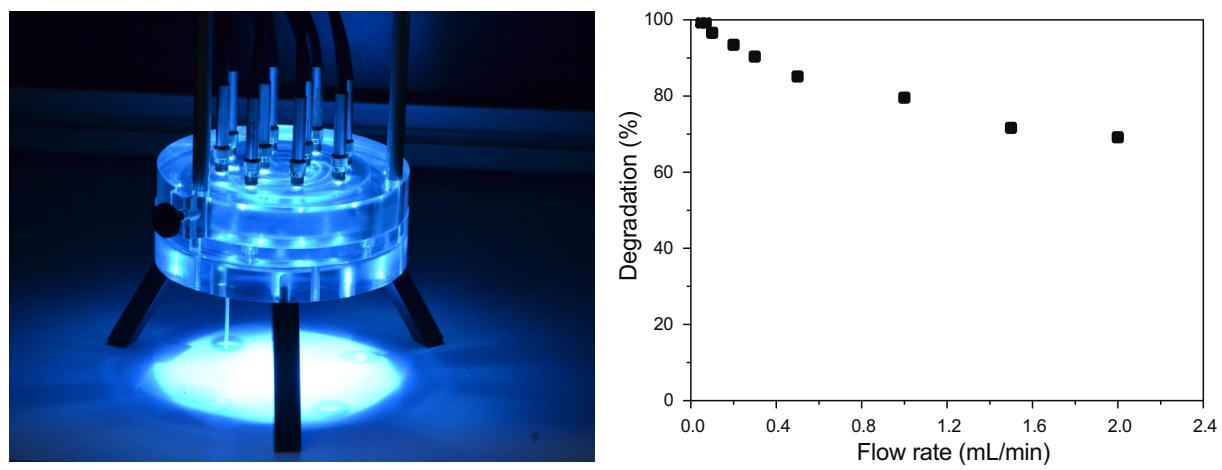

Figure 7.2: Operation of the disk microreactor (left) and degradation of methylene blue as a function of flow rate (right). 


\subsubsection{Catalytic hydrogenation of nitrate combined with photocatalysis}

Nitrate hydrogenation reaction is performed on a bimetallic catalyst. The bimetallic catalyst consists of a noble metal $(\mathrm{Pt}, \mathrm{Pd}, \mathrm{Ru})$ and a promoter metal $(\mathrm{Cu}$, $\mathrm{Sn}, \mathrm{In}, \mathrm{Ag})$. In the reaction firstly nitrate is reduced to nitrite on the bimetallic catalyst and secondly nitrite is reduced to nitrogen and ammonia on the noble metal (monometallic catalyst) as described in chapter 6. This reaction has often been performed in different reactor configurations such as monolith, slurry and membrane reactors in which hydrogen is dissolved in the liquid and is fed with the nitrite stream. Recently, the use of membrane reactors for nitrate/nitrite hydrogenation has been investigated. One of the advantages is that liquid and gas streams are supplied from different sides of the membrane and therefore allowing an independent control of both gas and liquid phases. As shown in chapter 6, $\mathrm{H} / \mathrm{N}$ ratio on the catalyst surface has a determining factor on the selectivity of nitrite hydrogenation reaction. Low $\mathrm{H} / \mathrm{N}$ ratios results in high nitrogen selectivities. Therefore, by using a membrane reactor, low and homogeneous hydrogen concentrations can be fed to the system, causing high nitrogen selectivity (low $\mathrm{H} / \mathrm{N}$ ratios). Moreover another disadvantage of the conventional reactors where hydrogen dissolved in liquid is that, low hydrogen concentrations cannot be fed, otherwise, depletion of hydrogen would cause low conversions and low reaction rates. For instance in case of packed bed reactors most of the fed hydrogen is consumed at the fist part of the reactor leaving the rest of the reactor inefficient. An intriguing approach is using organic reducing agents like formic acid for hydrogen generation instead of direct hydrogen feeding. Formic acid for instance gradually decomposes on noble metal particles ( $\mathrm{Pd}$ or $\mathrm{Pt}$ ) giving hydrogen required for the nitrate and nitrite hydrogenation to nitrogen and ammonia. The advantage is that hydrogen concentration can be tuned by controlling the decomposition of formic acid via a good control of the temperature and the formic acid concentration in the system. However, formic acid decomposes generally at temperature higher than $50 \mathrm{C}$ [1] in liquid phase which can have adverse effects on the efficiency of the process.

Chenbiao et al. [2] reported on the formation of molecular hydrogen from pho- 


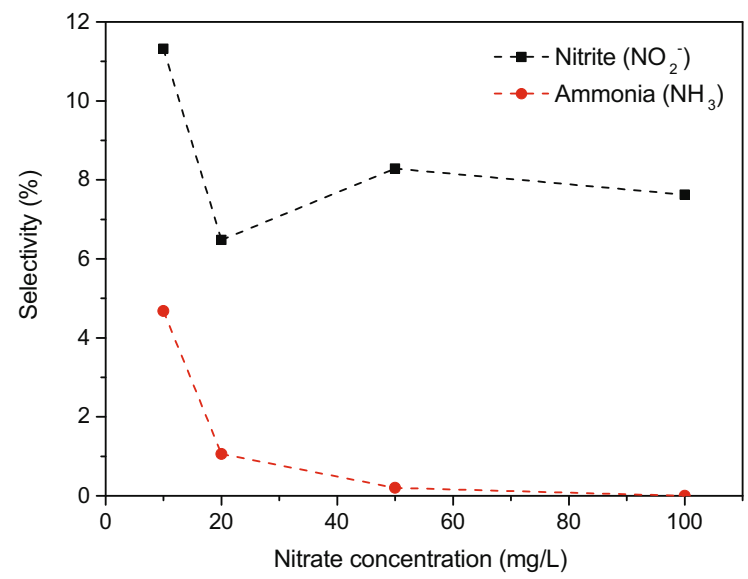

Figure 7.3: Selectivity of ammonia and nitrite as a function of nitrate concentration.

tocatalytic decomposition of methanol on titanium dioxide. Based on this idea we deposited $\mathrm{Cu} / \mathrm{Pd}$ (bimetallic catalyst) on the porous $\mathrm{TiO}_{2}$ (chapter 5 ) and incorporated it in the microreactor described in chapter 3,4,5. The microreactor is fed with the mixture of nitrate and methanol. The required hydrogen for nitrate reduction was supplied in-situ by photocatalytic decomposition of methanol on $\mathrm{TiO}_{2}$. The preliminary results were promising in terms of nitrogen selectivity. As shown in figure 7.3 by increasing the nitrate concentration the nitrite selectivity slightly decreases and becomes stable while ammonia selectivity decreases reaching to almost zero at $100 \mathrm{mg} / \mathrm{L}$ nitrate concentration. 


\section{Bibliography}

[1] D. A. Bulushev, S. Beloshapkin, and J. R. Ross, Hydrogen from formic acid decomposition over Pd and Au catalysts, Catalysis Today 154, 7 (2010).

[2] C. Xu, W. Yang, Q. Guo, D. Dai, M. Chen, and X. Yang, Molecular Hydrogen Formation from Photocatalysis of Methanol on anatase- $\mathrm{TiO}_{2}$ (110), Journal of American Chemical Society 136, 602 (2014). 



\section{Algemene Nederlandse samenvatting}

Hoofdstuk 1 geeft een overzicht van de principes van fotokatalyse (eerste deel) en de hydrogenering van nitrieten (tweede deel). In het eerste deel worden enkele belangrijke geavanceerde oxidatieprocessen (AOPs) kort toegelicht. Het algemene mechanisme van fotokatalyse en verschillende relevante routes worden in detail uitgelegd. Vervolgens wordt een uitleg gegeven van verschillende configuraties van fotokatalytische reactoren, met een beschrijving van de voor-en nadelen van elke configuratie. Titaniumdioxide $\left(\mathrm{TiO}_{2}\right)$, de meest gebruikte halfgeleidende fotokatalysator, wordt gentroduceerd en de eigenschappen van dit materiaal worden uitgelegd. Depositie middels sputteren en in het bijzonder reactive magnetron sputtering van $\mathrm{TiO}_{2}$, wordt geintroduceerd en toegelicht. De gunstige eigenschappen van microreactoren voor fotokatalytische reacties worden besproken en vergeleken met andere reactorconfiguraties in termen van de verhouding van oppervlakte tot volume. De kinetiek van fotokatalytische reacties wordt kort behandeld en de meestgebruikte methoden voor het berekenen van fotokatalytische reactiesnelheden worden benoemd. Het tweede deel betreft de katalytische hydrogenering van nitrieten. Membraanreactoren en hun voordelen in heterogene katalyse worden beschreven. Verschillende configuraties van membraanreactoren worden gentroduceerd en uitgelegd. Tevens worden koolstof nanovezels beschreven en wordt hun geschiktheid als drager voor katalysatoren behandeld.

Hoofdstuk 2 presenteert een nieuwe methode voor de selectieve depositie van dunne filmen van rutiel en anataas $\mathrm{TiO}_{2}$. Het zuurstofgehalte in de sputterkamer werd geidentificeerd als de bepalende factor voor de depositie van rutiel dan wel anataas $\mathrm{TiO}_{2}$. Door het monitoren van de ontladingsspanning in DC reactief sputteren hebben wij, zoals bleek uit XPS-analyse, een scherpe overgang van sub-stoichiometrisch naar stoichiometrisch TiO2 gedetecteerd. De op- 
tische eigenschappen van $\mathrm{TiO} 2$ voor beide gevallen waren onderzocht. De substoichiometrische film absorbeert licht in het zichtbare gebied van het spectrum, terwijl de stoichiometrische film transparant is in het zichtbare gebied en licht in het UV-gebied absorbeert. De dynamische eigenschappen van het annealing proces werden gemonitord middels in-situ ellipsometrie, waarmee de transformatie van de optische eigenschappen van sub-stoichiometrische naar stoichiometrische dunne $\mathrm{TiO}_{2}$ filmen zichtbaar werd. De sub-stoichiometrische en stoichiometrische film na annealing zijn rutiel en anataas, respectievelijk, zoals aangetoond met een XRD-analyse. Tot slot werd de levensduur van mobiele ladingsdragers onderzocht door gebruik te maken van de electrodeless time-resolved microwave conductance (TRMC) techniek. In vergelijking met rutiel $\mathrm{TiO}_{2}$ vertoonden dunne filmen van anataas $\mathrm{TiO}_{2}$ hogere fotogeleiding door ladingsdragers met een langere levensduur.

Hoofdstuk 3 beschrijft de bereiding van $\mathrm{TiO}_{2}$ (anataas) met DC reactief sputteren en de karakterisering van zijn optische eigenschappen. De gemaakte $\mathrm{TiO}_{2}$ is geintegreerd in een microreactor, en de fotokatalytische activiteit van de katalysator werd vastgesteld voor de degradatie van methyleenblauw (MB) in water. Er is een numeriek model opgesteld waarin convectie en diffusie voor het stromingskanaal wordt gekoppeld aan een oppervlaktereactie, en waarmee intrinsieke reactiesnelheden werden bepaald. Bijna $90 \%$ degradatie van MB werd behaald bij slechts 20 seconden verblijftijd. De bepaalde oppervlaktereactieconstante is tevens minstens $\mathrm{n}$ ordegrootte groter dan eerder gerapporteerde constanten dankzij het versnelde massatransport en de uniforme verdeling van licht over de katalysator. Verder werd, door het modelleren van de microreactor voor verschillende waarden van het tweede Damkhlergetal, het regime bepaald waarvoor de reactiesnelheid afhankelijk is van de chemische reactie of de massaoverdracht. Tot slot werd het effect van de dikte van $\mathrm{de}^{\mathrm{TiO}}{ }_{2}$ film op de fotokatalytische prestaties onderzocht. Het bleek dat voor een filmdikte groter dan $50 \mathrm{~nm}$ de fotokatalytische activiteit constant is.

Hoofdstuk 4 beschrijven wij het gebruik van een state-of-the-art hoge res0lutie AFM om in situ de krachten tussen een silica punt en anataas $\mathrm{TiO}_{2}$ in een waterige elektrolytoplossing te meten. Het effect van verschillende substraten (p$\mathrm{Si}$, n-Si en p-Si/6 m $\mathrm{SiO}_{2}$ ) op de oppervlakte-eigenschappen van de $\mathrm{TiO}_{2}$ film 
op het substraat werd onderzocht. We maten in situ de lokale dichtheid van de oppervlaktespanning op het gestructureerde grensvlak tussen $\mathrm{TiO}_{2}$ en het elektrolyt. Wij toonden aan dat de ladingsdichtheid van het oppervlak afhangt van de belichting, afhankelijk van de eigenschappen van het substraat onder de dunne $\mathrm{TiO}_{2}$-filmen. De bijbehorende fotokatalytische eigenschappen werden bepaald met behulp van een microreactor, en de intrinsieke reactieconstanten werden berekend met een model dat rekening houdt met convectie en diffusie in de microkanalen. Wij vonden dat $\mathrm{p}-\mathrm{Si} / \mathrm{TiO}_{2}$ de beste fotokatalytische prestaties vertoonde vanwege de verbeterde ladingsdeling, zoals in overeenstemming met de AFM-metingen.

Hoofdstuk 5 beschrijft de fabricage en de modellering van een gemmobiliseerde poreuze dunne $\mathrm{TiO}_{2}$-film reactor. De optische eigenschappen van de bereide poreuze $\mathrm{TiO}_{2}$ werd onderzocht middels spectroscopische ellipsometrie en vergeleken met lichttransmissiemetingen. Een numeriek model was opgesteld voor zowel lichtonafhankelijke als lichtafhankelijke reactiekinetiek. Daarnaast wordt het effect van licht bekeken door een criterium op te stellen, gebaseerd op de dikte van de poreuze $\mathrm{TiO}_{2}$-film, voor het negeren van de lichtintensiteit. Het hoofdstuk besluit met het geven van een prestatieparameter gebaseerd op de interne effectiviteitsfactor.

Hoofdstuk 6 laat het gebruik zien van poreuze alumina holle vezels gempregneerd met koofstof nanovezels (carbon nanofibers, CNFs) als drager van katalysatoren voor de katalytische hydrogenering van nitrieten. De parameters voor de groei van CNFs zijn geoptimaliseerd in termen van oppervlakte en kwaliteit van de resulterende CNFs. De selectiviteit voor stikstof was onderzocht door de alumina holle vezelreactor, met een separate voeding van waterstof en van in water opgelost nitriet, te vergelijken met verschillende andere reactorconfiguraties. Met de membraanreactor kon een lage en homogene waterstofconcentratie over de axiale richting van de reactor gerealiseerd worden. Dit staat het toe om de selectiviteit te reguleren door de aanwezigheid van geadsorbeerd waterstof op de actieve centrum van de katalysator te limiteren. Daardoor is, in vergelijking met de andere bestudeerde reactorconfiguraties, de membraanreactor twee- tot driemaal selectiever voor stikstof. 



\section{Acknowledgments}

My phd tree after almost four years of taking care, trimming and irrigation bears fruits in winter:). This would not be possible without the help of many people that in this part I want to acknowledge them and bring a smile to their face. I will do my best to recall all the people but if I won't be successful blame it on my memory (every one knows I have a great memory).

First of all I would like to express my gratitude to my daily supervisor Prof. Dr. Rob Lammertink for giving me the opportunity to pursue my phd in his group. You motivated me whenever I was disappointed and lit my way whenever I was in dark. You smartly advised me how I have to allocate my time to different projects. I learned from you that the key to success is looking always one step forward. I appreciate that your office was always open and you answer my questions patiently, even at the time you had a very tight schedule. I am always amazed by your ability to handle different projects so fast and efficiently without sacrificing the quality. Thank you for reviewing my thesis and your suggestions. And most importantly thank you for being a very nice friend for me; a person who I could get not only scientific advice. I never forget your and Cindy's hospitality in Stanford we visited you. I feel really happy and lucky have worked with you in the past years. I wish a prosperous life for you and your family and hearing many awesome things from SFI.

I would like to thank Prof. Guido Mul for reading my thesis, accepting to be my committee member and also for the useful discussions. Many thanks go to Prof. Leon Lefferts for reading my thesis, accepting to be my committee member and advice on hydrogenation of nitrite project (chapter 6). Appreciation also goes out to Dr. Tom Savenije for reading my thesis, accepting to be my committee member. I would like to also thank you for your comments on chapter 2 and for the TRMS measurements on my samples which altogether lead to a nice 
publication (chapter 2). I would like to thank Prof. Han Gardeniers for reading my thesis, accepting to be my committee member and the useful feedback during the SFI-MCS meetings. I would like to acknowledge Prof. Kristof Demeestere for reading my thesis and accepting to be my committee member.

I would like to thank Dr.Ir. Herbert Wormeester for the useful advice on my ellipsometry work which were implemented in chapter 2. I also appreciate the help of Wojciech Ogieglo for the ellipsometry measurements and all fruitful discussions we had. I would like to express my gratitude to Prof. Frieder Mugele for the feedback for our collaboration on the AFM measurements of titania (chapter 4). Many thanks also go to Dr. Igor Siretanu for all the AFM measurements and calculations of the surface charges on the patterned $\mathrm{TiO}_{2}$. I also want to thank you for the last optimization of chapter 4 of my thesis, through the many interesting discussions we had. I would not be able to finish my Phd without the technical support of the following people. I would like to thank Ineke Punt, the technician of our group, whom I got the first instructions on working in the lab, PDMS preparation, making different set-ups and working with SEM. I always enjoy to get instructions from you due to your calm and relaxing character. I would like to thank Stephan and Roald for the beneficial guidelines on the fabrication of microreactors in cleanroom. I would like to thank Jan van Nieuwkasteele for the help in the cleanroom and your useful advice on microfabrication. I would like to show my gratitude to Mark Smithers for the nice and clear HR-SEM micrographs which I used throughout of my thesis. I enjoyed also talking to you about different topics in the SEM room. I would like to thank other cleanroom staff: Gerard, Christiaan, Ton, Robert, Rene, Huib, Hans and Samantha for your kind help. I would like to thank Bert for your friendly personality and designing the module for Alumina Hollow Fiber reactor (chapter 6). Many thanks go to Lidy, our secretary, for being always helpful and responsive even on holidays. John Heeks, I will be ever grateful for your kind support during the first year of my Phd. Your memory will always be with me. I wish you were here and I could share my happiness with you:( It is a pleasure to thank my students, Rick, Tom, Aura and Justin. Thank you for your contributions on this work.

It is time to acknowledge my family; First of all I owe my deepest gratitude to my grandfather Dr. Mohammad Kamali who was always inspiring for me. I 
remember when I was a child you were invited to give talks in different conferences worldwide and I deeply wished one day I could be like you. You were, are and will be my role model in my whole life. I never forget you were always telling me for being a good researcher I should be like a flowing river in the bank, hitting the stones and obstacles on the way but continue flowing. It grieves me to say how many things I planned to do with you when you come here for my graduation ceremony but sadly nature did not allow it... You are always with me and time will not make your memories fade away. My mother, Shideh, my father, Tahmoores, my sister Dorreh and my grandmother Afsar thank you so much for your many years of supports, encouragements and positive energy during my studies. I am proud to have you in my life.

Now it is time to acknowledge my friends and colleagues for sharing my happiness. Can, you are one of the amazing persons I met in my life. I remember you were my first close friend in Netherlands. You helped me a lot in my project and life in Netherlands. We have lots of nice memories that whenever I remember it makes me laugh to death. I could never forget the sunny boulevard and winter corner serious discussions we had. Thank you for sleeping in the first minutes of the movies that I strongly and passionately recommended you to watch. Thank you for allowing Erik and I to discover your hidden talent in singing and naming our band "persian carpet" due to my characteristics. I hope we can release our album finally since many people are crazy to hear it. Many many things if I want to write it is going to be three thesis..... I wish all the best for you, Laura and lovely Nora and I hope we see each other more often. Erik thank you so much for setting new appointments in my agenda while I was not in the office. That was really kind of you. I remember one time one of your events popped up while I had a very important meeting. Your enthusiasm for taking me to gym is highly appreciated but I think you were more successful with Krzyskof. I can never forget our memories in the micronano conferences and different courses. Thank you for ordering food at the hotel at 3 am and going to sleep. Sinem thank you for being always supportive and to defend on the same day as me. We can share our stress together and reduce it in this way. Olga thank you for selecting me as the best Olga. One day you will be the second best director (First is Korean director Kim-Ki-Duk) if your multiple holidays allow. I think it is time to holi- 
day on a new planet since you traveled to everywhere in this planet. Yali, you are the most amazing office-mate that I could ever have. We shared lots of ups and downs during our Phd but always after talking together everything became smooth. We started exactly on the same day, we submitted our thesis on the same day and we are going to defend exactly on the same day. Thank you and Jie for making tasty hotpots every now and then at your place. I really like it. Sander thank you for helping me on writing the Nederlandse Samenvatting. You did a great job. Thank you also that sometimes you provide chocolates, do it more often. I enjoy talking to you about science, life and everything. I wish you have a very happy life together with Corine, your lovely daughter Marieke and the upcoming member of your family. Jeff, my amazing hilarious encyclopedia friend. I am always intrigued by your general knowledge. You almost have answer to any questions you are ever asked. I enjoyed a lot chatting with you and learning many things from you. I wish you full of success in your new career. By the way I like your facial expressions when you are reading these lines. Aura my former student, current Phd student and my always friend. We had lots of happy and sad moments working together during your master project but finally it led to a very nice publication that we both are proud of. I never forget the day you dissolve my mold in acid and we were looking each other without saying anything for a while:) Anne thank you for explaining so enthusiastically the genealogy of the animals with special emphasis on rabbit and bunny:) Khalid thank you for being always calmly answer my questions about Arabic language. Believe me I know a thing or two but on grammar. Joeri thank you for explaining your PhD project when we are super drunk:) Hanieh, I am super happy that you will start your PhD in the group. I hope everything goes as planned for you and you enjoy your stay in Netherlands. Krzysztof thank you for being sometimes very lame. Your lameness provokes our creativity to compose an amazing song that nowadays people sing it everywhere. Thank you also for organizing professional polish dance competitions. Roger (Rogina) you are my brother here in Netherlands this means a lot to me. Thank you for helping me discovering new places at university that we both benefited from it:) I hope we can finalize our dictionary "Badman" like Longman as soon as possible. Just give you an example; Try not to be cinema and practice thunders. Avoid going to highways without insurance. 
Don't do investment when the beast is awake and many more "Pesaram". Maria thank you for your warm hospitality while I was alone in Enschede. Thank you also for dealing with Roger's multiple hangovers:) I hope this year finally we could arrange our long-awaited Japanese trip. I am also enthusiastically waiting to hug the upcoming member of your family. Milad thank you so much for all the nice conversation during the coffee breaks. I am honored to give you the medal of alcohol tolerance as revealed in my first year Disco Damon parties. Hossein (Wasabi) thank you for being so crazy and being roommate with Milad, I know it is difficult. Mojtaba and Taher, we had lots of sad and happy moments during our phd and sharing them together always makes the bads less bad and goods better Berim Asatid?. Amir, Shahrzad, Aidin, Maral, Kasra and Nasim thank you for your intimate friendship and all the fun we have had and we will have of course.

Last but not definitely least I would like to thank my love, wife, friend, companion and simply everything, Saghar. I am sure without you I could not definitely reach to this point. You have been always my inspiration and motivation for continuing my education. Whenever I am disappointed your magic words make the world beautiful for me again. You can not believe how much I become mesmerized whenever you shine like a diamond on stage. You rock...

- Damon 

"Maybe you are searching among the branches, for what only appears in the roots"

Rumi

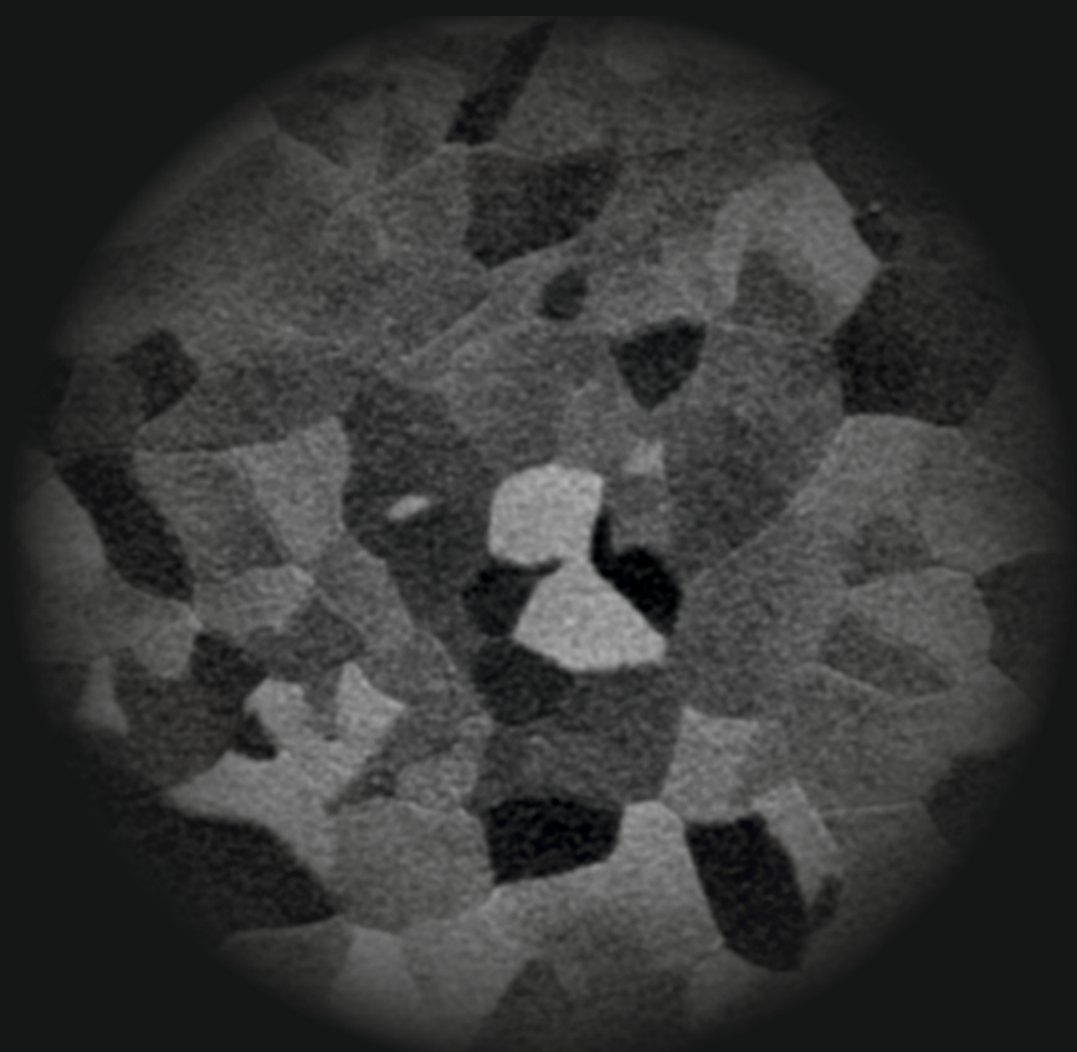

ISBN: 978-90-365-4078-0 